\title{
Human pharmacology of tranquilizing drugs
}

\begin{abstract}
The widespread confusion in the classification of various tranquilizing drugs is due to the fact that there are no specific tranquilizers at this time. All available drugs have numerous pharmacologic actions in addition to ataraxia. Some of the newer drugs used to relieve anxiety are related pharmacologically to the well-known classic sedative and soporific compounds. Some agents, like chlorpromazine and reserpine, etc., have much less soporific effect but do have other marked pharmacologic actions. The position of the reviewer is that there are no truly specific tranquilizers and that most central nervous system depressants in proper dose or circumstance may be used clinically in man to relieve anxiety and produce tranquilization. The concept of the dose-response relationship of these agents is discussed. Drugs with relatively flat dose-response curves for central nervous system depression tend to be the most useful in relieving anxiety without producing too many undesirable actions.

The pharmacology of several selected agents and related congeners is discussed in detail. These include the substituted phenothiazines, the Rauwolfa alkaloids, meprobamate, and chlordiazepoxide.
\end{abstract}

Edward F. Domino, M.D. Ann Arbor, Mich. Department of Pharmacology, University of Michigan

It is easy to define a tranquilizer as a substance which relieves anxiety and produces calmness, peacefulness, serenity, placidity, or ataraxia. It is not easy, however, to select specific pharmacologic agents to include in a rational classification of tranquilizers. Numerous central nervous system depressants, some stimulants, and a few drugs with no central effects whatsoever may in proper dose and circumstance relieve anxiety and produce tranquilization. Few physicians would object to the classification of reserpine, chlorpromazine, and their related congeners as tranquilizing

Received for publication April 2, 1962. agents. There would be considerable disagreement, particularly among psychiatrists and pharmacologists, as to the precise categorization of ethyl alcohol, barbiturates, meprobanate, hydroxyzine, benactyzine, chlordiazepoxide, and other nonbarbiturate sedatives.

It is usually assumed that tranquilizers induce psychologic and physiologic states qualitatively different from sedative-soporific drugs. Few would disagree with the marked differences in pharmacology between chlorpromazine and the barbiturates, but many would disagree with claims made that meprobamate is pharmacologically uniquely different from some barbiturates 
or ethyl alcohol. Inasmuch as there are inadequate data on the uniqueness of the psychologic states induced by these agents, it is readily understandable why disagreement and confusion are widespread. This is reflected and propounded by the myriad of advertisements referring to numerous heterogeneous drugs as calmative, eunoic, relaxant, neurosedative, psychosedative, or even more imaginative terms. ${ }^{278}$

Numerous classifications of tranquilizing drugs have been suggested. Berger ${ }^{28}$ proposed a classification based in part on chemical structure and in part on the presence or absence of autonomic nervous system-blocking properties. Reserpine, the substituted phenothiazines, and related compounds were considered central autonomic suppressants. On the other hand, propanediols such as meprobamate and various diphenylmethane derivatives were classified as tranquilizers without autonomic-blocking properties. Such a proposal did little to define the place of barbiturates. Pfeiffer and colleagues ${ }^{248}$ provided evidence that meprobamate has some pharmacologic properties similar to barbiturates, including the production of "barbiturate-like" fast waves in the electroencephalogram of man. Are other sedative drugs to be classified along with meprobamate as tranquilizers without autonomic-blocking properties?

Ross and Cole $^{268}$ proposed a classification based on psychiatric usage in which agents were classified as major or minor tranquilizing drugs. Schiele and Benson ${ }^{273}$ have suggested a similar classification. They defined major tranquilizing drugs as those characterized by (1) production of emotional calmness and mental relaxation, (2) high effectiveness in controlling the symptoms of acutely and chronically disturbed psychotic patients, (3) production in susceptible patients of reversible extrapyramidal symptoms, and (4) little to no tendency to produce physical dependence or habituation. These agents are drugs of choice in excited, delusional, and psychotic states. The minor tranquilizing drugs were characterized as agents which produced calmness and re- laxation but not of the same "quality" as the major tranquilizers. Precisely what was meant by this was difficult to define. The authors suggested that such agents are particularly useful in common psychoneurotic problems but are not very effective in treatment of severely disturbed psychotic patients. These agents do not produce extrapyramidal symptoms and have a relatively low incidence of annoying effects, except that physical dependence does occur to some. The minor tranquilizers are preferred in common nervous states such as nervous tension, mild depression, psychosomatic disorders, etc.

Seevers $^{278}$ has suggested that because of the marked heterogeneity of pharmacologic substances called tranquilizers and because knowledge of the primary sites of actions of these compounds is extremely limited, the term tranquilizer be abandoned as having any specific pharmacologic meaning. He proposed that such drugs be classified on the basis of their predominant pharmacologic actions in man based upon whether the agents produced nonselective or selective depression of the central nervous system. There is ample evidence that Seevers is fundamentally correct in his aversion to "tranquilizer" as having no specific pharmacologic connotation. Friend, ${ }^{103}$ in his review of tranquilizers, includes reserpine and its derivatives, phenothiazines, propanediols, diphenylmethanes, barbiturates, and a host of nonbarbiturate sedatives. In the same publication, Pelikan and Kensler ${ }^{244}$ review these agents as sedatives. The latter have reviewed the problem of defining sedation. They suggest that clinically the sedative properties of reserpine and chlorpromazine seem qualitatively different from those of the barbiturates and other related compounds. Certainly, animal as well as human toxicity data would support the notion that deep coma or anesthesia is usually not seen after large doses of reserpine and chlorpromazine as compared to the barbiturates. Electroencephalographic and behavioral arousal may occur with increased afferent input in both ani- 
mals and man after large doses of reserpine and chlorpromazine but not after large doses of barbiturates. Evidence in animals and man on the sites of action of these and other central nervous system depressants clearly indicates that there are remarkable qualitative differences. ${ }^{83}$ Nevertheless, it is of considerable interest that Klerman and colleagues $^{173}$ could not distinguish between the qualitative effects of three psychoactive drugs given in therapeutic doses to normal human subjects. These investigators compared on a double blind basis two dose levels of meprobamate (as a tranquilizer), phenyltoloxamine (as a sedative-antihistaminic), and secobarbital (as a sedativehypnotic) and a placebo given in single oral doses. The effects of these agents were observed on (1) various physiologic functions including heart rate, respiration, blood pressure, skin temperature, muscle tension, and pupil size, (2) level of consciousness and alertness as determined by rating scores, (3) psychomotor performance as measured by tapping speed and pursuit rotor coordination, (4) mental functioning as measured by serial addition and a learning task, (5) subjective activity, and (6) behavior during a psychiatric interview. The data obtained indicated that the three psychopharmacologic drugs used had somewhat similar effects. On the basis of these results as well as those obtained from a previous study with phenyltoloxamine and reserpine, ${ }^{79}$ Klerman and colleagues concluded that the special properties of "tranquilizing" drugs do not lie in their ability to induce psychologic states uniquely different from sedation. Rather, the critical feature was related to the dose margins between sedative effects and those affecting psychomotor performance and consciousness. Thus, they considered sedation and tranquilization as similar psychologic states. Clinically, an important difference between a tranquilizer and a sedative is the greater degree of soporific effect produced by the latter.

From a pharmacologic point of view, the list of agents used clinically as tranquilizers includes drugs with many diverse actions. Numerous agents have been found empirically in man to reduce the intensity of hyperemotional states such as anxiety. At the present time, there are no drugs which have only this specific property. To date, drugs which depress anxiety have numerous other actions in the body. For example, narcotics such as morphine are effective in relieving anxiety but have marked addiction liability which prevents their widespread use as tranquilizers. Many of the sedative-hypnotics such as phenobarbital likewise reduce anxiety but may in larger dosage cause marked drowsiness. The newer tranquilizing drugs are said to have fewer adverse effects but do in fact have many diverse pharmacologic actions. At the present time, there are no specific tranquilizing drugs.

Perhaps what determines whether a sedative drug is used clinically to produce tranquilization is the slope of its dose-response curve for central nervous system depression. A drug with a shallow dose-response curve probably will be a useful tranquilizing agent because there will be a greater spread between the dose producing weak sedation (or tranquilization) and the dose producing a soporific effect. Although qualitative differences exist between sedativehypnotics (like barbiturates) and some tranquilizers (like reserpine and chlorpromazine), one must be aware that in proper dose and circumstance, a variety of central nervous system depressants may produce the clinical phenomenon of tranquilization. It is, therefore, my view that most central nervous system depressants can be classified as tranquilizing drugs.

The following classification is proposed of some central nervous system depressants which in proper dose or circumstance may produce tranquilization:

I. Drugs with primarily sedative-soporific effects A. Bromides

B. Alcohols

1. Ethanol

2. Chloral hydrate

3. Petrichloral 
C. Aldehydes

1. Paraldehyde

D. Acetylenic carbinols and derivatives

1. Methylparafynol

2. Ethchlorvynol

3. Ethinamate

E. Urea derivatives

1. Monoureids

a. Carbromal

b. Bromisovalum

c. Ectylurea

2. Diureids

a. Barbiturates

F. Piperidinediones

1. Methyprylon

2. Glutethimide

G. Drugs of miscellaneous chemical structure with alleged skeletal muscle relaxant, analgesic, or other more selective central actions

I. Meprobamate

2. Promoxolane

3. Phenaglycodol

4. Chlormethazanone

5. Carisoprodol

6. Chlordiazepoxide

7. Phenyramidol

8. Mephenoxalone

9. Mebutamate

10. Hydroxyphenamate

II. Narcotic analgesics, including morphine and similar agents

III. Drugs with predominantly antihistaminic effects

1. Diphenhydramine

2. Hydroxyzine

3. Promethazine

4. Phenyltoloxamine

5. Chlorphenoxamine

IV. Drugs with predominantly catecholamine and serotonin depleting effects

A. Rauwolfia alkaloids

1. Reserpine

2. Alseroxylon

3. Deserpidine

4. Rescinnamine

5. Syrosingopine

B. Synthetic agents

1. Tetrabenazine

2. $\alpha$-Methyl DOPA

V. Substituted phenothiazines with predominantly catecholamine and serotonin blocking effects

1. Promazine

2. Chlorpromazine

3. Thioridazine

4. Mepazine

5. Proclorperazine

6. Perphenazine

7. Thiopropazate

8. Triflupromazine
9. Trifluoperazine

10. Fluphenazine

VI. Drugs with predominantly cholinergic blocking effects

A. Some Solanaceae alkaloids such as scopolamine

B. Benactyzine

Drugs such as ethyl alcohol or the barbiturates produce various degrees of central nervous system depression, depending upon dose. Relatively small doses of these agents produce a mild depression of the central nervous system which may be considered weak sedation or tranquilization in man. With pentobarbital, this might occur with oral doses of perhaps $50 \mathrm{mg}$. Doses of pentobarbital in the order of 100 to 200 mg. may produce a definite sedative-soporific or drowsy state in which sleep develops depending upon environmental circumstances. Doses of pentobarbital 5 to 10 times as large may produce a state of semicoma or coma. Thus, the range of dosage between mild sedation, a soporific effect, semicoma, and coma is in the order of twofold to tenfold. What distinguishes reserpine and chlorpromazine from the barbiturates (in addition to certain qualitative differences) is that the range of dosage between mild sedation and coma is extremely large. In man, a dose of chlorpromazine of 50 to $100 \mathrm{mg}$. may produce mild sedation. A tenfold increase in dosage generally produces only a more definite sedative effect. Coma can seldom be produced with the phenothiazines. Patients who ingest several grams of these agents may appear in a semicomatose state with marked hypotension, but even in this state, marked afferent stimuli transiently arouse the patient. This is even more true of reserpine and its derivatives. Compounds such as meprobamate resemble ethyl alcohol and the barbiturates more than the phenothiazines or reserpine. Meprobamate has an intermediate range of doses between those producing mild sedation, a soporific effect, and coma. This wider range of dosage perhaps contributes to its popularity as a tranquilizing drug. Obviously, marked qualita- 
tive differences do exist in the pharmacology of many drugs used clinically to produce tranquilization. Nevertheless, it is suggested that any central nervous system depressant which produces weak sedation in doses smaller than those which produce drowsiness potentially is a clinically useful tranquilizing agent.

There has been a great deal of research on the pharmacology of tranquilizing agents in animals, normal human subjects, and psychiatric patients. In fact, the worldwide literature is so enormous that it is not possible to be even reasonably complete. This review is an attempt to correlate the author's knowledge of animal pharmacology with that in man of a relatively small number of tranquilizing agents, based upon a cursory literature survey. In discussing the pharmacologic actions of certain drugs, it was frequently necessary to refer to data in animals which are not available in man. Whenever appropriate, some studies in which the therapeutic actions of a given compound were compared with one or more standard control agents have also been included in this review.

\section{Substitułed phenothiazines}

Although the phenothiazines have been known for many decades, their use in human medicine was limited until the past 15 years. Historically, phenothiazine itself was employed as a urinary antiseptic and anthelmintic. Toxicity precluded its widespread use. Introduction of promethazine in 1945 as an antihistaminic with sedative properties prompted further synthesis of other derivatives of phenothiazine. This was culminated in 1950 by the successful synthesis of chlorpromazine by Charpentier. Since then, numerous substituted phenothiazines have found a place in clinical medicine.

Structure-activity relationships. Some of the structure-activity relationships of these compounds were reviewed by Friend ${ }^{104}$ in the Current Drug Therapy section of this journal. Several new compounds have since appeared.
The substituted phenothiazines may be classified on the basis of chemical structure."

A. Compounds with a propyl dimethylamino subgroup<smiles>CN(C)C=CN1c2ccccc2Sc2ccccc21</smiles>

Promazine<smiles>CC(C)N1c2ccccc2Sc2ccccc21</smiles>

Trimeprazine<smiles>COc1ccc2c(c1)N(C(C)C(C)C)c1ccccc1S2</smiles>

Methotrimeprazine<smiles>COc1ccc2c(c1)N(CCCN(C)C)c1ccccc1S2</smiles>

Methoxypromazine

*Formulas will usually be drawn as the base in this article. 
<smiles>CNCCCN1c2ccccc2Sc2ccc(Cl)cc21</smiles>

Chlorpromazine<smiles>CCN(C)CCN1c2ccccc2Sc2ccc(C(F)(F)F)cc21</smiles>

Triflupromazine<smiles>CN1CCN(CCCN2c3ccccc3Sc3ccc(S(=O)(=O)N(C)C)cc32)CC1</smiles>

Thioperazine<smiles>CC(=O)c1ccc2c(c1)N(CC=CN1CCN(CCO)CC1)c1ccccc1S2</smiles>

Acetophenazine

B. Compounds with a propyl piperazine subgroup<smiles>CN1CCN(C=CCN2c3ccccc3Sc3ccc(Cl)cc32)CC1</smiles>

Proclorperazine<smiles>CCSc1ccc2c(c1)N(CC=CN1CCN(C)CC1)c1ccccc1S2</smiles>

Thiethylperazine

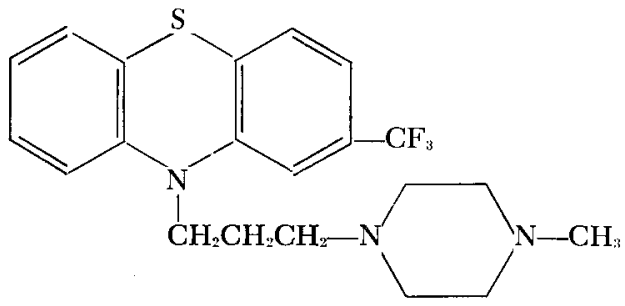

Trifluoperazine<smiles>CCC(=O)c1ccc2c(c1)N(CCCN1CCN(C(CO)CO)CC1)c1ccccc1S2</smiles>

Carphenazine<smiles>OCCN1CCN(CCCN2c3ccccc3Sc3ccc(Cl)cc32)CC1</smiles>

Perphenazine<smiles>CCC(=O)OCCN1CCN(C=CCN2c3ccccc3Sc3ccc(Cl)cc32)CC1</smiles>

Thiopropazate 


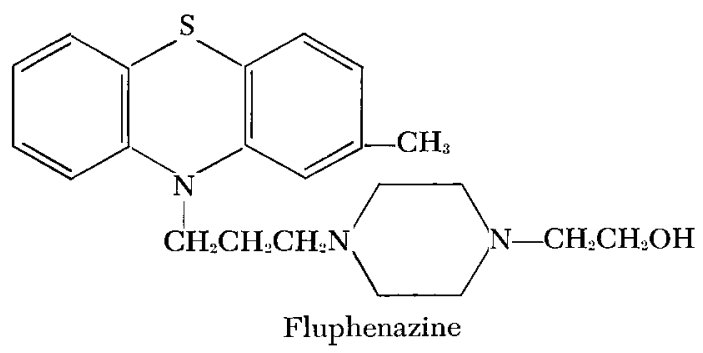

C. Compound with a methyl piperidyl subgroup<smiles>CN1CCCC(CN2c3ccccc3Sc3ccccc32)C1</smiles>

Mepazine

D. Compound with an ethyl piperidyl subgroup

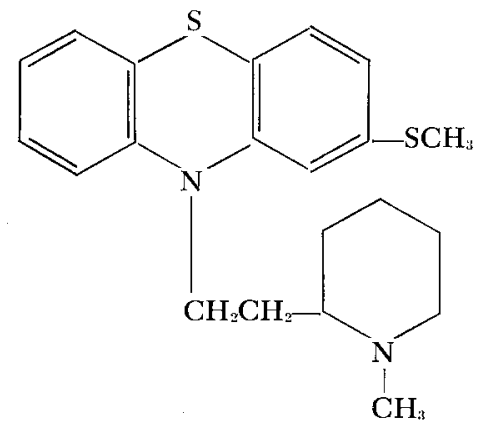

Thioridazine

E. Compound with a propyl piperidyl subgroup

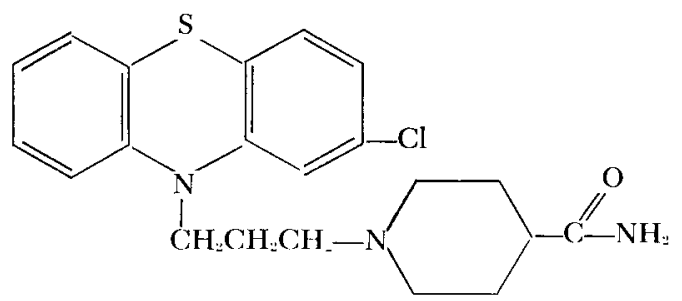

Pipamazine
F. Compound with a methyl pyrrolidyl subgroup<smiles>CCCCC1C2CCCCC2CN1C</smiles>

G. Compounds with an ethyl dimethylamino subgroup<smiles>CC(C)N1c2ccccc2Sc2ccc(Cl)cc21</smiles>

Chlorpromethazine<smiles>CCC(=O)c1ccc2c(c1)N(C(C)C(C)C)c1ccccc1S2</smiles>

Propiomazine<smiles>CNC(C)CN1c2ccccc2Sc2ccccc21</smiles>

Promethazine 
H. Compounds with an ethyl diethylamino subgroup<smiles>CCN(CC)CCN1c2ccccc2Sc2ccccc21</smiles>

Diethazine<smiles>CCN(CC)C(C)CN1c2ccccc2Sc2ccccc21</smiles>

Ethopropazine

I. Compound with an ethyl pyrrolidyl subgroup

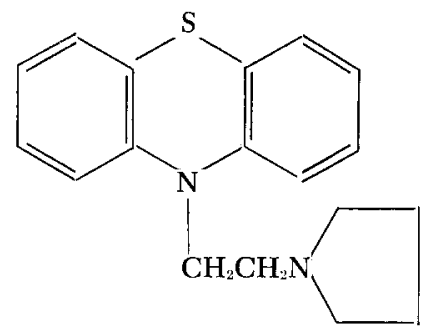

Pyrathiazine

The substituted phenothiazines have a very complex pharmacology and have effects at many different sites in the body.

They act in the central nervous system, producing (1) sedation, (2) block of conditioned avoidance behavior, (3) antiemetic effects, (4) alteration of temperature regulation, (5) alteration of skeletal muscle tone, (6) antipruritic effects, (7) analgesic effects, (8) facilitation of seizure discharge, and (9) endocrine changes.

They act in the autonomic nervous sys- tem, producing (1) cholinergic blocking effects at nicotinic and muscarinic receptor sites, (2) adrenergic blocking effects (alpha type), (3) adrenergic potentiating effects (especially norepinephrine), (4) antihistaminic effects, and (5) antiserotonin effects.

They potentiate the action of various drugs.

Finally, they induce local anesthesia.

The substituted phenothiazines differ quantitatively and qualitatively in the extent of each of these pharmacologic effects. They also vary in the degree of untoward effects, including agranulocytosis, photosensitivity, extrapyramidal symptoms, jaundice, and pigmentary retinopathy. It is not easy to make valid generalizations with regard to their structure-activity relationships. ${ }^{117}$ A few principles do emerge which may facilitate retention of some of the known facts about the phenothiazines.

Generally, compounds which contain the $-\mathrm{C}-\mathrm{C}-\mathrm{N}$ side chain in position 10 with two carbons between the nitrogens have increased antihistaminic properties (promethazine and pyrathiazine). Compounds with a $-\mathrm{C}-\mathrm{C}-\mathrm{C}-\mathrm{N}$ side chain in position 10 with three carbons between the two nitrogens have increased effects in blocking conditioned avoidance behavior as well as increased adrenergic blocking properties. Substitution of Cl for a hydrogen in position 2 of the phenothiazine ring for compounds with three carbons between the two nitrogens usually increases potency for depressing conditioned avoidance behavior (chlorpromazine versus promazine). On the other hand, a 2-Cl substitution in compounds with two carbons between the two nitrogens reduces potency for depressing conditioned avoidance behavior (2-chlorpromethazine versus promethazine). An $\mathrm{OCH}_{3}$ group in position 2 is almost as effective as a $\mathrm{Cl}$ substitution (methoxypromazine versus chlorpromazine). The $\mathrm{CF}_{3}$ substitution in position 2 
produces a marked increase in potency (triflupromazine, trifluorperazine, and fluphenazine). Other position 2 substitutions which also increase potency include<smiles></smiles>

(acetophenazine). The 2-thio-substituted compounds appear to produce fewer untoward extrapyramidal effects (thioridazine and thiethylperazine versus proclorperazine).

Substitution of a piperazine group in place of the terminal dimethylamino moiety increases potency for central effects, including extrapyramidal symptoms, for example, proclorperazine versus chlorpromazine. A further increase in potency is obtained by replacing a $\mathrm{Cl}$ with a $\mathrm{CF}_{3}$ group (proclorperazine versus trifluoperazine). Substitution of $-\mathrm{CH}_{2} \mathrm{CH}_{2} \mathrm{OH}$ for the terminal $\mathrm{CH}_{3}$ group on piperazine also results in a slight increase in potency (perphenazine versus proclorperazine). Even greater central potency, including extrapyramidal symptoms, is obtained by substituing $\mathrm{CF}_{3}$ for $\mathrm{Cl}$ (fluphenazine versus perphenazine). Quaternization of the terminal nitrogen of chlorpromazine decreases lipid solubility, reducing penetration through the blood-brain barrier. This results in a compound with markedly diminished central nervous system effects. The tissue distribution of certain quaternized phenothiazines is markedly altered, with much less appearing in brain than in the case of their tertiary analogues. ${ }^{7}, 32,125$

Some agents with a terminal $\mathrm{N}$-diethyl group possess interesting antiparkinsonian effects and increased nicotinic cholinergic blocking properties (diethazine and ethopropazine). Both drugs also possess antihistaminic properties. Phenothiazines used especially for their antihistaminic properties include promethazine and pyrathiazine. Clinically useful antipruritic effects have been ascribed especially to trimeprazine and methdilazine. These latter agents ap- pear to be less effective tranquilizers. Most of the substituted phenothiazines with three carbons between the terminal nitrogens also possess marked antiemetic effects. In small doses, they depress the chemoreceptor trigger zone, but in larger doses, they have a direct depressant effect on the vomiting center. ${ }^{111}$ Thiethylperazine has been claimed to be effective in depressing both of these medullary vomiting areas, but further data are necessary to evaluate its usefulness and specificity. Although chlorpromazine and promethazine are not clinically effective analgesic agents, Lasagna and De Kornfeld ${ }^{194}$ have data on a new phenothiazine, methotrimeprazine, indicating that milligram for milligram in doses of 10 to $15 \mathrm{mg}$. subcutaneously given to bedridden postoperative and postpartum patients, it was as effective as morphine. However, doses of $25 \mathrm{mg}$. of methotrimeprazine given orally to ambulatory patients produced untoward effects such as dizziness, fainting, drowsiness, and vomiting (also seen in 1 patient with a placebo) but no analgesia superior to a placebo. Sedation and postural hypotension occur with this phenothiazine. They suggest that this and other phenothiazines be studied further for analgesic properties.

The ramifications of the structure-activity relationship of the phenothiazines appear to be limited only by available discrete screening tests and chemical imagination. The rather stereotyped structure-activity relationship of compounds clinically available attests probably to the lack of suitable animal screening tests for unique therapeutically desirable properties in man. As such screening procedures are developed, one can probably anticipate many more interesting phenothiazine analogues. It would appear that the numerous pharmacologic effects of the substituted phenothiazines can be partially separated by appropriate "molecule manipulation." This is in sharp contrast to the multiplicity of other drugs with which milligram potency and duration of action are the primary pharmacologic variables altered by minor 
<smiles>CN(C)CCN1c2ccccc2CCc2ccccc21</smiles>

Imipramine<smiles>CNCCCN1c2ccccc2Sc2cccnc21</smiles>

Prothipendyl

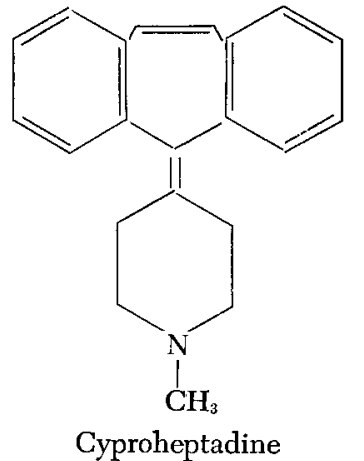

Cyproheptadine<smiles>CNCC=CC1c2ccccc2CCc2ccccc21</smiles>

Amitriptyline<smiles>CN(C)CC=C1c2ccccc2Sc2ccc(Cl)cc21</smiles>

Chlorprothixene chemical substitutions, for example, the numerous thiazide diuretics. The appearance of newer compounds related to the phenothiazines is ample evidence of the fruitfulness of the grab bag attempts in molecular manipulation. The appearance of imipramine and amitriptyline as psychic energizers is the direct result of minor alterations in the structure of promazine. These agents (especially imipramine) appear to have a more marked norepinephrine-potentiating action than the parent phenothiazine. Other alterations of the phenothiazine ring may result in compounds with primarily sedative effects, as in the case of prothipendyl, which is also modeled after promazine, and chlorprothixene, which is modeled after chlorpromazine. Cyproheptadine is another analogue with serotonin-blocking and histamine-blocking properties of value as an antipruritic.

The choice of a phenothiazine to use as a tranquilizing agent depends upon the type of patient, the potency, and possible untoward effects of the drug. In usual therapeutic doses, frequently, it matters little which of the many phenothiazines with a $-\mathrm{CH}_{2} \mathrm{CH}_{2} \mathrm{CH}_{2} \mathrm{~N}$ substitution are used. For example, Hollister, Caffey, and Klett $^{143}$ compared the complications in schizophrenic patients after 12 weeks of treatment with chlorpromazine, triflupromazine, mepazine, proclorperazine, perphenazine, and phenobarbital. Many abnormal symptoms and signs which were thought to be associated with phenothiazine therapy also occurred with phenobarbital. For example, leukopenia was not significantly more frequent from the phenothiazines than from phenobarbital! Changes in body temperature, pulse rate, and blood pressure were uncommon, with no significant differences in frequency of occurrence among various drugs used. Obviously, this lack of undesirable effects is in part related to the dosage used.

Behavioral effects. The administration of chlorpromazine and other phenothiazines to man produces depression of the central nervous system. The subject may become sleepy, but arousal to various afferent stimuli usually occurs easily. After chlorpromazine, psychotic patients appear less severely disturbed. Generally, this antipsychotic ef- 
fect is more likely to be seen on chronic administration than acutely. Acute drug administration does, however, produce a quieting effect in grossly agitated and disturbed patients. Recently, Tuteur, Stiller, and Glotzer ${ }^{311}$ reviewed the results of chlorpromazine administration over a 5 year period to once denudative, incontinent, combative, destructive psychotic female patients with lengthy hospitalizations. At the end of the 5 year period in which 913 patients were treated, 273 were discharged. Of the latter, 75 returned to the hospital; 28 relapsed while taking chlorpromazine diligently. Extensive controls were not available. Nevertheless, it would appear that chlorpromazine has antipsychotic effects in some patients. Because of hospital discharges, the original treatment area of 814 beds could be decreased by removing 154 beds permanently. Somewhat similar results were described by $\mathrm{Kris}^{189}$ in a 5 year follow-up study involving patients given a variety of pharmacologic agents, predominantly phenothiazines.

It is a common clinical impression that the sedative or antipsychotic effects of the substituted phenothiazines are qualitatively different from those of other central nervous system depressants such as the barbiturates. There is good evidence to support this widespread impression. The sites and mechanisms of action of chlorpromazine are certainly uniquely different from those of the barbiturates. ${ }^{83}$ An interesting experiment which illustrates the differences in pharmacology of chlorpromazine and phenobarbital was reported by Lasagna and $\mathrm{McCann}^{195}$ with mice. The toxicity of amphetamine is much less for isolated than for aggregated mice. In single isolated mice, neither phenobarbital nor chlorpromazine in the doses used had an appreciable effect on the $\mathrm{LD}_{50}$ of amphetamine. In grouped animals, however, chlorpromazine gave significant protection against amphetamine, while phenobarbital did not unless anesthetic doses were used. Further evidence of the differences between chlorpromazine and a barbiturate on objective behavioral tests has been provided by Brady, Nurnberger, and Tausig. ${ }^{39}$ These investigators attempted an experimental analysis of some young adult psychotic patients using operant conditioning techniques now widely used to analyze animal behavior. In a preliminary study the details of which unfortunately were not presented, they compared the effects of single oral doses of chlorpromazine and secobarbital given to psychotic subjects who developed a stable operant behavioral pattern. Chlorpromazine affected the fixed interval mean delays and the tendency for delays in most subjects in the same direction as clinical improvement had occurred over a long period of treatment. Secobarbital had no such effect.

Clinical studies on the comparative efficacy of the substituted phenothiazines and barbiturates also support the notion of a unique antipsychotic effect of the phenothiazines. For example, Casey and coworkers, ${ }^{57}$ in an extensive double blind study involving various cooperating veterans administration hospitals, reported on the therapeutic effectiveness of phenothiazines in contrast to phenobarbital and a placebo in 692 schizophrenic men. Twelve week periods were used for the drug treatments. Chlorpromazine (400 mg. daily) was more effective than promazine ( 400 mg. daily), while phenobarbital (200 mg. daily) and placebo (lactose) were relatively ineffective. When phenothiazine medication followed the control medications (phenobarbital or lactose), clinical improvement increased. When the control medication was given after the phenothiazines, however, the gains obtained after the phenothiazines persisted for the additional 12 week period of the study. This may be related to the fact that phenothiazine metabolites may still be present for this length of time after stopping medication (see section on metabolic fate). The results of this study, furthermore, support the clinical impression that antipsychotic effects of phenothiazines are unrelated to drowsiness to which tolerance develops. 
Generally, chlorpromazine is not considered a soporific agent, although Cutler, Monroe, and Anderson, ${ }^{67}$ reported that 150 mg. of chlorpromazine administered three times a day to schizophrenics produces somnolence. Kornetsky, Vates, and Kessler ${ }^{186}$ compared the effects of 100 and 200 mg. of chlorpromazine, $200 \mathrm{mg}$. of chlorpromazine and $200 \mathrm{mg}$. of secobarbital, and a placebo given orally to normal subjects before bedtime. Both drugs produced a significant increase in sleeping time. Of considerable interest is that both drugs also significantly impaired the subjects' performance on various psychologic tests the following morning, 14 to 15 hours after medication. This would indicate a possible hangover effect to both agents. Kornetsky and co-workers ${ }^{185}$ have noted that there is tolerance development to the effects of chlorpromazine (100 and $200 \mathrm{mg}$. twice a day for 11 days) on some behavioral tests after chronic administration to schizophrenic patients. On the other hand, chronic administration of secobarbital ( 200 mg.) still significantly reduced the level of performance. Single doses of 100 and 200 mg. of chlorpromazine caused less impairment in schizophrenics than in normal subjects. In contrast, single doses of secobarbital caused a similar degree of impairment of performance in schizophrenics and normal subjects. Kornetsky ${ }^{184}$ has summarized many of his previously reported studies, pointing out in particular the differences in the effects of chlorpromazine compared to other psychoactive drugs depending upon whether the drugs are given chronically or acutely. Furthermore, individual differences may affect the behavioral actions of the drugs. He has proposed the hypothesis (and offered some data in support) that the responder to one drug is also a responder to other drugs. This also applies to drugs producing opposite effects. Kornetsky showed that human subjects impaired the most by chlorpromazine tended to be stimulated the most by D-amphetamine. Although not great $(-0.48)$, the correlation coefficient was statistically significant.
Further evidence of tolerance development to the sedative effects of chlorpromazine has been provided by Rosner, Ameen, and Kolers. ${ }^{267}$ These investigators compared the effects of chronic administration of chlorpromazine, mephobarbital, and a placebo on tests of alertness in 11 acutely schizophrenic patients. A crossover design was used with drugs alternating after 6 weeks. The subjects received $100 \mathrm{mg}$. of chlorpromazine four times a day and 200 $\mathrm{mg}$. of mephobarbital four times a day. After chronic administration of both drugs, there was no deficit of performance on various tests of alertness. This is to be contrasted with the results reported by Primac, Mirsky, and Rosvold, ${ }^{258}$ that single dose administration of chlorpromazine does adversely affect the performance of normal subjects on a "continuous performance test" of alertness. Interestingly, secobarbital did not have a deleterious effect. Similar effects were reported subsequently by Mirsky, Primac, and Bates. ${ }^{227}$

Chlorpromazine usually markedly reduces motor activity in man and animals. Pathologic motor activity in psychotic patients is significantly reduced by $600 \mathrm{mg}$. of chlorpromazine daily in contrast to a placebo in severely disturbed patients. ${ }^{67}$ It is of interest that the drug also significantly increased somnolence although this did not appear to be great enough to account for the decrease in pathologic activity. In view of the fact that there is evidence for tolerance development to the sedative effects of chlorpromazine but not to the antipsychotic effects, it is of interest to note that in rats, Irwin ${ }^{154}$ has observed tolerance to develop to the depression of conditioned avoidance by chlorpromazine or perphenazine within 2 weeks after chronic daily administration. No tolerance developed to the depression of locomotor activity. Irwin suggested on this basis that locomotor depressant effects in rats correlated better with antipsychotic activity than depression of conditioned avoidance. On acute administration, the two phenomena were highly correlated in a series of 
eight substituted phenothiazines, with the exception of promazine. ${ }^{155}$ The latter had a greater depressant effect on locomotor activity than on conditioned avoidance.

Cook and Weidley determined the specificity of chlorpromazine in blocking instrumental conditioned avoidance in rats. The conditioned avoidance response was selectively blocked by chlorpromazine, reserpine, and morphine, in contrast to the effects of barbital and methylparafynol. Pentobarbital and meprobamate affected the conditioned response in doses producing neurologic symptoms. Hertz ${ }^{135}$ reviewed much of the literature on the effects of a wide variety of psychopharmacologic agents on conditioned avoidance in animals. The available literature further supports the notion that chlorpromazine, reserpine, and morphine produce more selective depressant effects on avoidance than on escape behavior. Winsor ${ }^{333}$ reported that in man, various phenothiazines, including chlorpromazine, proclorperazine, methoxyphenothiazine, and mepazine, prevented the development of/or blocked conditioned vasoconstriction in the fingers but not the unconditioned response to an electric shock. Pentobarbital and a placebo had no effect on the development or inhibition of conditioned vascular responses. No doses or descriptions of subjects, however, were included in this report.

Ader and Clink ${ }^{2}$ determined the effects of chlorpromazine on the acquisition and extinction of a conditioned-avoidance shuttle box type of response in rats. Chlorpromazine treatment increased the number of trials required to attain a given criterion of learning and decreased the resistance to extinction. The role of motor retardation rather than a specific effect of chlorpromazine on acquisition or extinction was not determined. Schneider and Costiloe $^{275}$ studied in man the effects of chlorpromazine, amobarbital, and phenidylate on the spontaneous recovery and extinction of the galvanic skin response to a previously conditioned stimulus using electric shock as the unconditioned stim- ulus. Chlorpromazine inhibited reconditioning, while phenidylate facilitated it. Amobarbital inhibited spontaneous recovery and also tended to inhibit reconditioning. Truax ${ }^{308}$ was able to show that in contrast to placebo, $40 \mathrm{mg}$. of chlorpromazine facilitated the rate of eyelid conditioning in human volunteers. During extinction, neither the drug nor placebo caused decrement per se, but the change from either produced block of the emotionally conditioned response. A related study by the same investigator and associates $^{285}$ compared the effects of chlorpromazine (30 mg.) and a placebo given orally on motion studies of gait, manual performance in handwriting, object manipulation, and panel control operations. Each of 40 human subjects acted as their own controls. Chlorpromazine was shown to significantly increase the duration of fine manipulative movements after learning progressed, without affecting travel movements or manipulative movements early in learning. The drug appeared to interfere or reduce the efficiency of fine motor control. Traugott and Balnov ${ }^{307}$ determined the effects of chlorpromazine on conditioned and unconditioned reflexes as well as reaction and association responses in various patients. Chlorpromazine impaired conditioned activity and lessened simple, unconditioned reflexes. They also claimed their patients appeared less tense, more accessible, and better oriented after the drug. Alexander ${ }^{5,6}$ also determined the effects on various drugs in man using the classic Pavlovian method of pairing a tone with an electric shock. He recorded the galvanic skin response. Chlorpromazine and related phenothiazines depressed the conditioned as well as the unconditioned responses.

A few studies have been made on the objective behavioral effects in man of various other phenothiazines. Benjamin, Ikai, and Clare $^{25}$ determined the effects of 10 $\mathrm{mg}$. of proclorperazine and $10 \mathrm{mg}$. of phenobarbital given orally to human volunteers on various psychologic, psychomotor, 
and muscular performance tests. The results obtained were compared to those with a control group receiving no drug. Tests of hearing, pain perception, and mental performance showed no specific drug-induced changes. Tests involving muscular coordination, however, showed a decrease in performance as did tests of muscular exercise. The amplitude of the patellar reflex was decreased by proclorperazine, whereas phenobarbital had no effect. Proclorperazine significantly prolonged the combined simple and choice reaction times and depressed pursuit rotor performance more than phenobarbital. Wilson and Huby ${ }^{331}$ compared the effects of thiopropazate ( 20 mg.), secobarbital (120 mg.), caffeine ( 300 mg.), and a lactose placebo in normal students using subjective and objective questionnaires. The subjects were acquainted with the pharmacologic actions of the drugs and were asked to guess which agent they received. When the subjects guessed correctly, the drug effects were more marked than when they guessed incorrectly. It was felt that this was evidence that the pharmacologic actions of the drugs could be modified by anticipation of their effects. Thiopropazate could not be distinguished from secobarbital. Caffeine was preferred; it tended to cause wakefulness but was preceded by a period of drowsiness.

Klerman and DiMascio ${ }^{172}$ compared the effects of single doses of chlorpromazine, promethazine, perphenazine, and trifluoperazine in 36 normal human subjects using a double blind design. Various psychologic tests were used: alertness, psychomotor speed, steadiness, intellectual tasks, and mood. These were administered before and 2,4 , and 7 hours after drug ingestion. Of considerable importance was the observation that the ethyl and propyl dimethylamine derivatives (promethazine and chlorpromazine) produced a marked decrease in psychomotor function, impairment of intellectual tasks, and drowsiness. On the other hand, the propyl piperazine derivatives (perphenazine and trifluoperazine) had the opposite effects and elevated mood. A more detailed report of essentially the same study was given by DiMascio, Havens, and Snell. ${ }^{78}$ Chlorpromazine and promethazine $(25,50,100$ or $200 \mathrm{mg}$. per day) and perphenazine and tripfluoperazine $(2,4,8$, or $16 \mathrm{mg}$. per day) were all given orally. Chlorpromazine and promethazine produced psychomotor inhibition, mental confusion, alterations in autonomic function, and sedation. In contrast, perphenazine and trifluoperazine resulted in minimal changes in all of these measures. At the highest dose levels of these two drugs, the subjects actively tried to go to sleep in order to eliminate some of the inner stimulation they noted. In contrast, after chlorpromazine and promethazine, the subjects reported that sleep seemed to be forced on them. Chlorpromazine and promethazine produced pupillary constriction, while perphenazine and trifluoperazine caused dilatation. When impairment of performance was noted with any of the drugs, there tended to be associated pupillary constriction. Chlorpromazine was the only drug which tended to alter blood pressure. The authors suggested that many of the effects observed were perhaps more related to the untoward effects of the phenothiazines than to their therapeutic or antipsychotic actions.

Effects on the human electroencephalogram. Numerous electroencephalographic studies have been published on the substituted phenothiazines. For purposes of brevity, only a few will be referred to in this review. Dobkin, Gilbert, and Lamoureux $^{81}$ showed that after intravenous administration of 0.3 to $2 \mathrm{mg}$. per kilogram of chlorpromazine to unanesthetized patients and volunteers, the electroencephalogram resembled that of light drowsiness. The subject could be easily aroused. The electroencephalographic picture of drowsiness was represented by irregular, 5 to 6 cycles per second, low voltage theta waves in the central midline or midvertex area. The $\mathrm{K}$ complex which occurs as part of the electroencephalographic arousal response in 
drowsiness or sleep was also found. Of pharmacologic interest, neostigmine, caffeine, amphetamine, and edrophonium did not antagonize these electroencephalographic changes. Szatmari ${ }^{303}$ observed that $50 \mathrm{mg}$. of chlorpromazine given intravenously produced the following changes in the human electroencephalogram: marked muscle relaxation and disappearance of muscle artifact, (2) a decrease of $1 \frac{1}{2}$ cycles per second in the alpha frequency and a 30 per cent increase in voltage, (3) an increase in temporal and parietal 5 to 6 cycles per second theta activity, (4) drowsiness or light sleep patterns, and (5) in epileptics, increased sensitivity to hyperventilation. The tendency for increased synchronization and normalization of the electroencephalogram was taken as evidence that chlorpromazine had a very mild depressant action on the reticular activating system. Some of the animal evidence would indicate that chlorpromazine does indeed have a mild depressant effect on this system. Glotfelty and Wilson $^{112}$ compared the effects of chronic administration (greater than 4 days) of chlorpromazine, promazine, and meprobamate on the electroencephalographic arousal response in various psychiatric patients to several afferent stimuli including visual, auditory, tactile, and nociceptive stimuli. Doses of 200 to $400 \mathrm{mg}$. of chlorpromazine or promazine and $1,600 \mathrm{mg}$. of meprobamate given orally daily for at least 4 days had no effect on electroencephalographic arousal responses in man. When promazine was given intravenously in doses of 75 to $200 \mathrm{mg}$., however, electroencephalographic arousal responses were reduced markedly in duration. These data might be interpreted to mean that chronic oral administration leads to tolerance so that depressant effects on electroencephalographic arousal were not observed. On the other hand, an acute intravenous dose of promazine (and probably chlorpromazine) does depress electroencephalographic arousal in man. Interestingly, Himwich, Rinaldi, and Willis ${ }^{138}$ proposed from an electroencepha- lographic study of a limited series of phenothiazines in rabbits that those phenothiazines which failed to block electroencephalographic arousal to painful stimuli on acute administration were inferior to chlorpromazine in managing psychotic patients. Paul-David and Unna ${ }^{243}$ compared a series of substituted phenothiazines in a quantitative manner on electroencephalographic arousal of the cat elicited by stimulation of the brain stem reticular formation. All drugs were given intravenously. The minimal effective doses to depress the electroencephalographic arousal response for the drugs were: chlorpromazine, 0.5 mg. per kilogram; chlorpromazine sulfoxide, $5 \mathrm{mg}$. per kilogram; trifluoperazine, $0.02 \mathrm{mg}$. per kilogram; perphenazine, 0.1 mg. per kilogram; and triflupromazine, 0.05 mg. per kilogram. Promazine and thioridazine were less effective, and prochlorperazine was as effective as chlorpromazine in depressing the electroencephalographic arousal response.

Swain and Litteral ${ }^{299}$ obtained electroencephalographic recordings during a double blind study of the effects of chlorpromazine given chronically before, during, and various periods after cessation of medication to senile, brain-damaged patients. Electroencephalographic abnormalities consisting of slowing persisted in some patients for 3 months or longer after the medication was discontinued. In contrast, Jörgensen and Wulff ${ }^{159}$ studied the electroencephalographic effects of chlorpromazine and its withdrawal in a younger group of nonorganic psychiatric patients. Electroencephalographic changes disappeared 10 days after chlorpromazine withdrawal. It would appear that senile, brain-damaged patients show residual chlorpromazine effects much longer than younger patients without organic brain disease.

Fink has proposed that increased electroencephalographic synchronization and a shift of frequencies to the delta range commonly are associated with tranquilization induced in psychiatric patients by the phenothiazines (particularly chlorproma- 
zine and promazine). He distinguishes these electroencephalographic effects with those produced by the barbiturates, which regularly induce an increase in fast activity and synchrony, and sympathomimetics such as amphetamine, which increase fast activity without increased synchrony. In contrast, desynchronization is prominent after diethazine, lysergic acid diethylamide, and benactyzine.

Effects on skeletal motor systems. On acute administration, chlorpromazine depresses skeletal muscle tone. For example, Sheatz ${ }^{282}$ was able to show that chlorpromazine abolished extensor hypertonicity and hyperreflexia in four of five dogs with midcollicular brain stem transections. In 3 human patients with irreversible brain damage showing decorticate hypertonicity, $100 \mathrm{mg}$. of chlorpromazine intramuscularly caused marked skeletal muscle relaxation.

Basmajian and Szatmari ${ }^{18,} 19$ were able to confirm the fact that chlorpromazine can reduce skeletal muscle spasticity in some patients with neurologic lesions. Patients showing spasticity caused by lesions of upper motor neurons were dramatically improved after chlorpromazine. Of considerable theoretic interest was the fact that the drug reduced the tremor and rigidity of parkinsonism for about 2 hours. These investigators postulated that the agent depressed bulbar facilitatory areas in the brain stem. Henatsch and Ingvar ${ }^{134}$ showed in cats that chlorpromazine has a marked depressant effect, especially on central gamma motor systems. They suggested that forms of spasticity and rigidity which are reduced by chlorpromazine are probably mediated over the gamma motor system. The depressant effect of chlorpromazine on skeletal muscle tone is apparently due to an action on the brain stem. ${ }^{151}$

Chlorpromazine has been reported to be effective in controlling the skeletal muscle spasms of tetanus in man. ${ }^{15,198}$

Laurence and Webster ${ }^{199}$ have observed, however, that after repeated doses of chlorpromazine over an 8 hour period to rabbits with local tetanus, tolerance developed rapidly. Subsequently, larger doses had no suppressant effect and usually facilitated the tetanus spasms. After the depressant effect of a large dose of chlorpromazine wore off, it was usually followed by enhanced activity. If a second dose was given during the next 24 hours, there was an immediate activation of the response which could be abolished by a barbiturate or mephenesin. Levomepromazine was a more effective depressant than chlorpromazine. In a human case of tetanus, levomepromazine gave symptomatic relief in a dose of $25 \mathrm{mg}$. intravenously and $400 \mathrm{mg}$. daily on succeeding days.

The effects of the phenothiazines on the extrapyramidal system are well known. Generally, these effects are considered to be incidental to the antipsychotic actions of the phenothiazines, although some have regarded the two to be related. This impression is strengthened by the fact that the more potent antipsychotic agents show a greater tendency to produce extrapyramidal symptoms. Goldman ${ }^{115}$ studied in 5,000 state hospital patients the extrapyramidal syndromes induced by various drugs including the phenothiazines, reserpine, and related compounds. There appeared to be no relationship between dosage, antipsychotic effect, and induced parkinsonism. Phenothiazines with a position 10 propyl dimethylamino substitution and a position 2 chlorine or trifluoromethyl group induced parkinsonian phenomena. Among the position 10 propyl piperazine derivatives, extrapyramidal symptoms were induced by the sulfondimethylamide, trifluoromethyl, and chlorine substitutions in position 2 . The neurologic effects of tetrabenazine and haloperidol outweighed their therapeutic effectiveness. Goldman felt that the apparent correlation between the ability to induce parkinsonism and to suppress psychotic symptoms was highly misleading.

Actions on cardiovascular system. The descriptions of Dobkin, Gilbert and Lamoureux $^{81}$ on the pharmacologic effects of intravenous chlorpromazine ( 0.3 to $2 \mathrm{mg}$. per kilogram) to healthy human subjects 
are classic. After chlorpromazine, the subjects became drowsy, listless, pale, calm, and apathetic. Their skin was dry and warm. Horner's syndrome was present bilaterally. The subjects had a feeling of motor weakness and were chilly and thirsty. The axillary, oral, and rectal temperatures decreased 2 to $4^{\circ} \mathrm{F}$. At the same time, the temperatures in the extremities rose. Arterial blood $\mathrm{pH}$ and sodium, potassium, chloride, and phosphate levels did not change, but the bicarbonate content fell. Arterial oxygen, carbon dioxide content, hematocrit, and hemoglobin determinations showed no change. Arterial blood sugar rose, while blood urea nitrogen fell. The subjects showed orthostatic hypotension, and the heart rate rose slightly. The tidal volume was depressed, but the rate of respiration increased and was irregular. Doses of 1 to $2 \mathrm{mg}$. per kilogram of chlorpromazine produced definite respiratory depression and in 1 subject dyspnea. The electroencephalogram showed a normal sleep pattern. The subjects were easily aroused by afferent stimuli and often showed a hyperactive response. After chlorpromazine, there was a marked decrease in gastric secretions, but insulin caused a marked response, indicating that the vagus was functional. In a small number of cases, the movements of athetosis and chorea were suppressed, nausea and vomiting were controlled, and chronic prolonged pain was controlled with chlorpromazine and smaller doses of meperidine. Foster and colleagues ${ }^{100}$ studied the effects of chlorpromazine given intravenously in doses of 5 to $25 \mathrm{mg}$. in conscious as well as anesthetized subjects. These doses caused varying degrees of tachycardia and postural hypotension. By means of plethysmography, they were able to show that chlorpromazine produced vasodilation in the hand, calf, and forearm on intravenous and intra-arterial injection. Blood flow was further increased in the hand after block of the brachial plexus by local anesthesia. Chlorpromazine reduced the hypertension induced by intravenous norepinephrine and blocked the reflex bradycardia. Vasoconstriction following intra-arterial infusion of norepinephrine, however, was not reduced, but that to epinephrine was. Epinephrine reversal was not observed. It would appear that chlorpromazine in the doses used had a weak alpha adrenergic blocking action and a possible ganglionic blocking effect. Using larger doses in animals, numerous investigators have shown that chlorpromazine produces alpha adrenergic block. There are marked differences among the various phenothiazines in this regard. ${ }^{89}$ The metabolic effects of epinephrine, however, are not blocked. Gupta, Patel, and Joseph ${ }^{122}$ have shown that chlorpromazine alone produces significant hyperglycemia in the rabbit. A similar effect in man was observed by Dobkin, Gilbert, and Lamoureux. ${ }^{81} \mathrm{Al}$ though chlorpromazine blocked the pressor response, it did not block the hyperglycemic response to epinephrine.

Moyer $^{230}$ compared the animal and human pharmacology of chlorpromazine. In general, the pharmacologic effects are similar. In man, chlorpromazine reduced the blood pressure of normotensive as well as hypertensive patients. Intravenous administration produced more marked hypotension than via the intramuscular or oral route. The hypotension was much less marked in recumbent patients than when they were erect. Associated with the hypotension was a decrease in cerebral blood flow. Cerebral oxygen uptake is not affected by chlorpromazine inasmuch as the arteriovenous oxygen difference increased during these induced circulatory changes. With doses of 50 to $100 \mathrm{mg}$. intravenously, adrenergic block was incomplete. Norepinephrine pressor effects were reduced to about onehalf. On chronic administration, tolerance developed to the hypotensive effect of chlorpromazine so that even doses as large as $2 \mathrm{Gm}$. given orally did not lower blood pressure. In man, chlorpromazine, either acutely or chronically, does not appear to cause any consistent alteration of glomerular filtration rate, renal plasma flow, or electrolyte excretion patterns. This is in 
contrast to some data in anesthetized dogs in which in large doses ( 3 to $5 \mathrm{mg}$.) of chlorpromazine may elicit a moderate diuretic response with an increase in sodium excretion. Ginsburg and Duff ${ }^{110}$ compared the effects of intra-arterial and intravenous epinephrine before and after intra-arterial and intravenous chlorpromazine on the hand blood flow of human volunteers. Before chlorpromazine, epinephrine caused a decrease of hand blood flow; after chlorpromazine, the basal flow increased markedly. Subsequently, epinephrine caused a much smaller decrease in hand blood flow. No reversal of epinephrine vasoconstriction of the hand was found, no matter whether intra-arterial (1.2 mg.) or intravenous (50 mg.) chlorpromazine was used. Chlorpromazine via both routes was more effective in antagonizing intra-arterial than intravenous epinephrine. The data on lack of epinephrine reversal are in agreement with the studies of Foster and colleagues, ${ }^{100}$ who also showed a reduction of peripheral epinephrine vasconstriction but inconclusive evidence of epinephrine reversal. Duff and Ginsburg $^{87}$ subsequently did a similar study using intra-arterial and intravenous injections of norepinephrine and chlorpromazine on the hand blood flow of human volunteers. The infusion of chlorpromazine into the brachial artery caused a marked reduction of the vasoconstrictor response to norepinephrine. The effect was attributed primarily to a direct vasodilator action but also to block of norepinephrine. In comparing these results with those previously obtained with epinephrine, these investigators pointed out that chlorpromazine appears to be more effective in reducing the vasoconstriction of the blood vessels of the human hand by norepinephrine than epinephrine. In fact, the reduction in the constrictor response to epinephrine could be wholly accounted for by the vasodilatation produced by chlorpromazine. In various laboratory animals, chlorpromazine may reduce or reverse the pressor effects of epinephrine and reduce those of norepinephrine. ${ }^{68,180}$ Rarely, an enhancement of the norepinephrine blood pressure response has been observed, especially with small doses of chlorpromazine.

The discrepancies between the animal and available human data probably can be accounted for more on the basis of differences in the vascular beds studied and anesthesia than of species differences. Lanzoni, Li, and Etsten ${ }^{192}$ determined the circulatory effects of $0.15,0.25$, and $0.35 \mathrm{mg}$. per kilogram of proclorperazine given intravenously to various patients. Within 2 minutes after administration of proclorperazine, there was a 6 to 10 per cent fall in diastolic and systolic blood pressure and a 5 to 10 per cent increase in heart rate. These effects lasted only 10 to 15 minutes. A slight decrease ( 10 per cent) in the blood pressure rise to the Valsalva maneuver was noted after proclorperazine.

Endocrine effects. Numerous investigators have described the endocrine changes induced by the phenothiazines. The effects obtained depend upon the dose, duration of treatment, and species. In large doses, chlorpromazine and reserpine ${ }^{17}$ and perphenazine $^{316}$ induce a pseudopregnancy response in animals. In therapeutic doses in female patients, Whitelaw ${ }^{327,328}$ reported that $25 \mathrm{mg}$. twice daily of chlorpromazine 1 to 3 days prior to expected ovulation produced a delay in ovulation and menstruation as judged by suction biopsy findings and basal body temperatures. When the medication was started just after menstruation, prolongation of the cycle was not noted. He postulated that chlorpromazine depresses the hypothalamus, interfering with pituitary gonadotropin output, when it is given just prior to ovulation. The fact that daily use of the drug from the time of menses to the next expected ovulation did not interfere with the latter suggests that tolerance may develop to this phenomenon. Some phenothiazines, especially in large dosage, may produce amenorrhea. ${ }^{60}$

Suzuki and associates ${ }^{293}$ gave chlorpromazine intramuscularly over 5 days (225 mg. total) to various gravid and nongravid and postclimacteric women and measured 
the urinary excretion of various hormones. Chlorpromazine caused a reduction in the urinary levels of gonadotropin, estrone, estradiol, estriol, pregnanediol, and total 17hydroxycorticoids. Total 17-ketosteroids was not effected in pregnant, toxemic, as well as postclimacteric women. The drug suppressed the excretion of gonadotropin in pregnant women, suggesting it inhibits the secretion of chorionic as well as pituitary gonadotropin. Chlorpromazine also inhibited the response of rabbit ovaries to gonadotropin in the Friedman test. The authors felt that the reduction in estrogen and progesterone levels was due to central factors as well as reduction of function of peripheral endocrine glands.

Hooper, Welch, and Shackelford, ${ }^{148}$ reported that 26 of 100 female psychiatric patients receiving various tranquilizers developed abnormal lactation. The drugs included chlorpromazine, proclorperazine, thioridazine, trifluoperazine, fluphenazine, meprobamate, and combinations of these. The incidence of abnormal lactation paralleled drug dosage. Abnormal lactation did not occur when antidepressants were combined with the tranquilizer. One false positive pregnancy test result was encountered in a patient taking 30 to $35 \mathrm{mg}$. of proclorperazine per day. Lactation has also been observed by others with reserpine and trimeprazine. Sulman ${ }^{292}$ used the nipple test in the male rat to determine the effects of chlorpromazine, reserpine, and other drugs for mammotropic activity which might serve as a prediction of lactogenic activity in man. He ascribed the lactogenic activity of chlorpromazine and reserpine as caused by hypothalamic depression.

Scott and Nading ${ }^{27 \pi}$ compared the relative effectiveness of a series of phenothiazines in causing release of melanocytestimulating hormone in intact and hypophysectomized frogs. All drugs tested in the hypophysectomized frogs were without effect. It was shown that all of the various phenothiazines tested were effective in intact frogs in releasing melanocyte-stimulating hormone. A striking correlation was evident between this research in frogs and the report of $A y d^{10}$ in which he compared the potency of the same agents in the treatment of psychiatric disorders in man. The relative ranking for the ten drugs was almost identical. Any significance of this hormone in man is of course unknown.

Brauchitsch $^{40}$ has reviewed the extensive literature on the effects of various tranquilizing drugs, including chlorpromazine and reserpine, on the endocrine system. He proposes that the first phase of action is an increase in adrenocortical activity and a decrease in thyroid activity. In the second phase, a reversal of endocrine equilibrium takes place depending upon dose, duration of treatment, etc. Thyroid activity gradually returns toward normal.

The interaction between chlorpromazine and antidiuretic hormone is not completely known. Moyer and co-workers ${ }^{231}$ reported that acute administration of chlorpromazine parenterally usually increases urinary flow in normal man and dogs independently of a change in renal hemodynamics. The drug appeared to increase sodium and water excretion. Parrish and Levine ${ }^{242}$ found that after intravenous administration of chlorpromazine ( $25 \mathrm{mg}$.) to various convalescent patients free of renal disease, the effective renal plasma flow and glomerular filtration rate rapidly decrease, with no change in the ratio of clearance of sodium or potassium to clearance of inulin. There was a concomitant increase in urine output and a decreased urinary excretion of an antidiuretic substance. They suggested this may be due to either a direct effect on the renal tubule or an indirect effect by way of central depression of antidiuretic hormone release. Cohen ${ }^{62}$ also was able to show that chlorpromazine infused intravenously at $3.5 \mathrm{mg}$. per hour was an effective diuretic agent in normal individuals and in patients with cor pulmonale in congestive failure. In normal subjects, the water diuresis was not accompanied by an electrolyte diuresis, but that in the patients with congestive failure was. Potassium excretion did not change. With the 
small doses of chlorpromazine used, the blood pressure did not change significantly. The glomerular filtration rate rose in most of the congestive heart patients but not in the normal persons.

The majority of reports suggest that chlorpromazine shows antidiuretic properties in hydrated rats. These include Meier, Brüni, and Tripod, ${ }^{221}$ Kovács and associates, ${ }^{188}$ Supek, Keckes, and Vojvodic, ${ }^{294},{ }^{295}$ and Tiwari, Jindal, and Jaiswal. ${ }^{306}$ On the other hand, Kivalo, Rinne, and Marjanen ${ }^{171}$ observed a diuretic action and Dasgupta ${ }^{70}$ no effect. Supek and colleagues ${ }^{294-296}$ noted that antidiuresis induced by nicotine injection or posterior pituitary extract was significantly prolonged by chlorpromazine. Tiwari, Jindal, and Jaiswal ${ }^{306}$ compared various phenothiazines as well as morphine, pentobarbital and reserpine on both waterinduced and saline-induced diuresis. Within 1 hour after injection, morphine, chlorpromazine, and promazine showed antidiuretic effects, while the ethyl diethylamino analogue showed a diuretic response. Promazine was less effective than chlorpromazine, while morphine was the most antidiuretic. Reserpine in very small doses had no significant effect. In saline-loaded rats, pentobarbital, proclorperazine, and dimethylaminoethyl phenothiazine were diuretic, while chlorpromazine and promazine were still antidiuretic.

Biochemical mechanism of action. The substituted phenothiazines are adrenergic, cholinergic, and histaminergic blocking agents. Inasmuch as the adrenergic blocking effects predominate, the central adrenergic blocking properties of the substituted phenothiazines have been tacitly assumed by many to account for their central actions. That this may not be the only mechanism is suggested by the numerous studies, especially in vitro, of the widespread biochemical effects of the phenothiazines. Unfortunately, most published reports are based upon data in vitro obtained at high concentrations (for in vivo) such as $10^{-4} \mathrm{M}$. Frequently, no appropriate physical controls (such as alteration of surface tension, mitochondrial swelling, etc.) have been made. As a result, much of the literature data must be taken lightly. The studies reviewed are presented only as possible leads to the biochemical actions of these agents and should not be accepted as definitive evidence of the mechanisms of action of the phenothiazines.

One of the first suggestions on the biochemical mechanisms of action of chlorpromazine was that it produced uncoupling of phosphorylation from oxidation. ${ }^{1}$ Quastel and Quastel ${ }^{259}$ reviewed some of the evidence that chlorpromazine acts as an uncoupler. They have pointed out that chlorpromazine has certain similarities to amobarbital and other barbiturates in this regard. Berger ${ }^{29}, 30$ demonstrated that chlorpromazine in concentrations of $2 \times$ $10^{-4} \mathrm{M}$ was effective in uncoupling phosphorylation from oxidation in rat liver mitochondria. The drug, however, under similar conditions failed to uncouple with brain mitochondria. Chlorpromazine appeared to act at all of the steps along the electron transport chain. Interestingly, brain mitochondria had a greater ability to absorb chlorpromazine than liver mitochondria. Dawkins, Judah, and Rees ${ }^{72}$ showed that in vitro concentrations of chlorpromazine of $1 \times 10^{-4} \mathrm{M}$ uncoupled the phosphorylation from oxidation of reduced cytochrome c. The drug did not affect the phosphorylations coupled to the prior steps in the electron transport chain. Chlorpromazine competitively inhibited cytochrome oxidase. These effects were found in both rat brain and liver mitochondria.

Bernsohn, Namajuska, and Cochrane ${ }^{31}$ compared chlorpromazine, promazine, chlorpromazine sulfoxide, and promethazine in ability to inhibit brain cytochrome oxidase and adenosine triphosphatase. In concentrations of $10^{-4} \mathrm{M}$, chlorpromazine was slightly more effective than promazine and promethazine; chlorpromazine sulfoxide had no significant inhibitory effect. The findings with chlorpromazine are in agreement with those of Abood. ${ }^{1}$

Helper and associates ${ }^{133}$ determined the 
effects in vitro of a large series of heterogeneous centrally active drugs on rat brain and liver succinoxidase activity. In sufficient concentration, all drugs inhibited activity; however, chlorpromazine was most effective, followed by promazine, promethazine, and reserpine. In all cases, cytochrome oxidase was more sensitive and succinic dehydrogenase less sensitive to inhibition than the complete succinoxidase system. Brain succinoxidase was more resistant to inhibition than liver succinoxidase. The authors did not feel that inhibition of this system was the mechanism of action of all the tranquilizers studied.

If chlorpromazine were a very effective uncoupling agent in vivo, one would expect the brain levels of high energy phosphates to decrease. The reverse is actually true, indicating that utilization is more depressed than synthesis. Grenell, Mendelson, and McElroy ${ }^{119}$ showed that chlorpromazine in large doses given to rats had no effect on the oxygen uptake or the in vitro rate of acetylation of sulfanilamide. It did, however, induce a marked increase in adenosine triphosphate levels, indicating a decrease in utilization. The greatest increase was in the midbrain, followed by cerebellum, cerebral cortex, and medulla. Subsequently, Weiner and Huls ${ }^{325}$ showed that chlorpromazine had no effect or elevated slightly the levels of adenosine triphosphate and creatine phosphate depending upon how the animals were sacrificed. Their results suggested that chlorpromazine depressed the utilization of brain adenosine triphosphate so that a given stimulus resulted in less use of labile phosphate. The biochemical effects in vitro of chlorpromazine, reported as interfering with oxidative enzyme systems and uncoupling of oxidative phosphorylation, therefore, appear unrelated to its primary action in vivo.

Depending upon the experimental conditions, chlorpromazine can decrease or increase the uptake of $\mathrm{P}^{32}$ inorganic phosphate into the brain phosphatides. Wase, Christensen, and Polley ${ }^{322}$ reported that 48 hours after administration of large doses of chlorpromazine to rats, there was a reduction in the incorporation of phosphate into the phosphatides of the hypothalamus and cortex. After 24 hours, the hypothalamus showed increased incorporation. In an in vitro study using guinea pig brain slices, Magee, Berry, and Rossiter ${ }^{213}$ found that chlorpromazine in concentrations of $10^{-1} \mathrm{M}$ stimulated the uptake of $\mathrm{P}^{32}$ into phosphatidyl serine, inositol phosphatidyl, and possibly phosphatidic acid. The labeling of phosphatidyl ethanolamine was unaffected, while that of lecithin was reduced. Ansell and Dohmen ${ }^{8}$ showed that chlorpromazine given in vivo to rats also caused a marked reduction of $\mathrm{P}^{32}$ incorporated into lecithin as well as phosphatidyl ethanolamine. Strickland and Noble ${ }^{290}$ studied the incorporation of $\mathrm{P}^{32}$ into different areas of the guinea pig brain following chlorpromazine both in vitro and in vivo. Chlorpromazine in concentrations of $10^{-4} \mathrm{M}$ had no effect on the incorporation of $\mathrm{P}^{32}$ into phosphatides of slices of liver, kidney, or pancreas but caused a definite increase in labeling in the brain. Brain homogenates and especially mitochondria took up the labeled phosphate mainly in phosphatidic acid, inositol phosphatide, and possibly phosphatidyl serine. No effect was noted on the incorporation of $\mathrm{P}^{32}$ by lecithin and phosphatidyl ethanolamine in contrast to the results of Magee, Berry and Rossiter. In vitro, all areas of the brain except the caudate nucleus and white matter of the cerebellum showed increased labeling after chlorpromazine in concentrations of $10^{-4} \mathrm{M}$. In vivo, chlorpromazine-treated cats showed twice as high uptakes of $\mathrm{P}^{32}$ into phosphatides in all areas of the brain studied.

In high concentrations in vitro, the phenothiazines inhibit oxygen consumption of rat brain slices and alter the distribution of glutamic acid and $\gamma$-aminobutyric acid between the brain slice and the incubation media. ${ }^{92}$ Although its significance is unknown, chlorpromazine in large doses to cats prevents the methylation of $\mathrm{C}^{14}$-labeled histamine by the brain. ${ }^{326}$ Cupric chloride has a similar effect. 
Chlorpromazine and methoxypromazine given in large doses to rats significantly increase brain glutamine levels in contrast to meprobamate and reserpine. A rise in brain alanine was also produced by the administration of these phenothiazines as well as by various convulsants. In contrast, phenobarbital produced a fall in both glutamic acid and $\gamma$-aminobutyric acid in the brain. Acetazolamide had a similar effect, but diphenylhydantoin did not affect either glutamic acid or $\gamma$-aminobutyric acid levels. ${ }^{76}$

The chemical reactivity of the phenothiazine molecule is also evidenced by various reports in the literature that chlorpromazine complexes flavine adenine nucleotide, chondroitin sulfate, heparin, and ti. a brain ganglioside strandin. The latter agent is distributed in the highest concentrations in the cerebral cortex. Little is known of the physiologic significance of strandin other than its inhibition of blood clotting and viral hemagglutination. Harris, Saifer, and Volk ${ }^{127}$ have suggested that the high concentrations of strandin in the cerebral cortex protect the cortex from depression by chlorpromazine and related phenothiazines.

Evidence is available that chlorpromazine has marked interactions with neurotransmitter substances producing effects other than simple block of receptor sites. For example, Mariani and Vertua ${ }^{216}$ have shown that chlorpromazine reduces the binding of serotonin by various tissues of the guinea pig both in vitro and in vivo. Pletscher and Gey $^{253}$ studied the effects of chlorpromazine on the precursors of serotonin and catecholamines. Chlorpromazine reduced the 5-hydroxytryptophan-induced rise of serotonin in the rat brain in vivo and in isolated brain. The drug also increased the blood:brain ratio of $\mathrm{C}^{14}$-labeled 5-hydroxytryptophan and $\mathrm{C}^{14}$-labeled 3:4-dihydroxyphenylalanine. Inasmuch as decarboxylation of aromatic amino acids was not inhibited by chlorpromazine in vivo, these investigators concluded that chlorpromazine reduced the uptake of aromatic amino acids in the brain. Chlorpromazine and chlorprothixene do not affect the content of endogenous rat brain serotonin, dopamine, and norepinephrine. ${ }^{109}$ However, these agents antagonized the release of brain amines by reserpine and their increase after iproniazid or 5-hydroxytryptophan. They suggested that the phenothiazines and related drugs act by decreasing the permeability of the storage granules for these brain amines. Guth and Spirtes ${ }^{123}$ have also suggested that chlorpromazine affects permeability. Furthermore, they have offered evidence of a selective accumulation of chlorpromazine in the hypothalamus and, in particular, in mitochondria. The release of acetylcholine from synaptic vesicles was inhibited by very small concentrations of chlorpromazine. This may account for the observation of Tatlow, Fisher, and Dobkin ${ }^{304}$ that chlorpromazine suppresses acetylcholine release from the cat's cortex as well as in cortical tissue removed at the time of lobotomy in schizophrenic patients.

Potentiation of the action of various drugs. The phenothiazines are known to potentiate the actions of various drugs such as the barbiturates, narcotics, and anesthetics. This effect is dose dependent, and in man in usual therapeutic doses, one may or may not obtain potentiation. Moyer ${ }^{230}$ felt that this potentiation was extremely variable. In some patients, there was a marked effect, while in others it was minimal. The potentiating actions of chlorpromazine and promethazine on the respiration of man have been recorded by Reckless $^{264}$ with a spirometer. Both phenothiazines potentiated the actions of thiopental, diethyl ether, tubocurarine, and narcotic analgesics including meperidine and morphine. Depending upon the type of neuromuscular blocking agent, potentiation or antagonism is observed as well as a direct blocking effect in very large doses. ${ }^{291}$

Fraser and Isbell ${ }^{101}$ showed that chlorpromazine ( $25 \mathrm{mg}$. intramuscularly or 50 mg. orally) increased the intensity of the miotic and sedative effects of 10 and 30 
mg. of morphine subcutaneously in postaddicts. Interestingly, the combination did not depress the respiratory minute volume any more than morphine alone. Reserpine (1 mg.) seemed to enhance the sedative effects of $30 \mathrm{mg}$. of morphine.

The interaction between phenothiazines, reserpine, meprobamate, and ethyl alcohol given to 4 young adults was studied by Burge. $^{54}$ Except for meprobamate, the drugs tested potentiated depressant effects of alcohol. Tipton and colleagues ${ }^{305}$ determined the effects of chlorpromazine, reserpine, atropine, and hexamethonium on alcohol blood levels in rabbits. After oral administration of ethyl alcohol, chlorpromazine alone enhanced the levels of alcohol in the blood. These investigators concluded that this effect was not due to an increase in absorption but was related to inhibition of alcohol metabolism. In fact, Khauw ${ }^{168}$ has shown that chlorpromazine in vitro inhibits alcohol dehydrogenase. Chlorpromazine potentiation in vivo in man of the effects of alcohol has been repeatedly observed. ${ }^{336}$

Several studies comparing the structure and potentiating activity of the phenothiazines have appeared. Dobkin ${ }^{80}$ compared various phenothiazine derivatives in their ability to prolong thiopental anesthesia in the dog. Unfortunately, different doses of the various drugs were used, so that it was impossible to compare them quantitatively. Nevertheless, in the doses used, promazine, propiomazine, and prothipendyl more than doubled the hypnotic effect of thiopental. Levomepromazine, imipramine, and methdilazine also caused a consistent prolongation of thiopental anesthesia. Mepazine, proclorperazine, and trifluoperazine had no effect on the duration of thiopental anesthesia.

Jindel, Tiwari, and Kherdikar ${ }^{157}$ compared the effects of equal doses (by weight) of a series of substituted phenothiazines and related drugs on pentobarbital sleeping time in mice. Chlorpromazine was quite effective, although its derivative lacking the dimethyl group on the terminal nitro- gen and position 2 chlorine was also very effective. Promethazine was less effective than chlorpromazine. Replacement of the phenothiazine ring with phenoxazine reduced potency as well. Of interest is the fact that those compounds which potentiated pentobarbital sleeping times also showed antidiuretic effects in saline-induced diuresis in rats. Those compounds which had no effect or reduced pentobarbital sleeping times had a diuretic effect against saline-induced diuresis. The authors suggest these findings may be related to the varying ability of the phenothiazines to release antidiuretic hormone. It is interesting also to note that others have shown the injection of antidiuretic hormone prolongs barbiturate sleeping time. Certainly, it seems most unlikely that antidiuretic hormone release is the only factor involved in phenothiazine potentiation of pentobarbital anesthesia, but it may be contributory. Further experiments obviously are necessary.

Metabolic fate. The extremely complex metabolic fates of the substituted phenothiazines are only partially known. Recently, a selected annotated reference list has been made available (by the Psychopharmacology Service Center ${ }^{313}$ ) which summarizes some of the hundreds of references available on metabolic studies and the analytic methods used for clinically useful phenothiazines. Even a casual survey of this literature provides one with a formidable impression of the complexity of the problem.

The phenothiazines are metabolically highly reactive. Animal studies are avail, able on the various metabolites of phenothiazine itself. These metabolites vary from species to species. Substitutions on the phenothiazine ring add further substrates which may be sites for metabolic alteration. Salzmann and Brodie $^{270}$ identified chlorpromazine sulfoxide as a metabolite of chlorpromazine in both the dog and human. In 2 patients taking $900 \mathrm{mg}$. of chlorpromazine orally a day, only negligible amounts of unchanged drug and about 5 
per cent as the sulfoxide appeared in the urine. In dogs, only 10 to 15 per cent of the chlorpromazine could be accounted for as the sulfoxide. The sulfoxide could possibly be further metabolized to the sulfone. Chlorpromazine sulfoxide was less sedative and produced less hypotension than the parent compound. These investigators noted that chlorpromazine was metabolized slowly, in part due to its high degree of localization in various tissues. Of particular significance was its high concentration in dog brain, reaching 70 times the level in plasma.

Although humans and dogs excrete somewhat similar urinary metabolites of chlorpromazine, there are some qualitative and quantitative differences. ${ }^{113}$ Urine samples from humans receiving $600 \mathrm{mg}$. of chlorpromazine a day and dogs receiving 8 mg. per kilogram a day orally were analyzed. Dogs excreted nine phenothiazines in moderate amounts and a tenth in traces. The compounds identified included the following, expressed as the percentage of the administered dose: desdimethyl chlorpromazine sulfoxide, 1.1 per cent; desmonomethyl chlorpromazine sulfoxide, 5.1 per cent; chlorpromazine sulfoxide, 5.1 per cent; and chlorpromazine or promazine, 2.8 per cent. Humans excreted nine compounds, which included the following, expressed as the percentage of the administered dose: desdimethyl chlorpromazine sulfoxide, 3.7 per cent; desmonomethyl chlorpromazine sulfoxide, 1.8 per cent; chlorpromazine sulfoxide, 0.4 per cent; and chlorpromazine or promazine, 6.1 per cent. Chlorpromazine sulfoxide concentrations were not large in either man or dog. $\mathrm{Hu}$ man urine contained two series of phenolic derivatives, the dog only one.

Huang, Sands, and Kurland ${ }^{150}$ found chlorpromazine glucuronides as the major urinary metabolites of psychotic patients given 100 to $400 \mathrm{mg}$. of chlorpromazine three times a day. Chlorpromazine sulfoxide and the hydroxy derivatives were present in smaller amounts. Unchanged chlorpromazine was found in very small quan- tities. Glucuronide formation appeared to be the most important pathway of chlorpromazine degradation. After discontinuation of the drug, the glucuronides were detectable in the urine for as long as 30 weeks. It seemed that those patients excreting the glucuronides for a long period regressed more slowly.

Forrest, Forrest, and Mason ${ }^{98}$ have reviewed the various urine tests for detecting phenothiazines and related substances. They pointed out that the partially oxidized, highly reactive intermediary drug metabolites of phenothiazines probably account for much more of the metabolism of the phenothiazines than sulfoxide formation. Other metabolites, partly free and combined glucuronic acid conjugates including oxidative changes in the nucleus, and demethylation of the side chain have been reported. During continuous drug administration to man, approximately half of the daily intake of chlorpromazine appears in the urine. Lesser amounts occur in the urine after single doses. The balance is excreted in the feces, and some resides in various body depots. Only 20 per cent of the amount of chlorpromazine contained in the urine is in the form of solvent extractable metabolites. The balance of 80 per cent of urinary drug is in the form of polar, intermediary oxidative metabolites of various types of hydroxylated derivatives. The absolute as well as the ratio of individual drug metabolites varies with each phenothiazine and to a lesser extent in different patients on the same phenothiazine. Forrest, Forrest, and Mason did not observe any differences in therapeutic efficacy and urine color tests for intramuscular or oral doses of chlorpromazine in the same patient. When spansules were used repeatedly, however, a 30 per cent increase in dose was necessary to obtain the same previous clinical effect and urinary drug level according to test I of Forrest. Forrest, Forrest, and Mason ${ }^{98}$ have summarized the qualitative urinary color tests for the rapid identification of various phenothiazines and related substances. Tests I and II are for 
chlorpromazine, promazine, and mepazine. The tests utilize $1 \mathrm{ml}$. of urine and $1 \mathrm{ml}$. of 20 parts 5 per cent ferric chloride and 80 parts 10 per cent sulfuric acid. Test III is for thioridazine. It consists of mixing 1 $\mathrm{ml}$. of urine and $1 \mathrm{ml}$. of a test solution containing 2 parts 5 per cent ferric chloride and 98 parts 30 per cent sulfuric acid. Test IV is for imipramine. It consists of mixing $0.5 \mathrm{ml}$. of urine with $1 \mathrm{ml}$. of a test solution containing 25 parts 0.2 per cent potassium dichromate, 25 parts 30 per cent sulfuric acid, 25 parts 20 per cent perchloric acid, and 25 parts 50 per cent nitric acid. Test $\mathrm{V}$ is for most phenothiazines such as chlorpromazine, promazine, triflupromazine, proclorperazine, trifluoperazine, perphenazine, fluphenazine, thiopropazate, acetophenazine, mepazine, thioridazine, promethazine, and others. This ferric, perchloric, and nitric acid (universal) test consists of mixing $1 \mathrm{ml}$. of urine and $1 \mathrm{ml}$. of test solution which consists of 5 per cent ferric chloric acid, 45 parts 20 per cent perchloric acid, and 50 parts 50 per cent nitric acid. In all tests, the color reaction must be read promptly and compared to a color chart in order to estimate the daily dose of phenothiazine ingested. Numerous ketonic and aldehydic substances and other drugs may interfere with these color tests; therefore, these must be kept constantly in mind. Some of the more obvious sources of false positives include (1) $p$-aminosalicylic acid in tuberculosis patients, (2) phenylketonurics, (3) patients on high doses of estrogens, and (4) patients with impaired liver function.

Forrest and Forrest ${ }^{97}$ have reported that urine samples of chronic mental patients who received daily doses of $600 \mathrm{mg}$. of chlorpromazine for a long time showed 5 per cent excretion as the sulfoxide, 0.75 per cent as unchanged chlorpromazine, and 0.5 per cent as thionium hydroxide. Discontinuation of the drug showed that unchanged chlorpromazine was excreted in the urine in trace amounts for several weeks, and thionium hydroxide for many weeks and months. Forrest and Forrest ${ }^{99}$ subsequently reported that after drug discontinuation, trace amounts may be detected in the urine for periods up to 6 months.

Huang and associates ${ }^{149,}{ }^{150}$ studied the urine of chronic schizophrenic patients given chlorpromazine in doses of 100 to $400 \mathrm{mg}$. three times daily for the presence of free chlorpromazine and its three sulfoxides, including chlorpromazine sulfoxide, and its demethylated analogues, nor-1-chlorpromazine sulfoxide and nor-2chlorpromazine sulfoxide. The average excretion of these substances was from 1.31 to 20.54 per cent of the administered dose. Of this, free chlorpromazine was less than 1 per cent, while the sulfoxides ranged from 1 to 18 per cent of the administered dose. After medication was stopped, free chlorpromazine and its sulfoxides disappeared from the urine in 5 days. Haynes ${ }^{132}$ analyzed the urinary excretory products during and after cessation of 200 to 800 mg. of chlorpromazine administered daily for periods of 4 to 11 days. The products which were ether soluble included free and bound chlorpromazine and its sulfoxide. After drug discontinuation, the urinary metabolites dropped significantly after the first day but continued to be present in small amounts for 6 days afterward.

Posner $^{257}$ suggests that on theoretic grounds, chlorpromazine can be metabolized to many different compounds. These include the sulfoxide, demethylated sulfoxides, $N$-oxide, phenolic derivatives, and conjugates of these with glucuronide. It is possible that some of these metabolites might contribute to the pharmacologic activity of the phenothiazines. To date, the sulfoxide derivative is known to be much less potent, but other metabolites might be more active. Posner has noted as many as fifteen different metabolites of chlorpromazine or promazine on paper chromatograms of human urine. He postulates some of these include modification of the side chain, including 3-hydroxy, 3,7-dihydroxy, the 3hydroxy sulfide, and the 3,7-dihydroxysulfide. 
Phenothiazines can apparently cross the human placenta. Behn, Fram, and Fretwurst ${ }^{22}$ reported that phenothiazine derivatives could be detected in the urine of newborn babies whose mothers had been given $50 \mathrm{mg}$. of chlorpromazine and promethazine within 30 minutes prior to delivery. About 0.5 to 1 per cent of the dose given the mother was in the child. Most of the excretion in the child occurs within the first 48 to 60 hours. The concentration of the drug in the fetus depends upon the time interval between administration to the mother and delivery. Marx ${ }^{218}$ has reviewed the literature on the placental transfer of many drugs, including the phenothiazines. Every known sedative, hypnotic, and anesthetic drug which passes the blood-brain barrier apparently also passes the placental barrier.

The pharmacologic effects of chlorpromazine apparently are the result of its presence in appropriate tissue sites. Christensen and Wase ${ }^{59}$ studied the distribution of $\mathrm{S}^{35}$-labeled chlorpromazine given intraperitoneally to mice. Radioactivity was rapidly and widely distributed, with the spleen and brain retaining the highest levels up to 5 days. Brain concentrations paralleled the degree of central nervous system depression. The maximal radioactivity occurred in the brain and kidney before any other tissue. Wase, Christensen, and Polley ${ }^{322}$ further studied the accumulation of $\mathrm{S}^{35}$-labeled chlorpromazine in the rat brain. The radioactivity was found in the lipid fraction, none in the protein fraction. In contrast, in the liver, about 5 to 10 per cent was protein bound. Using a chemical technique for converting chlorpromazine to the sulfoxide and measuring it spectrophotometrically, Wechsler and Roizin $^{324}$ were unable to find a high concentration of chlorpromazine in the rat brain. Assuming the results of both investigators to be valid, this would suggest that the $S^{35}$ label from chlorpromazine was exchanged for other tissue sulfur or that chlorpromazine in the brain is not easily extractable or converted to the sulfoxide.
Walkenstein and Seifter ${ }^{321}$ studied the tissue distribution of $\mathrm{S}^{35}$ promazine in dogs and rats. A large intraperitoneal dose of $\mathrm{S}^{35}$ promazine given to the dog was excreted about 70 to 80 per cent in the urine and 20 to 30 per cent in the feces in about 7 days as measured by the radioactivity. Rats were also given the drug orally and intraperitoneally. Fifteen minutes after injection, 50 per cent of the blood radioactivity was due to metabolically altered drug. No unchanged promazine was found in the blood after 24 hours. Highest concentrations were in the lung, liver, spleen, and kidney. Concentrations in the brain were low. This is in striking contrast to the data of Christensen and Wase on $\mathrm{S}^{35}$ labeled chlorpromazine in the mouse. ${ }^{59}$ In the urine, the promazine appeared unchanged as the monodemethylated compound and the corresponding sulfoxides. All of these compounds accounted for 10 per cent of the administered dose. Parenteral administration of promazine gave higher tissue levels than oral administration.

The Russian investigator Fyodorov ${ }^{105}$ has studied in detail the fate of $\mathrm{S}^{35}$-labeled chlorpromazine, promazine, and chlormepazine in various species of animals and man. In the dog, the highest concentration of radioactivity due to $\mathrm{S}^{35}$-labeled chlorpromazine was found in the lung, followed by the adrenal, liver, intestine, and brain. Only traces occurred in the blood. Radioactivity in the bile was high. There was a difference in the distribution of chlorpromazine depending on whether it was given intramuscularly or orally. Upon oral administration, a large amount of the chlorpromazine circulates in a closed enterohepatic route involving absorption from the gastrointestinal tract, portal transport to the liver, liver excretion via the bile, etc. As a result, less enters the systemic circulation than when it is given intramuscularly. Fyodorov suggests that this may indicate a greater incidence of liver toxicity with oral than with parenteral administration. Even after intramuscular ${ }^{\bullet}$ administration, some 
chlorpromazine is excreted via the bile. Species and the type of substituted phenothiazine also determine the tissue distribution of the drug. In man, $\mathrm{S}^{35}$ chlorpromazine given intramuscularly is excreted slowly. It disappears, however, from the blood very rapidly. Reasonable blood levels are present only 2 to 3 hours after injection. After 120 hours, only 16.3 per cent of the radioactivity appears in the urine, and after 192 hours, 20.2 per cent is excreted in the feces. Hollister, Traub, and Prusmack ${ }^{146}$ also report high biliary excretion levels of thioridazine given orally to schizophrenics.

Hetzel $^{137}$ reported that after $100 \mathrm{mg}$. of promazine given orally to 2 human subjects, serum levels of $0.5 \mu \mathrm{g}$ per milliliter were observed at 1 hour, A total of only 8.8 per cent of the dose was recovered in the urine after 24 hours. Oral administration of $25 \mathrm{mg}$. of promethazine to $2 \mathrm{hu}$ man subjects resulted in 7.5 and 10.4 per cent recovery of the drug in 24 hours.

Eiduson, Geller, and Cohen $^{90}$ have studied the excretion and metabolism of $S^{35}$ ring-labeled thioridazine in humans. Upon oral administration of 200 to $400 \mathrm{mg}$. of the drug, the serum levels rose to a peak in 2 hours and were maintained for 6 hours after ingestion. Detectable blood levels were observed even 96 hours later. Nevertheless, most of the phenothiazine disappeared rapidly from serum. Approximately 30 per cent of the original dose was excreted in the urine and 50 per cent in the feces. Over 60 per cent was found in the bile fluid of a patient with a bile duct cannula. The peak excretion in bile occurred 4 hours after ingestion of $200 \mathrm{mg}$. of the labeled compound. Serum was cleared most rapidly, with about 10 per cent of the original dose appearing in venous blood at 2 to 4 hours. The urinary chromatograms indicated at least twelve different metabolites of this compound in man.

An extremely interesting study on the role of drug metabolism in causing photosensitivity in two different species has been reported by Whitten and Filmer. ${ }^{330}$ When phenothiazine itself was administered to young cattle as an anthelmintic, photosensitized keratitis was observed. Similar keratitis was not observed in sheep maintained under the same circumstances. It was found that the sulfoxide of phenothiazine found in the blood of calves was also in high concentrations in the aqueous humor of the eyes. Sheep do not form the sulfoxide, and consequently, the aqueous humor does not contain it. These animals do not exhibit photosensitization, while young cattle do! Could some human patients showing photosensitivity to the substituted phenothiazines have a scheme of phenothiazine metabolism more like young cattle, while those not showing photosensitivity have one more like the sheep?

Physical dependence. Although some reports suggest that withdrawal of chronic phenothiazine therapy has no significant deleterious effect, ${ }^{269}$ the majority of reports are to the contrary (see study and review of the literature by Gross and colleagues $\left.^{121}\right)$. The question arises as to whether these exacerbations in illness following drug withdrawal are related to physical dependence to the phenothiazines. Boyd $^{37}$ has described withdrawal symptoms in rats chronically treated with chlorpromazine. In twin control experiments, young male albino rats were given increasing doses of chlorpromazine ( 1 to $200 \mathrm{mg}$. per kilogram) intramuscularly for 40 weeks. The animals developed tolerance to the sedative and lethal effects of chlorpromazine but no cross tolerance to pentobarbital. During chronic drug administration, the animals showed impairment of growth and fertility, although food and water intake and urine and fecal output were normal or greater than normal. The animals developed alkaluria and indicanuria, hypochronic anemia, and leukocytosis. No change in plasma lipids was observed. The abrupt withdrawal of chlorpromazine at the end of the fortieth week was followed by hyperkinesia, diarrhea, and occasionally death. Ten days after abrupt drug withdrawal, the animals began to grow and 
regain virility. The anemia and leukocytosis disappeared by the twenty-first day, and the animals were eating and excreting normally after about 40 days.

In man, the phenothiazines generally are not considered to produce any significant physical dependence and withdrawal symptoms. One exception is the report by Brooks. ${ }^{49}$ He noted a marked exacerbation of mental symptoms and muscular aches and pain, insomnia, and nausea upon abrupt drug withdrawal of a group of severely disturbed schizophrenic women who had been on prolonged periods ( 18 to 44 months) of chlorpromazine and reserpine. Thirteen of the 28 patients withdrawn were suffering symptoms similar to moderate withdrawal from morphine on the third day after the medication stopped. Another report suggesting possible withdrawal symptoms is that of Bennett and Kooi. ${ }^{26}$ These investigators compared the therapeutic effectiveness and toxicity of chlorpromazine, mepazine, perphenazine, proclorperazine, triflupromazine, and phenobarbital using a double blind procedure in 30 male schizophrenics. Prolonged heavy dosage of chlorpromazine, perphenazine, proclorperazine, and triflupromazine produced abnormal theta and delta changes in the electroencephalograph which correlated with drug-induced parkinsonism and clinical remission. No hepatic or hematologic toxicity was observed. After abrupt withdrawal of medication (by double blind substitution of a placebo capsule), there was a reaction delayed 4 to 6 days, after which 8 of the patients on various phenothiazines developed nausea, giddiness, vomiting, tremulousness, and delusions which were less elaborate than previously. The patients spontaneously reported that they suspected a change of medication. On resumption of the original medication, this symptomatology ceased within 3 days. These effects are hardly characteristic of other forms of drug withdrawal. The prolonged delayed reaction would suggest an exacerbation of the basic psychopathologic state except that its char- acter was modified. Electroencephalographic tracings during the drug withdrawal period did not show any evidence of major paroxysmal discharge, as has been observed with other drugs producing withdrawal symptoms such as the barbiturates, alcohol, meprobamate, or narcotics such as morphine. In monkeys, the administration of chlorpromazine subcutaneously in increasing doses to $2 \mathrm{mg}$. per kilogram, four times daily for a total of 45 days does not produce any obvious symptoms upon abrupt withdrawal. Some tolerance to the sedative effects was observed." A closely related congener of chlorpromazine, chlorprothixene, also does not produce signs of withdrawal after chronic administration of $5 \mathrm{mg}$. per kilogram subcutaneously per day for 3 weeks followed by $5 \mathrm{mg}$. per kilogram twice daily for 4 additional weeks in monkeys. Tolerance to the intensity and duration of the sedative effects also was observed. $\uparrow$ It would appear, therefore, that physical dependence on the substituted phenothiazines is not marked compared to other classes of depressant drugs.

Untoward effects and toxicity. The substituted phenothiazines produce numerous undesirable effects. ${ }^{63}, 95,114,140,174,205$ Some of these are due to their secondary pharmacologic actions: lethargy, drowsiness, tachycardia, hypothermia, dryness of the mouth, orthostatic hypotension, lactation, and extrapyramidal symptoms. Although it is not certain, jaundice, agranulocytosis, and skin reactions including photosensitivity are probably adverse effects related to drug allergy. $\mathrm{Ayd}^{11,12}$ has reviewed the untoward effects of the phenothiazines. He has pointed out that potency correlates best with the extrapyramidal reactions and inversely with sedation, undesirable autonomic effects, seizures, dermatitis, jaundice, and agranulocytosis. Autonomic nervous system effects are most prevalent with the weaker drugs such as promazine, mep-

\footnotetext{
*D. MeCarthy and G. Deneau: Personal communication, 1962 .

fG. Deneau: Personal communication, 1962.
} 
azine, and methoxypromazine. They are much less frequent with the potent agents such as trifluoperazine and fluphenazine. The weaker drugs also cause more endocrine disturbances.

Allergic reactions to the substituted phenothiazines usually occur in the first few months of treatment. These include various forms of dermatitis, jaundice, ${ }^{85}$ and agranulocytosis. Most blood dyscrasias occur within the first few months of therapy. Korst $^{187}$ has reviewed this subject in detail. Agranulocytosis appears to be a definitely established reaction to chlorpromazine and promazine; there are reports that the other phenothiazines may have this action. If it is recognized and the drug stopped, the patient will recover completely. He should be treated with antibiotics but probably not corticosteroids. The reaction appears to be due to individual idiosyncrasy. Cross sensitivity to other phenothiazines may occur. Recovery usually is complete if managed properly without residual bone marrow abnormality. ${ }^{20}$ More recently, Kähler ${ }^{162}$ determined the incidence and mortality rate of patients with agranulocytosis caused by the phenothiazines. The incidence of agranulocytosis was 0.07 to 0.7 per cent of the total number of patients treated with various phenothiazines, with a mortality rate as high as 40 per cent.

In an effort to determine the possible mechanisms of chlorpromazine-induced agranulocytosis, Pisciotta, Kohldahl, and Mally ${ }^{250}$ studied its effects on the uptake of $\mathrm{H}^{3}$-thymidine by granulocytes. Chlorpromazine inhibited the uptake of thymidine into granulocytes of normal cells but had no effect on its uptake by normoblasts when incubated with human bone marrow in vitro with $\mathrm{H}^{3}$-thymidine or uridine.

The untoward effects of the phenothiazines and other tranquilizing agents in children are similar to those in adults. Shaw ${ }^{280}$ has reviewed these in detail. The substituted phenothiazines are most commonly used in children for the symptomatic relief of nausea and vomiting. The most common adverse effects are extrapyramidal symptoms. These may be confused with encephalitis, etc. The original dosage for children has been arrived at on a proportional basis compared to the adult dose. Shaw suggests that children may have a greater sensitivity to these agents than adults, which might account for the numerous reports of extrapyramidal symptoms. Proclorperazine has been especially implicated in producing these effects. Pediatric patients with acute infections and severe dehydration may be extraordinarily sensitive. Treatment consists largely of cessation of the phenothiazine medication, sedation with phenobarbital, and the administration of antiparkinson drugs or caffeine. Sometimes extrapyramidal symptoms are protracted. The less common complications of phenothiazine therapy in children include jaundice, granulocytopenia, cutaneous eruptions, and hyperpyrexia. Chicoine $^{58}$ reported on 25 cases of serious drug reactions to various phenothiazines in children. The signs of intoxication included agranulocytosis, jaundice, extrapyramidal symptoms, somnolence, and semicoma which lasted for 8 hours to 4 days.

Kelsey and associates* observed three types of pathologic liver conditions associated with chlorpromazine. The most frequent type was a diffuse inflammatory change associated with biliary stasis. The clinical picture was an obstructive type of jaundice with a moderate elevation of alkaline phosphatase. None of the liver function tests associated with parenchymal liver damage were abnormal. The next type resembled acute hepatitis with clinical evidence of parenchymal liver damage as indicated by elevated thymol turbidity and prothrombin deficiency. In the most uncommon type, early cirrhosis was observed. The patients showed marked azotemia and a clinical picture of severe and prolonged chronic liver disease. Danhof ${ }^{68}$ has reviewed some of the hypothesized mechanisms for chlorpromazine-induced jaundice and studied in rats the role of various fac-

${ }^{\star}$ Quoted by Moyer. ${ }^{230}$ 
tors which might lead to liver disease. Chlorpromazine-treated animals maintained on a choline-deficient diet failed to show diffuse fatty metamorphosis. No eosinophilic infiltration or biliary canalicular plugging was evident. Chlorpromazinetreated rats, however, showed an increase in bile viscosity and reduced bile volume. These effects appeared to be related to the decreased water intake of the animal. The author stressed the fact that no signs of jaundice could be obtained in these animals. Increased biliary pressures have been reported by Menguy and associates ${ }^{223}$ in the cholecystectomized dog given chlorpromazine. An interesting attempt to prevent jaundice resulting from chlorpromazine in man was reported by Dreyfuss. ${ }^{86}$ It was based on the hypothesis that inspissation of the bile was the mechanism of the jaundice. Patients treated with chlorpromazine were given large amounts of fluid. In the first 362 patients treated without extra fluids, the incidence of jaundice was 4.4 per cent. After giving about $2.3 \mathrm{~L}$. of additional sweetened tea or water to each of another 588 patients, the incidence of jaundice dropped to 0.34 per cent. Both groups of patients were otherwise similarly treated.

Read, Harrison, and Sherlock ${ }^{263}$ described the case histories of 4 women who developed obstructive jaundice after 1 to 4 weeks on $75 \mathrm{mg}$. per day of chlorpromazine. Very high serum cholesterol levels and xanthomatous cutaneous deposits were noted in these patients. Clinical recovery was achieved in all cases, but serum cholesterol and phosphatase levels did not return even 7 to 18 months later. The authors referred to the relationship of their findings to primary biliary cirrhosis. Trifluoperazine has also been reported ${ }^{179}$ to cause cholestatic hepatitis with clinical, laboratory, and biopsy findings identical to those seen especially with chlorpromazine but also with promazine, mepazine, proclorperazine, and other phenothiazines.

Convulsions may be precipitated by any of the phenothiazines. They can occur in both epileptics and nonepileptics. The less potent phenothiazines such as promazine and mepazine especially can produce convulsions. In spite of this tendency to precipitate seizures, the phenothiazines can and should be used to control severe anxiety in the epileptic patient. For example, emotionally disturbed epileptics showed an excellent response to chlorpromazine. The drug was tolerated without marked alteration in seizure frequency in most patients as long as anticonvulsant medication was maintained. ${ }^{36}$ On chronic administration, the potentiation of barbiturate-induced drowsiness was negligible. The seizurelowering property of chlorpromazine has been studied in various patients subjected to electroshock therapy or chemically induced convulsions. Interestingly, in therapeutic doses, this effect is not obvious. For example, single oral doses of 150 to 200 $\mathrm{mg}$. of chlorpromazine 3 hours prior to electroshock therapy in psychiatric patients did not alter the course of the electroshock seizure, etc. Longer periods of sleep and occasional posttreatment hypotension were noted. ${ }^{34}$

The electroencephalographic or motor threshold for stroboscopic light flashes during a timed infusion of a convulsant hexazole $^{*}$ was not altered by increasing oral doses of chlorpromazine (150 to $800 \mathrm{mg}$. per day), reserpine ( 3 to $4 \mathrm{mg}$. per day) given chronically, or $100 \mathrm{mg}$. of azacyclonol given intravenously every 6 hours for eight doses in schizophrenic patients. ${ }^{287}$ In addition to lowering seizure threshold, chlorpromazine has also been reported to elevate it. For example, it has been used in the treatment of status epilepticus. ${ }^{71}$.

The extrapyramidal symptoms induced by the phenothiazines can frequently be quite dramatic. Ayd ${ }^{11}$ observed approximately a 39 per cent incidence of extrapyramidal reaction in 3,775 patients treated for 3 months to 6 years with various phenothiazines. Akathisia (motor restlessness) occurred in approximately 21 per cent,

*Fazole. 
parkinsonism in 15 per cent, and dyskinesia (dystonic reactions) in 2 per cent of the cases. A correlation was found between the percentage occurrence of these reactions and milligram potency of the drugs as follows: chlorpromazine, 35 per cent; triflupromazine, 36 per cent; proclorperazine, 45 per cent; thiopropazate, 44 per cent; perphenazine, 36 per cent; trifluoperazine, 60 per cent; and fluphenazine, 52 per cent. Some interesting sex differences were observed. Akathisia and parkinsonism occurred twice as often in women as in men. In men, dyskinesia was twice as common as in women. Dyskinesia occurred earliest ( 90 per cent within $4 \frac{1}{2}$ days), akathisia next, and parkinsonism last. Dyskinetic symptoms presented bizarre neuromuscular manifestations and could be mistaken for seizures, tetanus, meningitis, encephalitis, and poliomyelitis. These symptoms are promptly relieved by various antiparkinsonian drugs, including parenteral injections of 1 to $2 \mathrm{mg}$. of benztropine, biperiden, etc., as well as by diphenhydramine and dihydroxyphenylalanine ${ }^{208}$ and by caffeine. ${ }^{116}$

Several authors have emphasized that the motor symptoms induced by phenothiazines can mimic seizures or other serious diseases of the central nervous system. Shaw and colleagues ${ }^{281}$ reported a series of cases, including children and a few adults, who showed severe extrapyramidal symptoms (which they called seizures) frequently misdiagnosed for convulsions, poliomyelitis, infectious meningitis, tetanus, etc., after the use of various phenothiazines. Sometimes, these symptoms appeared within hours after small therapeutic doses. Scime and Tallant ${ }^{276}$ have reported that various extrapyramidal symptoms which may be mistaken for tetanus may occur with proclorperazine. They have emphasized that these can occur with small therapeutic doses. The duration of the reactions may vary from $1 / 2$ to 48 hours. To control mild reactions, phenobarbital was used. With severe reactions, amobarbital, phenobarbital, and benztropine were used to con- trol hyperactivity. No permanent aftereffects were noted in 8 patients having untoward extrapyramidal effects from proclorperazine.

Thioridazine appears to produce significantly fewer untoward effects such as extrapyramidal symptoms, lethargy and drowsiness, orthostatic hypotension, convulsions, and photosensitivity than other phenothiazines. ${ }^{169}$

Dobkin and $\mathrm{Palko}^{82}$ compared various phenothiazines at different doses intravenously as to their ability to depress salivary secretions induced by intravenous carbaminoylcholine and epinephrine. Levomepromazine, trifluoperazine, proclorperazine, methdilazine, and prothipendyl reduced salivary secretions by 50 per cent in doses which produced drowsiness and a dry mouth. Promazine in the dose used had negligible effects on induced salivary flow.

Patients who ingest an excess of chlorpromazine present two different clinical pictures. The first is related to extreme somnolence. With prodding, the patient will arouse but promptly fall back into a deep sleep. In such a patient, slight hypotension and respiratory depression may occur, but they are not a problem. The second clinical picture is one of hypotension. This may be a mild to moderate fall in blood pressure whether the patient is conscious or not. The skin may be markedly gray but warm and dry. The nailbeds usually are still pink, and the pulse is strong but more rapid than normal. Respiration is usually slow and regular. Hypotension may be very severe; in this case, the patient may present symptoms of shock including weakness, cyanosis, perspiration, and a rapid thready pulse. Treatment in each case is symptomatic. Inasmuch as chlorpromazine is an alpha adrenergic blocker, larger than usual doses of norepinephrine may be necessary to maintain the blood pressure. Other phenothiazines may present similar clinical pictures. As the potency of the compounds increases, there is a tendency for extrapyramidal symptoms to be present as part of the picture of acute 
overdosage. For example, overdosage with trifluoperazine (140 mg. total dose) with suicidal intent can be manifested as generalized muscle spasms and stiffness of the legs, back, and neck, increased patellar reflexes, and torticollis with consciousness present. ${ }^{14}$

\section{Rauwolfia alkaloids and related compounds}

Although preparations of the Rauwolfia alkaloids have been used for centuries in India for many illnesses, their widespread use in Western medicine began only after the isolation of reserpine from Rauwolfia serpentina by Mueller, Schlittler, and Bein $^{233}$ in 1952. Since then, numerous alkaloids have been isolated, some of which have been found therapeutically useful. With the exception of thebaine and papaverine, all of the Rauwolfia alkaloids are indole bases. ${ }^{274}$

Some, such as yohimbine, have been well known for a long time, whereas others are relatively new; still others are yet to be identified. The chemistry of these alkaloids is very complex, which perhaps is Nature's way of providing hints of their complex pharmacology (see Bein $^{23}$ ).

Among the compounds frequently used therapeutically or of special theoretic interest are those presented here.
Structure-activity relationships. A great deal of research activity has been devoted to the structure-activity relationship of the Rauwolfia alkaloids. In spite of the fact that many alkaloids have been isolated, reserpine still appears to be the most effective. Reserpine is a whitish crystalline powder which is sparingly soluble in water. It dissolves readily in organic acids such as acetic and citric and can be diluted in this way. Lyophilized reserpine phosphate is directly soluble in water.

On alkaline hydrolysis, reserpine yields reserpic acid, 3,4,5-trimethoxybenzoic acid, and methanol. Hydrolysis destroys pharmacologic activity. The compound can be reconstituted from reserpic acid by methylation to methyl reserpate and subsequent trimethoxybenzoylation. Early reports suggested that methyl reserpate was active, but this has not been confirmed. Changes in the esterifying acids in position 18 (replacing 3,4,5-trimethoxybenzoic acid) generally reduce activity. Minor alterations in the benzoic acid ester, however, result in compounds with somewhat similar activity. Rescinnamine is somewhat less potent centrally as well as peripherally than reserpine. Syrosingopine also is less potent but has a more selective peripheral action than reserpine. Removal of the methoxy group in ring $A$ of reserpine results in 11-desmeth-

A. Tertiary indole Rauwolfia alkaloids (naturally occurring and semisynthetic)<smiles>COc1ccc2c3c([nH]c2c1)C1CC2C(CC(Oc4cc(OC)c(OC)c(OC)c4)C(OC)C2C(C)=O)CN1CC3</smiles>

Reserpine 
<smiles></smiles>

Deserpidine<smiles>COC(=O)C1C2Cc3c([nH]c4cc(OC)ccc34)C3CCC(CC(OC=Cc4cc(OC)c(OC)c(OC)c4)C1OC)C2C3</smiles>

Rescinnamine<smiles>CCOc1ccc2c3c([nH]c2c1)C1CC2C(CC(OC)C(OC)C2C(=O)OC)CN1CC3</smiles>

Syrosingopine<smiles></smiles> 
B. Synthetic derivatives<smiles>COc1cc2c(cc1OC)C1CC(=O)C(C(C)C(C)C)CN1CC2</smiles>

Tetrabenazine<smiles></smiles>

Ro 4-1398<smiles>CCC1(O)CC2c3cc(OC)c(OC)cc3CCN2CC1C(C)C</smiles>

Ro $4-1284$<smiles>CCOc1cc2c(cc1OC)C1CC(OC(C)=O)C(C(=O)N(CC)CC)CN1CC2</smiles>

Benzquinamide<smiles>CCOc1cc2c(cc1OC)C1CC(O)C(C(=O)N(CC)CC)CN1CC2</smiles>

P-2565 oxyreserpine or deserpidine. This compound has qualitative and quantitative pharmacologic actions identical to reserpine. Interestingly, a shift of the position 11 methoxy group on ring $A$ of reserpine to position 10 (methoserpidine) has been claimed to produce a compound with a more selective peripheral action.

Methoserpidine, the isomeride of reserpine, has been reported by Gros, Peterfalui, and Jequier ${ }^{120}$ to have a hypotensive effect in man while lacking a central de- pressant action. This dissociation of central and peripheral actions has also been reported in mice, rats, and dogs. In cats, the agent produces sedation and depletion of norepinephrine from sympathetic ganglia as well as the hypothalamus. ${ }^{271}$ In contrast, in the rabbit, there is evidence for selective depletion of norepinephrine in sympathetic ganglia by this compound. Inasmuch as dogs show no sedative effect from this agent, it would appear that man behaves more like the dog and rabbit than the cat. 
A similar dissociation of peripheral and central depletion of norepinephrine and serotonin has been reported for syrosingopine in the rabbit and dog by Orlans, Finger, and Brodie. ${ }^{238}$ Doses of syrosingopine which do not sedate these species have peripheral cardiovascular hypotensive effects. Similar differential effects have been observed with small doses of reserpine by Carlsson and co-workers, ${ }^{56}$ but over a narrower dose range than syrosingopine. In man, syrosingopine can lower blood pressure in doses relatively free of central effects. ${ }^{63}$

An interesting chemical development is the synthesis of reserpine analogues, of which tetrabenazine is the best known. The compound is much less potent and shorter acting than reserpine. Although its therapeutic use appears quite limited, it is of considerable theoretic importance and serves as a prototype of many newer derivatives. Quinn, Shore, and Brodie ${ }^{260}$ showed in rabbits that the drug has a more selective effect centrally. The agent appears to depress brain norepinephrine and serotonin, having little effect peripherally. The agent appears to depress brain norepinephrine more than serotonin levels, although the sedative effect seems to be correlated with the latter. After intravenous administration, the compound is rapidly and almost completely metabolized. In contrast to reserpine, the duration of action of tetrabenazine is limited to the short time appreciable quantities are in the body. Inasmuch as animals given tetrabenazine before reserpine act as though they received only tetrabenazine, Quinn, Shore, and Brodie concluded that both drugs compete for the same receptor sites. In man, the drug is said to produce no diarrhea, in contrast to reserpine. ${ }^{317}$ Both compounds are said to have similar central nervous system effects, although undesirable central effects of tetrabenazine limit its clinical usefulness. Several other benzoquinolizines are of theoretic interest. Pletscher and associates $^{251,252}$ determined the effects of RO 4-1284 and RO 4-1398 on the brain levels of serotonin and norepinephrine in mice. They found that both compounds depressed brain serotonin equally but had differential effects on brain norepinephrine. Brain serotonin was maximally depressed at 1 hour and recovered in about 6 hours after each compound. On the other hand, brain norepinephrine was much more depressed by Ro 4-1284 than after Ro 4-1398; similarly, the degree of overt depression was greater after Ro 4-1284 than Ro 4-1398. They concluded that the sedative effects induced by these agents correlated better with the depression of brain norepinephrine rather than serotonin. Quinn, Shore, and Brodie, ${ }^{260}$ however, were not able to confirm this in mice as well as rabbits and concluded instead that the sedative effects more easily correlate with depression of brain serotonin than of norepinephrine.

Finger, Weisman, and Tretter ${ }^{96}$ have pointed out that there may be no correlation between depression of conditioned behavior and levels of the central amines, serotonin, and norepinephrine with several other substituted benzoquinolizines which they studied. One compound, P-2647 (benzquinamide), was much more effective in depressing avoidance behavior and produced negligible changes in the levels of brain amines as well as any overt sedation, ptosis, lacrimation, and salivation. On the other hand, its hydroxy derivative, P-2565, was less effective in depressing avoidance behavior and yet had greater effects in lowering brain amine levels. This compound produced overt sedation, ptosis, etc. If these results are confirmed, it would appear that ability to depress avoidance behavior and depletion of serotonin and norepinephrine are unrelated phenomena. Some of these interesting compounds are currently being evaluated in man as potential therapeutic agents. The relatively quicker onset and shorter duration of action makes them more desirable in some respects than reserpine. Furthermore, the possibility that a central effect (depression of conditioned behavior) may occur without any appreciable depletion of brain 
amines suggests that the latter may be more related to untoward effects than to the primary pharmacologic action of the drug. Only more research, both basic as well as clinical, will provide the necessary answers.

Central and autonomic nervous system effects. The marked behavioral alterations produced by reserpine and related compounds include mild depression of the central nervous system, a decrease in sympathetic activity and an increase in parasympathetic effects. In animals, the behavioral alterations may be dramatic. Hostile and aggressive monkeys become tame and can be safely handled. Conditioned avoidance and conditioned suppression behavior are depressed in all animal species tested. Human subjects receiving reserpine are more relaxed and tend to go to sleep more readily but are easily aroused even though large doses were ingested. The autonomic effects are clear-cut, with parasympathetic symptoms predominating. Thus, the pupils are miotic, pulse rate is decreased, blood pressure is lowered, gastrointestinal activity( including secretion of gastric hydrochloric acid) increases, and temperature regulation is altered. The fall in blood pressure and heart rate occur even though serum sodium and chloride levels are unchanged. In fact, a slight increase in the levels of these ions may occur. There is an extensive literature on the effectiveness of these agents in reducing anxiety and maniacal states. Kline ${ }^{175,176}$ reviewed some of the early reports of the use of the Rauwolfia alkaloids in psychiatric states and reported his own favorable results with it. The quieting effect on noisy wards was obvious, although the drug did not alter the basic schizophrenic process itself. Within a 1 year period, two major symposia appeared on the behavioral and pharmacologic effects of these compounds. $^{225,226}$ In the intervening years, clinical psychiatric interest in reserpine-like drugs has tended to decline as a result in part of the widespread use of the substituted phenothiazines. ${ }^{41}$ Nevertheless, the remarkable pharmacologic effects of the Rauwolfia alkaloids will always be of value in the selected psychiatric or hypertensive patient.

Reserpine on psychologic tests in man does appear to possess certain unique properties. Lehmann and $\mathrm{Csank}^{200}$ compared the effects of single oral doses of reserpine ( $2 \mathrm{mg}$.), chlorpromazine (100 to $150 \mathrm{mg}$.), proclorperazine ( 30 to $60 \mathrm{mg}$.), secobarbital ( 100 to $200 \mathrm{mg}$.), D-amphetamine ( 12.5 to $15 \mathrm{mg}$.), and caffeine citrate (600 to $900 \mathrm{mg}$.) on various psychologic tests in healthy human volunteers. Nine different psychologic tests were used, including visual function, tapping speed, reaction time, hand steadiness, learning capacity, and recall. Reserpine significantly increased afterimage sensitivity in contrast to chlorpromazine, proclorperazine, and secobarbital, which significantly decreased this function. Chlorpromazine and secobarbital also caused a significant decrease of flicker fusion. Proclorperazine also depressed afterimage sensitivity, but unexpectedly, it was a more powerful stimulant of psychomotor performance than D-amphetamine or caffeine. It caused a marked decrease in reaction time and significant improvement in hand steadiness, digit span, digit symbol, and cancellation tests. Lehmann and Csank pointed out that each drug has its own spectrum of psychologic activity. The critical flicker fusion and afterimage disappearance level tests were especially sensitive to the various drugs tested as well as to other clasies of psychotropic drugs such as the hallucinogens, including lysergic acid diethylamide and mescaline, which raised the critical flicker fusion frequency and the afterimage disappearance level. Another psychologic study in which reserpine and chlorpromazine were compared with somewhat similar results was that by Watt and Crookes. ${ }^{323}$ Reserpine in doses of 2 to $30 \mathrm{mg}$. per day to manic females caused an impairment in visual input as measured by the flicker fusion threshold, hue discrimination, and duration of a negative afterimage. There 
was no consistent effect on tests of motor or association functions. Chlorpromazine, in contrast, in doses of $300 \mathrm{mg}$. per day impaired both the flicker fusion and motor performance.

Depending upon the dose and duration of therapy, reserpine may show negligible or definite electroencephalographic changes. $^{75}$ Monroe and colleagues ${ }^{229}$ compared the effects of reserpine, chlorpromazine, and amobarbital on various cortical and subcortical sites in psychiatric patients with chronic implanted brain electrodes. Reserpine in doses of 5 to $10 \mathrm{mg}$. intravenously produced relaxation within 15 minutes after injection. The patients were quiet and appeared to be asleep. If they were approached, they would easily arouse. Sometimes they would say they were sleepy, but often they would not. The electroencephalographic recordings were not markedly altered. Combinations of reserpine and amobarbital produced deeper behavioral depression than either drug alone, although the electroencephalographic changes resembled those after amobarbital alone. Like reserpine, chlorpromazine in doses of 50 to $100 \mathrm{mg}$. given intravenously to 4 human subjects with implanted electrodes produced no distinctive electroencephalographic changes, except that the patients showed a more relaxed record with short bursts of drowsiness and occasional light sleep, during which the subjects appeared to be sleeping. Electroencephalographic and behavorial arousal was easily produced. The electroencephalographic effects which occur after chronic administration of chlorpromazine and other phenothiazines were also seen with chronic administration of reserpine in schizophrenics. ${ }^{142}$ Generalized dysrhythmia and focal slowing were most commonly observed.

The cardiovascular effects of reserpine in man have been described in detail in the first major symposium on this subject. ${ }^{225}$ In general, the hypotensive actions of this and related Rauwolfia alkaloids are mild, consisting of a slight but definite decrease of systolic as well as diastolic blood pressure. Intravenous infusions of 40 to $80 \mu \mathrm{g}$ per kilogram of reserpine in hypertensive patients reduced the mean arterial blood pressure within 4 hours. The drop in pressure was usually accompanied by nasal congestion, bradycardia, postural hypotension, and a feeling of relaxation. In the majority of patients, the blood pressure increased within 24 hours, but in a few, the blood pressure remained at normotensive levels for 2 to 3 days after the infusion. Even in such relatively large doses as $5 \mathrm{mg}$. intramuscularly, reserpine usually produces a decrease in mean arterial pressure within 20 minutes. Very large doses of $10 \mathrm{mg}$. produce an initial increase in blood pressure in some psychotic patients. It is not known to what extent the vehicle used for reserpine contributes to this response."

The initial hypertensive phase is especially prominent with large toxic doses of reserpine in the dog. ${ }^{84}$ Presumably, this is related to massive catecholamine release. In man in the usual therapeutic doses of reserpine, an initial pressor response is not seen because of compensatory mechanisms sufficient to handle the released catecholamines.

Winsor ${ }^{332}$ studied the pharmacologic effects of reserpine on various tests in man. In 5 patients studied before and 1 month after reserpine administered daily in doses of 0.25 to $0.50 \mathrm{mg}$, the galvanic skin response to inspiration was not significantly inhibited. Vasoconstriction of the digit to inspiration or pain induced by an electric shock, however, was definitely reduced, especially with oral doses of $2.8 \mathrm{mg}$. for as little as 3 days. Small doses of reserpine ( 0.25 to $0.50 \mathrm{mg}$.) daily for 30 days failed to inhibit the blood pressure or pulse rate increase resulting from placing the hand in ice water (cold pressor test). Larger doses were not apparently tried. This would be particularly important since one would expect the response to be depressed with adequate peripheral adrenergic nerve catechol-

*J. C. Saunders: Personal communication, 1956. 
amine depletion (see section on mechanism of action).

Endocrine effects. Gaunt and colleagues $^{108}$ studied the effects of reserpine on the endocrine system of the rat. Numerous effects were found which were related to stress such as the release of ACTH and possibly antidiuretic hormone. In the intervening years, evidence has accumulated that reserpine may inhibit estrus and menstruation, ${ }^{74}$ modify the uterine decidual response $^{\tau_{3}}$ altering fertility and block the release of pituitary gonadotropin. ${ }^{16,17}$ ACTH release may be stimulated or depressed and lactogenic hormone release enhanced (see Gaunt, Chart, and Renzi ${ }^{107}$ ).

Egdahl, Richards, and Hume ${ }^{88}$ have noted that acute administration of large doses of reserpine (5 mg.) intravenously to dogs caused a marked increase in adrenal 17-hydroxycorticosteroid output. The response was delayed, with a peak occurring between $1 / 2$ and 3 hours after drug injection. These effects are compatible with large intravenous doses of ACTH, though comparatively much delayed. Harwood and Mason ${ }^{128}$ compared the acute effects (zero to 4 hours) of varying doses of reserpine, chlorpromazine, and pentobarbital on the plasma levels of 17-hydroxycorticosteroids in the monkey. Reserpine in small doses caused an elevation in 17-hydroxycorticosteroids at 1 and 2 hours after injection which tended to return toward control levels at 4 hours. Large doses of reserpine caused an increasing elevation of plasma levels of these steroids to 4 hours. In low doses, chlorpromazine caused an elevation in plasma levels of 17-hydroxycorticosteroids, while in large doses it produced a fall. Reserpine was more potent than chlorpromazine in elevating the plasma levels of 17-hydroxycorticosteroids. In anesthetizing doses, pentobarbital depressed plasma levels of these steroids. Using the 17-hydroxycorticosteroid response to ACTH as a reference, it appeared that reserpine produced the maximal stimulation of the adrenal cortex. It is of interest that Tui, Riley, and Orr, ${ }^{310}$ using schizophrenic patients, also found an elevation in the 17-hydroxycorticosteroid levels in response to reserpine and a depression in response to chlorpromazine. Brodie and colleagues ${ }^{43}$ studied the actions of single doses of reserpine given to rats on various indices of pituitary-adrenocortical activity. Reserpine in doses which produced profound sedation and depletion of brain amines caused a decrease in adrenal ascorbic acid and elevated levels of plasma corticosterone and liver tryptophan peroxidase. Plasma free fatty acids at first diminished, then increased. Adrenal weight increased 40 per cent in 24 hours. The pharmacologically inert stereoisomers of reserpine had no effect upon the brain amines or pituitaryadrenal activity. The endocrinologic alterations induced by reserpine did not occur in hypophysectomized or adrenalectomized animals even though sedation and depletion of brain amines were observed. These findings correlate with previous reports that ACTH levels in the pituitary are reduced after reserpine.

Croxatto and Barnafi showed that reserpine administered chronically to normal rats decreased urine volume and sodium output. Paradoxically, the blood of these rats showed very little antidiuretic activity. Reserpine administration to rats given a water load produced an immediate antidiuretic effect. Similar findings have been reported by Gaunt and co-workers ${ }^{108}$ and Meier, Brüni, and Tripod. ${ }^{21}$

Mechanisms of action. The ability of reserpine and its related sedative alkaloids and synthetic derivatives to release various amines from the brain and other peripheral sites has resulted in considerable speculation that this accounts for its mechanism of

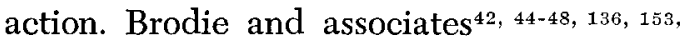
254, 255, 286, 288 have emphasized the importance of serotonin release, while Holtzbaur and Vogt, ${ }^{147,}{ }^{234}$ Paasonen and Krayer, ${ }^{239}$ Carlsson and colleagues, ${ }^{56}$ and others have emphasized depletion of catecholamines, including dopamine, norepinephrine, and epinephrine. Waalkes and Weissbach ${ }^{319}$ have shown that reserpine releases hista- 
mine in addition to serotonin from rabbit platelets in vivo. The rat mast cell apparently acts differently, for Bhattacharya and Lewis $^{33}$ have shown that reserpine does not disrupt mast cells and specifically releases their serotonin without affecting histamine content. In contrast, the compound 48/80 disrupts mast cells, releasing both histamine and serotonin. Waalkes, Coburn, and Terry $^{318}$ have elaborated on their earlier observations and have shown that reserpine liberates serotonin readily from the whole blood, lung, intestine, and brain of both guinea pigs and rabbits. Reserpine, however, liberates histamine readily only from the lung of guinea pigs and the blood, lung, and intestine of the rabbit. There is no decrease of histamine in the guinea pig brain, while the decrease in rabbit brain histamine was not considered significant.

Others have been able to confirm the observation that reserpine lowers histamine levels in rabbit blood but not in other rabbit tissues and have suggested that serotonin released by reserpine may be responsible for the histamine release from rabbit platelets. Haverback and coauthors ${ }^{130,131}$ have shown that serotonin and histamine have differential effects on gastric secretion. Serotonin decreases gastric secretion in the Heidenhane pouch. 5-Hydroxytryptophan decreases spontaneous gastric secretion but does not block the gastric responses to reserpine or histamine. It is still unclear whether serotonin release of histamine would account for these phenomena. Apparently, the effect of reserpine on gastric secretion is peripherally mediated inasmuch as intraventricular injection of reserpine does not increase gastric acidity while peripheral injections do.

Sulser and Brodie ${ }^{293}$ have posed the question, "Is reserpine tranquilization linked to a change in brain serotonin or norepinephrine?" They have concluded that it is related to the former. Their arguments follow:

Although Karki and Paasonen concluded that raunescine caused sedation in rats and lowered brain norepinephrine but not serotonin, the $\mathrm{Na}$ tional Institutes of Health group has evidence that both amines are lowered by this alkaloid.

Although Pletscher and associates offered evidence that the sedative action of 2-benzoquinolizine derivatives in mice was associated with loss of brain norepinephrine, the National Institutes of Health workers found that 20 minutes after injection, brain serotonin levels were also depressed. They argued that serotonin is synthesized rapidly, and this accounts for the higher serotonin levels found 1 hour later.

The findings of Sheppard and Zimmerman of an elevated norepinephrine level in guinea pig brain following reserpine was discounted on the basis of lack of evidence that their fluorometric methods were specific. The National Institutes of Health group concluded that at no time did norepinephrine levels in brain or heart increase after reserpine administration. Brain levels of both norepinephrine and serotonin declined at the same rate and to the same extent.

The evidence for a dissociation between norepinephrine and serotonin is provided by the data of Garattini and Valzelli that reserpine administration to rats exposed to cold caused no sedation and no decline in brain serotonin. The National Institutes of Health workers have shown that under these circumstances, norepinephrine levels decrease, and in confirmation of the Italian workers, they showed no change in brain serotonin or gross behavior. Hypophysectomized rats, however, did show sedation and a fall in brain levels of both amines. Before this question can adequately be settled, more precise psychologic measures of sedation or tranquilization need to be used in connection with the chemical data. It is very difficult to determine in rats exposed to cold whether the animals are sedated or not.

SU-5171 (dimethylaminobenzoyl methylreserpate) releases little brain serotonin despite a marked fall in brain norepinephrine in rabbits. These animals show no evidence of sedation.

Norepinephrine loss centrally is not accompanied by decreased peripheral sympathetic activity. Furthermore, various nonsedative drugs may alter central norepinephrine levels.

Brodie and associates ${ }^{45}, 46,48$ suggest that although total amine levels are lowered, free amine levels are not necessarily, and that the effects on the central nervous system depend upon the levels of free amines at receptor sites. Inasmuch as they claim there is a rapid synthesis of serotonin as compared to norepinephrine, the effects of free serotonin may predominate. Admit- 
tedly, brain norepinephrine levels may fall in a variety of drug-induced states such as morphine mania in cats as shown by Vogt. Inasmuch as reserpine depletes the brain of dopamine and as dopamine is a precursor for norepinephrine and epinephrine, the synthesis of these latter substances must be interfered with. It appears that probably both serotonin and catecholamine depletion may be related to the action of reserpine. The possibility that other mechanisms may be more important should also be kept in mind. Transport mechanisms in addition to binding sites may be involved. Hughes and Brodie ${ }^{152}$ have shown that the entry of serotonin into guinea pig platelets in vivo is retarded by reserpine. Evidence was obtained that this effect was produced via an inhibition of an active transport system. A similar depressant effect of reserpine was noted on the uptake of norepinephrine and epinephrine by platelets. An interesting effect of reserpine has been described by von Euler and Lishajko ${ }^{94}$ in inhibiting the spontaneous release of norepinephrine from isolated nerve granules in vitro. The rate of release was greatly enhanced by DOPA. This observation indicates that the releasing action of reserpine requires an intact nerve cell and that the spontaneous release from isolated nerve granules is actually inhibited.

There is good evidence that in man, reserpine may deplete blood platelets of serotonin. Green, Paasonen, and Giarman ${ }^{118}$ found that blood serotonin values were depressed for 3 weeks after a 3 week course of 1.5 to $3 \mathrm{mg}$. of reserpine given daily to chronic schizophrenics. Hardisty, Ingram, and Stacey ${ }^{126}$ and Haverback and associates $^{129}$ have also noted that reserpine depletes human platelets of serotonin in vivo for as long as 3 weeks following $1 \mathrm{mg}$. of reserpine given intramuscularly. More recently, Freedman and Benton ${ }^{102}$ studied the persisting effects of reserpine in man on blood serotonin levels and pupil size in a group of chronic schizophrenic women. After an oral dose of $10 \mathrm{mg}$. of reserpine, most of the patients became quite ill.
There was vomiting, diarrhea, insomnia, weakness, lassitude, drowsiness, depression, and psychosomatic irritability. These effects began about 2 hours after the drug was given and lasted about 24 hours. The blood serotonin levels fell and continued to be low after two $10 \mathrm{mg}$. oral doses of reserpine for up to 5 weeks. Miosis was present for 7 days after reserpine therapy. These authors concluded that man is exceedingly sensitive to these effects of reserpine. They pointed out that the serotonin-depleting effect on platelets cannot be due. only to the platelets present at the time of drug administration since the life of human platelets is only 8 to 14 days. They suggest a reserpine action on megakaryocytes or their precursors and a sensitizing action on amines in that the first dose of reserpine sensitizes to depletion by a second dose.

It is well known that the 5-hydroxyindole acetic acid content of the urine reflects over-all body serotonin levels. Inasmuch as larger amounts of serotonin are present in intestine and blood than in brain, the former tissues contribute most to urinary levels of 5-hydroxyindole acetic acid. Since this is the major end product of serotonin metabolism, nevertheless, it should be affected by reserpine administration. Valcourt ${ }^{314}$ was able to show that 2 mg. of reserpine given twice daily to psychiatric patients caused an increase in urinary 5-hydroxyindole acetic acid levels. After the first day of treatment, the urinary levels were reduced to control or less.

Brïne and Hinwich ${ }^{50}$, 51 determined the urinary excretion of 5-hydroxyindole acetic acid in psychiatric patients given $2 \mathrm{mg}$. of reserpine twice daily for 14 days, followed by $10 \mathrm{mg}$. of isocarboxazid three times daily in addition and vice versa. An aggravation of psychotic symptoms was reflected by an increase in 5-hydroxyindole acetic acid in the urine. The levels fell slightly during isocarboxazid therapy but rose during the combined reserpine-isocarboxazid period. The total indole-3-acetic acid tended to parallel the alterations in 5hydroxyindole acetic acid. 
Evidence is accumulating that reserpine may not be acting only through its effects on brain amines. Gal, Drewes, and Barraclough $^{106}$ maintained rats on a tryptophandeficient diet. The diet-deficient animals had 70 per cent lower brain levels of serotonin than normal controls. Norepinephrine brain levels were normal. In tryptophandeficient rats, reserpine caused a markedly prolonged depletion of serotonin while norepinephrine levels returned to normal much sooner. Even though there was little serotonin in the brain, a second injection of reserpine produced sedation. These investigators concluded that in the rat, reserpine effects do not require the presence of serotonin in the brain.

Although catecholamine and serotonin levels are depleted from various tissues including brain, the acetylcholine levels of the brain usually rise. ${ }^{215}$ This occurs in most structures, except in the hippocampus. The latter is a site where reserpine may cause stimulation. It is unclear to what extent these changes are related to the sedative effects of reserpine. Malhotra and Das $^{214}$ studied further the effects of reserpine on the acetylcholine content of the heart, ileum, and hypothalamus of the dog. Reserpine increased the acetylcholine levels in all tissues studied. The increase in peripheral tissues was greater than in the hypothalamus. They concluded that the bradycardia and purgative effects of reserpine may be related to peripheral increases in acetylcholine in the tissues involved. The observations that reserpine prolongs diphosphopyridine nucleotide levels further complicates the picture. Burton and colleagues ${ }^{55}$ have observed that reserpine and deserpidine (as well as chlorpromazine and promazine but not meprobamate, pentobarbital, or ethanol) prolong the high diphosphopyridine nucleotide levels in rat liver induced by nicotinamide. Randall, Atkinson, and Iliev ${ }^{261}$ have shown that iproniazid treatment did not modify the reserpine prolongation of diphosphopyridine nucleotide levels of nicotinamide in contrast to its effects in blocking reserpine depletion of serotonin, catecholamines, and overt depression. Much evidence has accumulated that reserpine and chlorpromazine act differently with regard to catecholamine and serotonin levels in the brain. Although both reserpine and the phenothiazines prolong hexobarbital sleeping time in mice, they apparently act differently in this regard as well. Monosodium glutamate further prolongs reserpine enhancement of hexobarbital sleeping time but not that of proclorperazine. ${ }^{181}$

Further evidence of the complexity of reserpine pharmacology is its effect on brain $\gamma$-aminobutyric acid levels. Balzer, Holtz, and Palm ${ }^{13}$ have shown that reserpine causes a long-lasting depletion of $\gamma$-aminobutyric acid in the brains of mice, which correlates with the decrease in electroshock seizure threshold produced by reserpine. Iproniazid pretreatment prevents the depletion of $\gamma$-aminobutyric acid and the decrease in electroshock threshold. Similar results have veen obtained by Palm, Balzer, and Holtz. ${ }^{240}, 241$ The authors felt the decrease in brain $\gamma$-aminobutyric acid was not the actual cause of the lowering effect on electroshock threshold but only an indicator of the interference of reserpine with glutamic acid- $\gamma$-aminobutyric acid metabolism in the brain. To further complicate the picture, MacLean ${ }^{210}$ has observed that after reserpine, there is a decrease in the uptake of radioactive methionine in the hippocampus.

Not all of the actions of reserpine on the brain are specific. Some appear to be related to simple central nervous system depression. For example, Mathe and Kassay ${ }^{219}$ observed that reserpine raised the brain glycogen levels of rats on both acute and chronic administration. Lactic and pyruvic acid levels were also elevated. Other central nervous system depressants such as chlorpromazine and meprobamate had somewhat similar effects, while electroshock or pipradol caused an immediate decrease. These effects, therefore, appear to be related to utilization of substrate by the brain. 
Metabolic fate. Bein ${ }^{23}$ and Maynert ${ }^{20}$ have reviewed the literature on the metabolic fate of reserpine. Both agree that there is little correlation between the levels of reserpine and its known metabolites in the brain and the duration of its pharmacologic effects. The notion that reserpine is "a hit and run drug" therefore may be valid in that the compound causes a central action (such as depletion of brain amines) which persists beyond the presence of the drug in the body. Nevertheless, it cannot be accepted that this is the ultimate mechanism of action of reserpine until the distribution and fate of all reserpine metabolites are known. Theoretically, the most likely possibilities for metabolic breakdown would be hydrolysis of the ester linkage and demethylation. There is very good evidence both in vitro and in vivo that hydrolysis takes place with methyl reserpate and trimethoxybenzoate as the breakdown products. Species differences as well as the route of administration determine the percentage change caused by hydrolysis. For example, on oral administration of reserpine to rats, 22 per cent appears in the urine and 45 per cent in the feces as methyl reserpate. The same dose of reserpine given parenterally results in a urinary level of methyl reserpate of less than 1 per cent of the dose. In other species such as the dog and monkey, regardless of the route of administration, only small amounts of methyl reserpate are found in the excreta and tissues. In addition to methyl reserpate, reserpic acid, syringic acid, and syringoyl methyl reserpate have been recovered after reserpine administration. The other product of simple hydrolysis, 3,4,5trimethoxybenzoic acid, has also been recovered from urine. Maynert does not feel that simple hydrolysis is the primary mechanism of reserpine detoxification. O-Demethylation of reserpine has also been demonstrated. Animals injected with the $\mathrm{C}^{14}$-labeled drug in the 4-methyl group of the trimethoxybenzoyl portion of the molecule exhale 24 per cent of the radioactivity in the breath in a 6 hour period. O-De- methylation derivatives of reserpine (syringoyl methyl reserpate and syringic acid) have also been detected in rat tissues. After intravenous injection, reserpine appears to be rapidly removed from the blood stream. For example, 2 minutes after intravenous injection, only 0.3 per cent remains in the blood. The rest is concentrated in various tissues, especially those with high lipid content. Inasmuch as blood flow to neutral fat is rather low, these sites reach peak concentrations in about 4 to 6 hours. Of considerable interest, reserpine-induced sedation does not coincide with its peak concentration in the brain as a whole or in its separate parts. Sheppard and associates ${ }^{284}$ also used randomly tritum-labeled reserpine in order to study its distribution. The results obtained were similar to those previously reported using $\mathrm{C}^{14}$-labeled reserpine in the trimethoxybenzoic acid portion of the molecule. ${ }^{283}$ The conclusion that the intensity of central pharmacologic effects of reserpine bears no relation to its concentration in brain was further supported by this study. The authors also pointed out that the lowering of serotonin and catecholamine levels in the brain, as reported by others, also is poorly correlated with the gross observable effects of reserpine. One of the major criticisms of most chemical studies is that the chemical events in the brain are correlated with gross observation of the animals rather than specific psychologic behavioral tests. It is obvious that peripheral symptoms may influence an investigator in assuming the animal is sedated.

Reserpine apparently can cross the placental barrier. Infants of mothers who received the agent prior to delivery may show severe toxic symptoms and nasal discharge and occasionally may die from anoxic complications. ${ }^{52,266}$

Plummer, Sheppard, and Schulert ${ }^{256}$ have summarized their findings on the metabolism of reserpine. Their conclusions are in agreement with what has been reported previously, in that the metabolites of reserpine include trimethoxybenzoate, methyl 
reserpate, reserpic acid, syringomethyl reserpate, syringic acid, and carbon dioxide. There appears to be no relation between the sensitivity of any given species and the pattern of metabolism of reserpine. The rate of uptake of reserpine by the brain resembles that of other tissues except neutral fat. There does not appear to be a differential distribution of reserpine in different brain areas. It is of interest that Plummer, Sheppard, and Schulert were able by very sensitive radioactive labeling procedures to show the presence of reserpine in the brain for at least 48 hours after injection of small pharmacologically active doses. This latter observation is of crucial importance and raises some doubts on the concept of a hit and run action of reserpine. Again, more precise behavioral correlations with chemical data will provide evidence necessary to accept or reject this concept.

Adverse effects. Hollister ${ }^{141}$ has reviewed the adverse effects of the Rauwolfia alkaloids. Several categories have been described, ${ }^{177}$ which comprise behavioral effects including depression and suicide, extrapyramidal symptoms, autonomic system effects, and allergic and endocrine effects. Depression is both the most common and the most serious behavioral effect which may lead to suicide. Occasionally, adverse psychotic symptoms may be evoked. The extrapyramidal symptoms usually produced by reserpine are those of a classic type of parkinsonism. In contrast to the phenothiazines, the dystonias are very rare. Rare choreoathetotic and cerebellar syndromes have been seen. Reserpine has been reported to lower the convulsive threshold in man ${ }^{176,235}$ as well as to control seizures. ${ }^{190}$

Autonomic system effects include increased parasympathetic and decreased sympathetic effects. These consist of bradycardia, flushing, salivation, diarrhea, nasal congestion, hypotension and syncope, and activation of a peptic ulcer or acute gastric erosions. Interestingly, the Rauwolfia alkaloids do not produce many allergic phenomena. Both thrombocytopenic and non- thrombocytopenic purpura have been observed rarely. Allergic disorders may be aggravated by these agents. Patients taking the Rauwolfia drugs may gain weight, part of which is fluid; frank edema may occur. These agents may act to release antidiuretic hormone.

The endocrinologic actions of reserpine may be marked (see section on endocrines). In men, feminizing effects may occur, with loss of libido and impotence. Reserpine may antagonize the effects of thyroxine. In fact, it has been used therapeutically in selected patients with thyrotoxicosis.

\section{Meprobamate}

Meprobamate is a 2-methyl-2-n-propyl-1, 3-propanediol dicarbamate. It was synthesized by Ludwig and Piech in 1950. Its structure is as follows:<smiles>CCCC(C)(COC(N)=O)C(N)=O</smiles>

It is a white, bitter powder with a mild characteristic odor. It is freely soluble in alcohol and various organic solvents but only slightly soluble in water.

The principal pharmacologic effect of meprobamate is depression of the central nervous system. Berger ${ }^{2 \tau}$ described meprobamate in animals as a mephenesin-like drug producing skeletal muscle relaxation, protection against pentylenetetrazol, strychnine, and the tonic phase of electroshock seizures, prolongation of barbiturate anesthesia, and a sedative effect which causes monkeys to be handled more easily. Since then, much has been written describing the effects of this agent in animals and man. To say the least, meprobamate is a controversial drug. Its widespread clinical acceptance is probably due to a very complex set of circumstances, including a desire on the part of many physicians for a nonbarbiturate sedative, efficient drug advertising, and the apparent effectiveness of meprobamate in relieving anxiety without producing too much drowsiness. 
Antianxiety effects. Laties and Weiss ${ }^{197}$ have reviewed the available literature on the effectiveness of meprobamate in the treatment of anxiety. The large percentage of published uncontrolled studies with this agent led them to the conclusion that no good evidence exists on the unique superiority of meprobamate as an antianxiety agent. The reader is referred to their critical survey of the literature to 1958 and their analysis of the various errors in clinical design of experiments. Even though many investigators thought they were conducting controlled experiments, in reality they were not. Their review is a striking testimonial to the insufficiency of all of us as members of the medical profession in knowledge of proper clinical research design.

Even if one accepts the statement that meprobamate is an effective antianxiety agent, it still is pertinent to ask if it is pharmacologically unique in this regard. Laties and Weiss concluded that there was no good evidence available to suggest it was better than a barbiturate. In fact, there was some evidence that it might not be as good. Since then, several other studies have been published which bear on this question. The study of Moyer and associates ${ }^{232}$ in which meprobamate ( 800 to $2,400 \mathrm{mg}$. per day), phenaglycodol ( 600 to $1,600 \mathrm{mg}$. per day), and benactyzine and its related derivative ( 3 to $8 \mathrm{mg}$.) were compared to phenobarbital (120 mg. per day) and a placebo does not indicate any unique over-all effectiveness of meprobamate in relieving anxiety and tension in psychoneurotics. Even when meprobamate was compared to these relatively low doses of phenobarbital, it was noted that patients who responded to phenobarbital also responded to meprobamate. There was no correlation between severity of the symptoms and responsiveness to both agents. Similarly, there was no significant difference in the degree of response in patients reacting favorably to both drugs. Meprobamate was the most effective of the four new experimental drugs tested (excluding phenobarbital), but subjective improvement was better than objective improvement. Objectively, the response to all four agents was comparable. The authors pointed out that although meprobamate was effective in approximately 25 per cent more patients than phenobarbital, the dosage of the latter drug was rather low. They suggested that larger doses probably would have produced an increase in response comparable to the four other experimental drugs. Another comparative drug study is that of Rickels and co-workers. ${ }^{265}$ These investigators determined the effects of amobarbital sodium (30 mg.), meprobamate (400 mg.), proclorperazine ( $5 \mathrm{mg}$.), and a placebo given four times per day for 2 weeks in a random order to psychoneurotic outpatients with predominant symptoms of anxiety, tension, and mild depression in the absence of evident organic disease. A series of psychologic and psychiatric improvement scales was used to evaluate the subjects. It was concluded that all four treatments significantly influenced the patients during the first 2 weeks. Thereafter, only the three active drugs significantly gave better results. Therapy with meprobamate produced the most marked change toward improvement. It is regrettable that a larger dose of amobarbital was not used. Dickel and colleagues ${ }^{77}$ compared the effects of meprobamate ( $400 \mathrm{mg}$.), chlordiazepoxide (10 mg.), and phenobarbital (50 mg.) in a double blind, crossover fashion in 36 working outpatients with presenting symptoms of anxiety. The drugs were given for 2 weeks each on a schedule of three or four times daily. All of the patients were evaluated on the basis of psychiatric interviews and about half on the degree of relaxation in response to faradic stimulation, ability to perform tasks, solve problems, etc. Meprobamate was found to be most effective in reducing anxiety in the working patient, while chlordiazepoxide appeared more useful in more severe emotional disorders. In this study, phenobarbital, while useful in some cases, had the least over-all effectiveness. 
Soporific effects. Meprobamate appears to be effective in promoting sleep. Lasagna $^{193}$ compared a series of sleep-inducing agents including a placebo (lactose), methyprylon ( 0.25 and $0.5 \mathrm{Gm}$.), meprobamate $(0.4$ and $0.8 \mathrm{Gm}$.), pentobarbital ( 0.1 and $0.2 \mathrm{Gm}$.), secobarbital (0.1 and $0.2 \mathrm{Gm}$.) and phenobarbital (0.1 and 0.2 $\mathrm{Gm}$.) on the promotion of sleep in patients with various chronic diseases. He concluded that meprobamate has hypnotic properties resembling phenobarbital. The most satisfactory results in promoting sleep were obtained with $0.2 \mathrm{Gm}$. of secobarbital and pentobarbital. Batterman and coauthors $^{21}$ compared phenobarbital (15 mg.), butabarbital ( $15 \mathrm{mg}$.), acetylcarbromal (200 mg.), glutethimide (250 Gm.), meprobamate ( $400 \mathrm{mg}$ ), and proclorperazine ( $5 \mathrm{mg}$.) given three to four times daily to nervous outpatients for daytime sedation and nighttime hypnotic effects. Of these, butabarbital was the most satisfactory, while meprobamate was least satisfactory, especially as a daytime sedative. The authors pointed out that effectiveness for daytime sedation does not necessarily parallel effectiveness for nighttime hypnosis. For a single agent with both effects, butabarbital appeared to be the best. Rickels and associates, ${ }^{265}$ in comparing meprobamate ( $400 \mathrm{mg}$.) to amobarbital (30 mg.), proclorperazine ( $5 \mathrm{mg}$.), and a placebo given four times daily for 2 weeks to psychoneurotics, noted that although amobarbital produced more daytime drowsiness, meprobamate was most effective in relieving insomnia. The authors felt the beneficial effect could not be due to a primary sedative (hypnotic) effect but might be due to skeletal muscle relaxation.

Skeletal muscle relaxant effects. There appears to be some evidence that meprobamate relaxes skeletal muscle tension in animals and man. ${ }^{301}$ The critical question is whether this effect is due to a specific skeletal muscle relaxant action or to sedation. Surprisingly, very few critical studies have been made in which several central nervous system depressants were compared to meprobamate. Vuzuka ${ }^{315}$ studied the muscle relaxant effects of methocarbamol (1,250 mg.), meprobamate (400 mg.), and zoxazolamine $(500 \mathrm{mg}$.) given orally three or four times daily to a series of patients with neuromuscular disorders. He used clinical observations, photographic recordings, and electromyographic and myometric studies to evaluate the degree of skeletal muscle relaxation. The patients showed varied degrees of muscle spasticity, ankle clonus, and spasms. Meprobamate appeared to be the most effective, methocarbamol second, and zoxazolamine least active in relieving spasticity. Nyquist, Comarr, and Bors ${ }^{237}$ compared meprobamate (800 mg.), phenobarbital (50 mg.), zoxazolamine ( $500 \mathrm{mg}$.), and a placebo given orally three or four times daily to patients having spasticity associated with spinal cord injuries. After 7 days of treatment, all three agents were more effective than the placebo in having a relaxant effect on skeletal muscle. In these doses, meprobamate was more effective than phenobarbital, while zoxazolamine was least active. These investigators concluded that phenobarbital was a valuable substitute for meprobamate. Lamphier and Pine ${ }^{191}$ compared meprobamate ( 400 to $800 \mathrm{mg}$.), emylcamate ( 200 to $400 \mathrm{mg}$.), and a placebo given usually three times daily for 2 to 4 days to hospitalized patients and 1 to 60 days to office patients with spastic muscular disorders and psychosomatic symptoms. Both drugs were more effective than a placebo, but emylcamate was superior to meprobamate.

A particularly important unpublished study ${ }^{*}$ by Bayliss deserves special attention even though meprobamate itself was not studied. This investigator studied the effects of mephenesin, methocarbamol, zoxazolamine, chlorzoxazone, chlormethazone, and secobarbital given acutely in adequate therapeutic doses on the electromyograms of paraplegic patients with muscle spasms and hypertonicity. He concluded that there

*S. M. Bayliss: Personal communication, 1961. 
was no marked difference in effect of the skeletal muscle relaxants compared to secobarbital.

Perlstein, Turner, and Elam ${ }^{247}$ compared the effects of chlorpromazine ( 25 to $50 \mathrm{mg}$. intravenously or intramuscularly), meprobamate ( $400 \mathrm{mg}$. intramuscularly), pentobarbital ( $200 \mathrm{mg}$. intramuscularly), and an experimental drug, MR 710 (900 mg. orally), in 7 patients with tetanus using clinical and electromyographic criteria. Chlorpromazine and pentobarbital reduced but did not eliminate the spontaneous electromyographic activity and the spasms triggered by various stimuli. Both drugs, however, caused sleepiness to the point of coma. Meprobamate eliminated the spontaneous activity at rest and dramatically stopped the spasms produced by external stimuli. It did not prevent spasms induced by internal stimuli. MR 710 diminished the spontaneous activity at rest as well as the induced spasms. It was more soporific than meprobamate. Meprobamate had the least soporific effect. In a more recent study, Perlstein, Stein, and Elam ${ }^{246}$ continue to favor the use of meprobamate intramuscularly over chlorpromazine and the barbiturates in the treatment of tetanus. Difficult cases usually require multiple medications and other supportive therapy.

As might be anticipated from animal studies, meprobamate has been found to control certain patients with petit mal and related epilepsies. Generally, meprobamate is ineffective in grand mal and, like other drugs used in petit mal, may sometimes aggravate grand mal attacks. Perlstein ${ }^{245}$ considers meprobamate less effective than trimethadione but as effective as paramethadione. Meprobamate has an advantage over these agents in that it has fewer serious effects.

Effects on various objective behavioral tests. Marquis and colleagues ${ }^{217}$ studied the effects of single doses of a lactose placebo (1,400 mg.), meprobamate (800 mg.), Damphetamine (15 mg.), meprobamate (800 mg.) plus alcohol ( 2 ounces of 86 proof whiskey), and a lactose placebo
(1,400 mg.) plus alcohol ( 2 ounces of 86 proof whiskey) in a series of normal subjects given various objective behavioral tests including reaction time, driving skill, steadiness, and visual performance using an Ortho-rater. Meprobamate had no significant depressant effect, while alcohol did impair performance on some of the tests. Combinations of meprobamate and alcohol did not show any enhanced effect. Subsequent studies on the chronic administration of $1,600 \mathrm{mg}$. of meprobamate per day for 3 to 4 weeks also showed that meprobamate alone had no adverse depressant effects. ${ }^{166,167}$ However, single doses of 800 and 1,600 mg. of meprobamate were found by Kornetsky ${ }^{183}$ to produce a significant impairment of learning and reaction time in normal subjects tested on a multiple stimulus-response apparatus. Three procedures were used: simple motor behavior, choice reaction time, and learning. This investigator also studied the effects of phenobarbital (60 and $120 \mathrm{mg}$.), D-amphetamine ( 5 and $15 \mathrm{mg}$.), and a placebo. In contrast to the significantly impaired performance after meprobamate, the latter agents did not significantly alter motor coordination, reaction time, or learning. The failure of $\mathrm{D}$-amphetamine to facilitate performance of normal nonfatigued subjects is in general agreement with other studies in the literature.

Melander $^{222}$ also studied the effects of meprobamate (400 mg.), placebo, and amobarbital (100 mg.) given in single doses on simulated automobile driving. There was no significant difference between meprobamate and the placebo, while amobarbital did depress driving skill. It should be noted that the dosage of meprobamate was rather small compared to that of amobarbital. Loomis and West ${ }^{206}$ also studied the effects of a series of drugs on simulated driving skill. The agents used were corn starch placebo (200 mg.), secobarbital (100 mg.), chlorpromazine (50 mg.), meprobamate (400 mg.), and phenaglycodol (300 mg.) given in several doses under controlled conditions including a light 
breakfast and a standard lunch. All drugs except the placebo and phenaglycodol produced a significant impairment of performance on the test apparatus. Secobarbital produced the most intense impairment. Chlorpromazine produced an impairment of function after a delay of several hours. Meprobamate also produced delayed impairment after the first dose and prompt impairment after the second dose.

Zirkle and colleagues ${ }^{337}$ have shown that $400 \mathrm{mg}$. of meprobamate four times daily for 1 week in normal subjects had an increased depressant effect on coordination and judgment when combined with 95 per cent ethyl alcohol in sufficient quantity to produce an estimated 0.05 per cent blood alcohol level. These investigators found that meprobamate alone, alcohol alone, and meprobamate plus alcohol, in that order, increasingly impaired performance significantly from that of a placebo. There was no significant difference in the blood alcohol levels of alcohol treatment alone or with meprobamate, indicating that meprobamate did not enhance the absorption or interfere with the metabolism of alcohol. The results indicated a definite additive and possibly a potentiating effect of meprobamate on alcohol. If the placebo percentage of success was counted as 100 , the comparative percentages of success in the drug states were: meprobamate, 95; alcohol, 90; and meprobamate-alcohol, 80. The expected deficit for the combination of drugs would have been 85 per cent. Inasmuch as 80 per cent successful performance was obtained, a true mild potentiating effect of meprobamate may have been observed. A study by Kopmann and Hughes ${ }^{182}$ using rats would support the above human data. The latter investigators studied the effects of various central nervous system depressants alone and in combination with ethyl alcohol on anxiety, discrimination, and extinction of a learned response involving two choice discriminations in a three chamber shock avoidance box. Meprobamate, phenaglycodol, and alcohol decreased anxiety, discrimination, and the ability to respond. As expected, chlorpromazine in doses which were ineffective alone markedly potentiated the depressant effects of alcohol. Unexpectedly, the authors found that meprobamate as well as phenaglycodol and pentobarbital potentiated the depression of behavior induced by ethyl alcohol. The depressant effects of reserpine were merely additive to those of ethyl alcohol. These findings merit further study. Tentatively, the evidence suggests that meprobamate and alcohol may not merely show additive depressant properties but true potentiation. It is of interest that Joyce and colleagues ${ }^{160}$ have obtained evidence that a similar potentiation rather than simple addition is present in normal subjects who ingested phenobarbital and ethyl alcohol.

The effects of various central nervous system depressants on the disruptive effects of anxiety on performance of a perceptual motor task (keeping a pointer on a target) have been studied by Holliday and Dille. ${ }^{139}$ Anxiety was produced by random "punishment" for being off target. The punishment consisted of a slight electric shock, a blast of air on the neck, or a horn blast. The drugs tested included meprobamate ( 800 mg.), pentobarbital (100 mg.), chlorpromazine ( $50 \mathrm{mg}$.), and a placebo given to patients on a double blind basis. Of the drugs tested, only meprobamate tended to abolish the disruptive effects of anxiety on learning as measured in this study.

In order to help answer the question of whether pilots of airplanes should be allowed to fly while under the influence of various tranquilizers, McGuire and Leary ${ }^{209}$ studied the effects of a placebo, promazine (50 mg.), and meprobamate (400 mg.) given four times daily on stress tolerance. Various tests of circulatory "overshoot" or compensation to the Valsalva maneuver, continuous positive pressure breathing, the tilt table, and exercise tolerance were used. As might be expected of adrenergic blocking agents, promazine caused a reduction of stress tolerance. However, meprobamate also depressed stress tolerance. As the degree of stress increased, the limitation of 
response became severe with both groups of tranquilizing agents. Large doses of both active pharmacologic agents caused even more severe limitations of circulatory compensation.

An investigation by Lienert and Traxel ${ }^{203}$ showed the galvanic skin response to emotion-laden statements to subjects given meprobamate or alcohol was significantly reduced. These drug effects were more marked with highly emotional subjects.

Uhr and Miller ${ }^{312}$ studied the effects of single doses of emylcamate ( 400 to 800 mg.), meprobamate ( $800 \mathrm{mg}$.), and matching placebos on various psychologic tests examining stress reactions in normal male volunteers. No drug effects were found on performance under either physical stress or threat of stress situations. Meprobamate had a consistent effect in raising galvanic skin resistance, which is consistent with the findings of Laties ${ }^{196}$ that meprobamate decreases palmar sweating, but it is not consistent with a previous study of Marquis and colleagues ${ }^{217}$ that determined meprobamate increases sweating. Both meprobamate and emylcamate led to feelings of less activity, the latter being more potent.

Jonsson and Andersén ${ }^{158}$ studied the effects of emylcamate, meprobamate, and a placebo given on a double blind basis to psychiatric patients ready for discharge from a hospital. A battery of psychologic tests which included sixteen variables was used. Meprobamate in single doses of 1,200 and $1,800 \mathrm{mg}$. significantly impaired performance on the vigilance test, various visual-motor dexterity tests, and grip strength. Single doses of 1,200 mg. of emylcamate caused no impairment of performance, while $1,800 \mathrm{mg}$. had a depressant effect similar to meprobamate. Both drugs, even in doses of $1,800 \mathrm{mg}$., had no effect on the flicker fusion test.

Actions on the human electroencephalogram. In man, oral doses of 1,600 and $2,000 \mathrm{mg}$. of meprobamate produce barbiturate-like fast waves of 20 to 30 cycles per second in the cortical electroencephalogram, especially in the parietal areas. ${ }^{248}$
Such electroencephalographic fast wave activity also has been observed by others. ${ }^{279}$

Bokonjic and Trojaborg ${ }^{35}$ have studied the effect of a wide range of doses of meprobamate ( 15 to $400 \mathrm{mg}$. per kilogram, orally) on the electroencephalogram of patients during treatment, during intoxication, and after abrupt withdrawal. In certain respects, meprobamate and the barbiturates have similar effects on the electroencephalogram. Both drugs induce fast wave activity which is more pronounced during drowsiness and disappears during sleep. Both drugs show evidence of tolerance to these electroencephalographic changes. After abrupt withdrawal of both agents, paroxysmal electroencephalographic changes are noted during photic stimulation. In confirmation of the work of Pfeiffer and colleagues, the cortical distribution of the fast frequency activity seems to differ. After meprobamate, the electroencephalographic fast wave activity tends to be most pronounced in the parietal areas, while after barbiturates, it is most pronounced frontally, frontotemporally, or parietally. After withdrawal of meprobamate, the paroxysmal electroencephalographic changes rarely persist as long as 2 weeks. After withdrawal of barbiturates, the changes may last 2 months. The principal differences between the electroencephalographic effects of meprobamate and barbiturates occur after ingestion of toxic doses. After meprobamate poisoning, the fast waves persist even during the period of unconsciousness. Frequencies of less than 6 cycles per second rarely are seen. The electroencephalogram usually is normal 48 hours after doses as large as $400 \mathrm{mg}$. per kilogram orally. The electroencephalographic fast wave activity persists only with small doses of barbiturates. After large toxic doses, the fast wave activity is replaced by 2 to 6 cycles per second activity of high amplitude. It may take weeks for the electroencephalogram to become normal, although admittedly this could be secondary to brain damage caused by hypoxia resulting from severe respiratory depression. 
Metabolic fate. The metabolic fate of meprobamate was investigated initially in animals and man by Berger ${ }^{27}$ and Agranoff, Bradley, and Axelrod. ${ }^{3}$ Subsequently, Walkenstein and colleagues ${ }^{320}$ extended these studies. When meprobamate is administered orally to dogs, it is readily absorbed into the blood stream. Peak blood concentrations are observed 1 to 2 hours after administration. The levels then decline for 10 or more hours. Significant amounts appear in the urine within 30 minutes after an oral dose, and most of the meprobamate is excreted within 24 hours, especially in rats and dogs. Feces contain less than 10 per cent of the dose. Approximately 50 per cent of the amount present in blood is unchanged meprobamate. After administration to rats, the lungs, kidney, and liver show the greatest drug concentration. The major metabolite, accounting for 60 per cent of the administered dose to dogs, is hydroxymeprobamate, which does not exhibit meprobamate-like activity. About 10 to 12 per cent of the meprobamate in urine is unchanged; the rest are metabolites conjugated with glucuronic acid. Agranoff, Bradley, and Axelrod showed that following single doses of $1,600 \mathrm{mg}$. of meprobamate orally to 4 human subjects, the drug is excreted relatively slowly for at least 48 hours after administration. About 12 to 20 per cent of the administered meprobamate was excreted unchanged in the urine. Walkenstein and colleagues obtained similar findings in 9 human subjects given $1 \mathrm{Gm}$. of meprobamate as a single oral dose. About 0.66 per cent of the meprobamate given was excreted unchanged in the first 2 hours. The excretion rate then doubled during the next 2 hours and remained at this level to the twelfth hour. By this time, about 7 per cent of the drug was excreted unchanged. A gradual decrease was observed in the excretion rate from 24 to 48 hours, which accounted for only 11.5 per cent unchanged meprobamate over the 48 hour period. Recently, Ludwig and associates ${ }^{20 \tau}$ have reported that 2hydroxymethyl-2-propyl-1,3-propanediol di- carbamate is not the major unconjugated metabolite of meprobamate, as reported previously by others. These investigators have established the metabolite to be 2 methyl-2( $\beta$-hydroxypropyl)-1, 3-propanediol dicarbamate. Compared to meprobamate this agent is pharmacologically impotent. Emmerson, Miya, and Yim ${ }^{91}$ studied the distribution of $\mathrm{C}^{14}$-labeled meprobamate in the rat after intraperitoneal administration. The distribution of meprobamate was similar in the cerebrum, cerebellum, midbrain, and hindbrain (a section just at the anterior border of the pons to one below the medulla). In nephrectomized rats, three compounds, a glucuronide, hydroxymeprobamate, and meprobamate, were found in the blood, but only meprobamate was present in the brain. Phillips, Miya, and Yim ${ }^{249}$ administered meprobamate to rats for a period of 35 days. Definite tolerance was shown to develop, as evidenced by a decrease in motor activity within 1 hour after meprobamate administration. Blood and brain levels of $\mathrm{C}^{14}$-labeled meprobamate in the carbamate positions were approximately the same within 1 hour after drug administration. Subsequently, the level of meprobamate in the tolerant animals decreased much more rapidly than in nontolerant animals. A marked increase in urinary excretion of the glucuronide metabolites of meprobamate was observed in the tolerant animals. It would appear that the tolerance to meprobamate indicated by a decrease in motor activity within 1 hour was due to cellular adaptation of the nervous system, while the enhanced metabolism of the drug was responsible for a marked shortening of its duration of action. Other investigators have shown that tolerance to the barbiturates may also involve an increased rate of metabolism of the active compound to pharmacologically inactive metabolites ${ }^{65}$ Cross tolerance of meprobamate with other pharmacologic agents has been demonstrated presumably by increasing the activity of the enzyme systems responsible for the metabolism of meprobamate. ${ }^{64,164,}$ 165 
Physical dependence. It is now well known that meprobamate may produce physical dependence in animals and man if taken in sufficient dosage. Swinyard, Chin, and Fingl ${ }^{300}$ studied the effects of chronic oral administration of meprobamate to albino mice using the threshold to low frequency electroshock as an index of central nervous system excitability. After several days of treatment, marked tolerance developed to the usual seizure threshold-elevating effect of meprobamate. After 12 to 16 days of chronic meprobamate administration, the seizure thresholds were below normal, indicating withdrawal hyperexcitability. Withdrawal hyperexcitability was detectable 4 and 8 hours after drug withdrawal and subsided within 28 hours. This was in marked contrast to withdrawal caused by chronic ethanol or phenobarbital administration. With the latter agents, there is little hyperexcitability in mice for the first 24 hours, but it is maximal on the second or third day and declines progressively over the next 4 days. ${ }^{211}$ In dogs, gross signs of withdrawal from chronic meprobamate administration twice daily occurred 12 to 16 hours after the last dose. Grand mal seizures occurred about 24 hours after withdrawal, with three of four animals dying after several seizures. ${ }^{93}$ A previous study by Fraser and Isbell in dogs chronically administered various barbiturates also showed these agents produced an abstinence syndrome. Secobarbital, amobarbital, pentobarbital, and barbital were given once daily, except for one group given pentobarbital five times a day. Secobarbital and amobarbital produced mild or inconsistent signs of abstinence, probably caused by the fact that compounds are short acting and were given only once a day. The animals given pentobarbital in divided daily doses showed more marked withdrawal symptoms, including grand mal convulsions, while barbital produced the most marked abstinence syndrome, with convulsions within 21 to 48 hours. During the first few weeks of intoxication, all animals showed evidence of tolerance development to the sedative effects. Superficially, it would appear that in dogs, withdrawal to the shorter-acting barbiturates is less severe than in man, ${ }^{156}$ but this is probably related to the lack of maintaining persistent 24 hour blood levels of these agents in the dog.

Bulla, Ewing, and Buffaloe ${ }^{\tilde{3}}$ extended the previous study of Haizlip and Ewing ${ }^{124}$ in patients using smaller doses of meprobamate over a longer period of time. In a controlled study using psychotic patients, it was shown that $2,400 \mathrm{mg}$. or less of meprobamate per day did not lead to withdrawal symptoms in the majority of patients. These findings confirm those of Boyd and colleagues ${ }^{38}$ who failed to show withdrawal symptoms following similar doses of meprobamate given to more elderly patients. On the other hand, that doses of 3.2 and $6.4 \mathrm{Gm}$. of meprobamate and more per day given chronically led to definite physical dependence and withdrawal symptoms was shown by Haizlip and Ewing. ${ }^{124}$ These investigators conducted a double blind study with three identical groups of male psychiatric inpatients who were given placebo or meprobamate at two dose levels (3.2 and 6.4 Gm. per day). Marked sedative effects were observed with meprobamate for the first few days. Of 47 patients, 35 showed a staggering gait or inability to stand or walk without falling. These effects gradually diminished over the next 7 to 10 days, indicating tolerance development. At the end of 40 days of daily treatment, all patients were switched to a placebo. A total of 44 of 47 patients showed objective evidence of withdrawal. The typical syndrome of meprobamate withdrawal consisted of insomnia, vomiting, tremors, muscle twitching, anxiety, anorexia, and ataxia. Eight patients had hallucinations, anxiety, and tremors resembling delirium tremens between 36 and 48 hours after withdrawal of meprobamate. Three of the patients developed grand mal seizures within this same period of time after drug withdrawal. The results obtained were statistically significant. Hollister and Glaz- 
ener $^{144}$ showed that only 10 of 60 patients switched from usual therapeutic doses of meprobamate to placebo had a recrudescence of symptoms. A definite withdrawal syndrome was observed in 16 of 21 patients with mean daily doses of $5.8 \mathrm{Gm}$. A similar picture was observed following abrupt withdrawal of equally large doses of meprobamate combined with promazine ( 600 to $800 \mathrm{mg}$. daily). The plasma levels of high doses of meprobamate daily in 23 patients after withdrawal indicated a halflife of meprobamate of approximately 24 hours.

Untoward effects and toxicity. Considering its widespread use, meprobamate in usual therapeutic doses produces relatively few adverse effects. Serious effects such as thrombocytopenic purpura, leukopenia, and aplastic anemia have been reported, but these are rare. Allergic symptoms have been observed. These usually occur after a few doses of the drug. The incidence of reactions is not apparently related to a previous history of allergy. The mild reactions consist of an urticarial or erythematous maculopapular rash which may be confined to the groin or generalized. Acute nonthrombocytopenic purpura with fever and edema has also been seen. Serious reactions including marked fever, fainting spells, angioneurotic edema, and bronchial spasm may also occur.

Ingestion of large amounts of meprobamate, particularly in cases of overdosage with suicidal intent, may result in coma, cardiovascular shock, and respiratory collapse and death. Cann and Verhulst analyzed the case reports of 93 patients with meprobamate overdosage. In this series, there were no deaths, although deaths have been reported by others. Drowsiness, lethargy, stupor, and coma in adults occurred in doses of 4 to $9.6 \mathrm{Gm}$., with 1 patient ingesting $19.2 \mathrm{Gm}$. Accidental overdosage of meprobamate in children does occur, but not as much as might be expected, in part perhaps as a result of the bitter taste of the drug. The clinical picture of overdosage in children resembles that in man.
It would appear that suicide attempts with meprobamate are not too successful (eight in the literature to 1961) because of the large quantities of the drug necessary to produce coma and death. Deaths have followed ingestion of as little as 12 and 20 $\mathrm{Gm}$., but doses up to $40 \mathrm{Gm}$. have been taken by some patients with recovery. ${ }^{141}$ Kamin and Shaskan ${ }^{163}$ reported a patient who ingested $47.6 \mathrm{Gm}$. and died of aspiration and pulmonary congestion.

\section{Chlordiazepoxide}

Chlordiazepoxide is a member of a new class of chemicals of unusual structure. The compound is 7-chloro-2-methylamino-5phenyl-3H-1,4-benzodiazepine-4-oxide hydrochloride, as shown below:

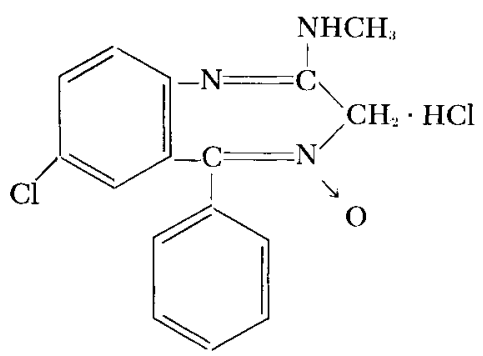

It was synthesized by Sternbach and Reeder. The colorless crystalline material is highly soluble in water. It is unstable in solution, and the powder must be protected from light.

Randall and colleagues ${ }^{262}$ described this agent as having sedative, anticonvulsant, and skeletal muscle relaxant properties but weak hypnotic effects in animals. It produces taming of vicious animals and an appetite-stimulating effect in rats and dogs. These investigators described chlordiazepoxide as qualitatively similar to meprobamate as a tranquilizer but more potent. Liberson $^{202}$ has pointed out that the behavioral effects of chlordiazepoxide in hungry, fixated rats appears to be different from those of other drugs used as tranquilizers in man. For example, chlorpromazine tends to suppress the avoidance of the grid shock. Meprobamate tends to de- 
lay the reactions of the rat and slow the rate of learning. In confirmation of Feldman's results, chlordiazepoxide tends to prevent fixations in rats. In contrast to the other tranquilizers, the drug does not suppress avoidance to grid shock and shortens the reaction times even more than psychic energizers. The role of the appetitestimulating effect of chlordiazepoxide in rats in these behavioral changes was not determined. No data are as yet available as to whether the psychopharmacologic effects of chlordiazepoxide in rats apply to humans.

Sedative effects. In man, chlordiazepoxide has mild sedative effects which parallel the effects observed in animals. ${ }^{302}$ Relatively few clinical reports have appeared in which double blind techniques or accurate comparisons to standard sedatives have been made. Scherbel ${ }^{272}$ studied the action of chlordiazepoxide and a placebo in various diseases. Usually, $10 \mathrm{mg}$. three times daily was given to patients with a variety of organic and functional disorders. He concluded the compound is effective in relieving anxiety and its accompanying somatic reactions. The adverse effects included increased drowsiness and need for sleep, increased appetite, ataxia, muscle incoordination and weakness, decreased libido, decreased tolerance to alcohol, and in some patients, aggravation of a depressive reaction. Elderly patients were found to be very sensitive to even small doses. In addition, a cumulative effect was observed in some patients. This is in accord with Hollister, Motzenbecker, and Degan's ${ }^{145}$ observations that the plasma levels of the drug indicate a half-life of approximately 48 hours following very large daily doses ( 300 to $600 \mathrm{mg}$.). These are about 10 times the usual therapeutic dose.

Anticonvulsant effects. Contrary to the reports of Kaim and Rosenstein and of Rosenstein $^{302}$ that chlordiazepoxide is an anticonvulsant in man, Livingston, Pauli, and Murphy ${ }^{204}$ found it ineffective in doses of 70 to $125 \mathrm{mg}$. per day in resistant epileptics who also failed to respond to standard anticonvulsant drugs. Some improvement in behavior was noted in a few patients with hyperkinetic manifestations. In addition, these latter investigators found the agent produced numerous untoward effects, including anorexia, drowsiness, aggressive destructive behavior, ataxia, and a decrease in potency.

Metabolic fate. Unfortunately, relatively few data are available on the blood levels and metabolites of chlordiazepoxide. It appears in the blood a few minutes after oral administration, and peak levels are attained about 2 hours later. ${ }^{335}$ Both in rats and man, $\mathrm{C}^{14}$-labeled chlordiazepoxide was rapidly absorbed from the intestine. ${ }^{178}$

Part of the drug was secreted into the stomach and reabsorbed, while part was excreted into the feces. These effects persisted for about 48 hours in the rat but longer in man. The agent was extensively metabolized in both species. None of the $\mathrm{C}^{14}$-labeled material appeared as $\mathrm{C}^{14} \mathrm{O}_{2}$ in the lungs. Rats excreted predominantly basic while humans excreted acidic metabolites. Hollister, Motzenbecker, and De$\operatorname{gan}^{145}$ have estimated on the basis of plasma levels following abrupt withdrawal of very large daily doses ( 300 to $600 \mathrm{mg}$.) to psychiatric patients that chlordiazepoxide has a plasma half-life of approximately 48 hours.

Physical dependence. To date, there is no evidence that the chronic ingestion of usual therapeutic doses of chlordiazepoxide leads to physical dependence. It is of interest that the drug can relieve the symptoms of delirium tremens or alcohol withdrawal. It is pertinent, therefore, to ask if the drug can substitute for alcohol or act through some other unknown mechanism as do the substituted phenothiazines. That chlordiazepoxide in enormous dosage is capable of producing physical dependence and withdrawal symptoms is evident from a study by Hollister, Motzenbecker, and Degan. ${ }^{145}$ These investigators studied the effects of large doses (100 to $600 \mathrm{mg}$.) of chlordiazepoxide given daily for 1 to 7 months to 36 hospitalized psychiatric pa- 
tients. Some clinical improvement was noted in 9 patients. Dizziness, weakness, unsteadiness, and sleepiness were noted usually after rapid increments of dosage from an initial dose of $25 \mathrm{mg}$. three times a day. Some patients were agitated after chlordiazepoxide and required a reduction in dosage. Ten of the 11 patients who received 300 to $600 \mathrm{mg}$. of chlordiazepoxide daily for several months showed new symptoms and signs of drug withdrawal when switched abruptly to placebos. Depression, agitation, insomnia, loss of appetite, nausea, aggravation of the psychosis, and grand mal seizures were noted 2 to 8 days after drug withdrawal. Minor electroencephalographic abnormalities (primarily slowing) were produced by chronic administration of large doses. The prolonged plasma halflife of this agent ( 48 hours after abrupt withdrawal) correlates with the relative slow onset of withdrawal symptoms.

Untoward effects and toxicity. Zbinden and colleagues ${ }^{335}$ summarized the experimental and clinical toxicology of chlordiazepoxide. In most of the 22 patients who took the drug with suicidal intent, single doses up to $2,250 \mathrm{mg}$. caused sedation, ataxia, and dysarthria. In a few instances, sleep and coma were noted. One healthy male volunteer ingested $50 \mathrm{mg}$. on the first day, $150 \mathrm{mg}$. on the second day, $500 \mathrm{mg}$. on the third day, and $1,000 \mathrm{mg}$. daily for another 9 days. The subject exhibited euphoria, loss of equilibrium, and difficulty concentrating after the third day. Following the very large doses ( 500 to $1,000 \mathrm{mg}$. per day), the subject was euphoric most of the time; later, he was hostile and irritable. Near the end of the experiment, he was ataxic and dysarthric. The symptoms disappeared progressively over about 5 days after cessation of medication. No signs of withdrawal were noted.

It has been repeatedly observed in many of the patients who attempted suicide with chlordiazepoxide that although they fell asleep, they could easily be aroused and were able to talk, eat, and drink. In the animal studies as well, the induced sleep was not deep. Single or repeated doses of chlordiazepoxide did not markedly affect arterial blood pressure, response to epinephrine or serotonin, or respiration. In a case of attempted suicide, marked vagotonia was noted which ceased after administration of atropine. Zbinden and colleagues were able to show in mice a synergistic effect between chlordiazepoxide and phenobarbital. The hypnotic effects of chloral hydrate, methyprylon, glutethimide, and ethyl alcohol were moderately or slightly enhanced. Clinically, there are few data available on the effects of such combinations. Of 22 patients who attempted to commit suicide, only 4 patients who took chlordiazepoxide in addition to high doses of methyprylon, imipramine, ethchlorvynol, and promazine plus alcohol did not show excessive sedation or coma. Obviously, much more data are necessary to determine whether the effects in animals or man are merely additive, synergistic, or due to true potentiation. In animal experiments, various analeptic agents were effective in counteracting the depressant effects of chlordiazepoxide. These included megimide, pentylenetetrazol, DL-amphetamine, and caffeine. Of considerable clinical importance is the observation that gastric lavage did not apparently markedly change the clinical picture of chlordiazepoxide intoxication because the drug appears in the blood within a few minutes, and peak levels occur in 2 hours after oral ingestion. Nevertheless, it would appear to be good clinical practice to perform gastric lavage as soon as possible even though much of the drug may already be absorbed. Lemere $^{201}$ reported that 4 patients given doses of chlordiazepoxide in excess of 80 mg. per day had behavioral intoxication with cumulative effect as suggested by the gradual onset of intoxication and the prolonged period ( 3 to 5 days) required for recovery following cessation of medication. Miller ${ }^{24}$ has emphasized the importance of behavioral toxicity as a side reaction to chlordiazepoxide and other psychoactive drugs. In a double blind study using 20 
mg. of chlordiazepoxide and matching placebos given daily for 1 week to normal male subjects, significant interference with judgment and vision in a simulated driving test were noted with the active drug.

Whitelaw $^{329}$ reported that chlordiazepoxide produced menstrual irregularities in 12 of 17 female infertility patients with previously normal cycles. A delay or failure to ovulate with premature or delayed menstruation was noted. The author suggested that this effect was similar to other tranquilizing agents.

One possible untoward effect of chlordiazepoxide which merits further attention is its role in causing agranulocytosis. Livingston, Pauli, and Murphy ${ }^{204}$ reported that 1 patient on trimethadione and chlordiazepoxide developed fulminating agranulocytosis that resulted in death. Chlordiazepoxide alone also may produce agranulocytosis, ${ }^{161}$ however the patient observed recovered upon withdrawal of the drug.

\section{References}

1. Abood, L. G.: Effect of chlorpromazine on phosphorylation of brain mitochondria, Proc. Soc. Exper. Biol. \& Med. 88:688-690, 1955.

2. Ader, R., and Clink, D. W.: Effects of chlorpromazine on the acquisition and extinction of an avoidance response in the rat, J. Pharmacol. \& Exper. Therap. 121:144-148, 1957.

3. Agranoff, B. W., Bradley, R. M., and Axelrod, J.: Determination and physiologic disposition of meprobamate, Proc. Soc. Exper. Biol. \& Med. 96:261-264, 1957.

4. Albaum, H. G.: The effect of central nervous system drugs on the enzyme activity of rat brain, U. S. Air Force, School of Aviation Medicine, Research Project 60-86, November, 1960 , pp. 1-8.

5. Alexander, L.: Effects of psychotropic drugs on* conditioned responses in man, in Rothlin, E., editor: Neuropsychopharmacology, New York, 1961, Elsevier Publishing Co., vol. 2, pp. 93-123.

6. Alexander, L., and Horner, S. R.: The effect of drugs on the conditional psychogalvanic reflex in man, J. Neuropsychiat. 2:246-261, 1961.

7. Allgén, L. G., Ekman, L., Reio, L., and Ullberg, S.: Biological fate of S $^{35}$-labelled Secergan, a quaternary phenothiazine compound, Arch. internat. pharmacodyn. 126:1-16, 1960.
8. Ansell, G. B., and Dohmen, H.: The depression of phospholipid turnover in brain tissue by chlorpromazine, J. Neurochem. 1:150-152, 1956.

9. Axelrod, J., Whitby, L. G., and Hertting, G.: Effect of psychotropic drugs on the uptake of $\mathrm{H}^{3}$-norepinephrine by tissues, Science 133: 383-384, 1961.

10. Ayd, F. J., Jr.: Current status of major tranquilizers, J. M. Soc. New Jersey 57:4-14, 1960.

11. Ayd, F. J., Jr.: A survey of drug-induced extrapyramidal reaction, J.A.M.A. 175:1054$1060,1961$.

12. Ayd, F. J., Jr.: Phenothiazine tranquilizers: Eight years of development, M. Clin. North America 45:1027-1040, 1961.

13. Balzer, H., Holtz, P., and Palm, D.: Reserpin und $\gamma$-amino-buttersäuregehalt des Gehirns, Experientia 17:38-40, 1961.

14. Barker, J. C., and Kerr, E. M.: Overdose of trifluoperazine, Lancet 2:1304, 1960.

15. Barr, M. N.: Effect of chlorpromazine on muscle spasm in human tetanus, Lancet 1: 991-993, 1958.

16. Barraclough, C. A.: Blockade of the release of pituitary gonadotrophin by reserpine, Fed. Proc. 14:9-10, 1955.

17. Barraclough, C. A.: Induction of pseudopregnancy in the rat by reserpine and chlorpromazine, Anat. Rec. 127:262, 1957.

18. Basmajian, J. V., and Szatmari, A.: Chlorpromazine and human spasticity: An electromyographic study, Neurology 5:856-860, 1955.

19. Basmajian, J. V., and Szatmari, A.: Effect of Largactil (chlorpromazine) on human spasticity and electromyogram, A.M.A. Arch. Neurol. \& Psychiat. 73:224-231, 1955.

20. Bastos, F. O., Carvalho, H. M., and Arantes, C. R. M.: Agranulocytosis during treatment with high doses of chlorpromazine: Report of case with recovery and review of literature, Rev. Hosp. clín. 14:300-311, 1959. (Abtract: J.A.M.A. 172:1336, 1960.)

21. Batterman, R. C., Grossman, A. J., Leifer, P., and Mouratoff, G. J.: Clinical re-evaluation of daytime sedatives, Postgrad. Med. 26:502$509,1959$.

22. Behn, W., Frahm, M., and Fretwurst, E.: Úber den diaplacentaren Úbergang von Phenothiazin-Derivaten, Klin. Wchnschr. 34:872, 1956.

23. Bein, H. J.: The pharmacology of Rauwolfia, Pharmacol. Rev. 8:435-483, 1956.

24. Benjamin, F. B., Ikai, K., and Clare, H. E.: Effect of proclorperazine on psychologic, psychomotor, and muscular performance, U. S. Armed Forces M. J. 8:1433-1440, 1957.

25. Benjamin, F. B., Ikai, K., and Clare, H. E.: 
Effect of a tranquilizing agent on galvanic skin response, J. Appl. Physiol. 11:216-218, 1957.

26. Bennett, J. L., and Kooi, K. A.: Five phenothiazine derivatives: Evaluation and toxicity studies, A.M.A. Arch. Gen. Psychiat. 4:413418, 1961.

27. Berger, F. M.: The pharmacological properties of 2-methyl-2-n-propyl-1,3-propanediol dicarbamate (Miltown), a new interneuronal blocking agent, J. Pharmacol. \& Exper. Therap. 112:413-423, 1954.

28. Berger, F. M.: The chemistry and mode of action of tranquilizing drugs, Ann. New York Acad. Sc. 67:685-700, 1957.

29. Berger, M.: Metabolic reactivity of brain and liver mitochondria towards chlorpromazine, J. Neurochem. 2:30-36, 1957.

30. Berger, M., Strecker, H. J., and Waelsch, H.: Action of chlorpromazine on oxidative phosphorylation of liver and brain mitochondria, Nature, London 177:1234-1235, 1956.

31. Bernsohn, J., Namajuska, I., and Cochrane, L. S. G.: Inhibition of brain cytochrome oxidase and ATP-ase by chlorpromazine analogues, Proc. Soc. Exper. Biol. \& Med. 92: 201-203, 1956.

32. Berti, T.: Struttura e distribuzione nell 'organismo di aminoderivati fenotiazinici, Farmaco, ed. sc. 9:374-378, 1954.

33. Bhattacharya, B. K., and Lewis, G. P.: The effects of reserpine and compound $48 / 80$ on the release of amines from the mast cells of rats, Brit. J. Pharmacol. 11:411-416, 1956.

34. Bogen-Tietz, E., Powelson, K. D., Weber, J., and Wildhaber, J.: Some effects of chlorpromazine on electrically induced convulsions in man, Confinia neurol. 18:57-63, 1958.

35. Bokonjic, N., and Trojaborg, W.: The effect of meprobamate on the electroencephalogram, during treatment, intoxication and after abrupt withdrawal, Electroencephalog. \& Clin. Neurophysiol. 12:177-184, 1960.

36. Bonafede, V. I.: Chlorpromazine (Thorazine) treatment of disturbed epileptic patients, A.M.A. Arch. Neurol. \& Psychiat. 77:243-246, 1957.

37. Boyd, E. M.: Chlorpromazine tolerance and physical dependence, J. Pharmacol. \& Exper. Therap. 128:75-78, 1960.

38. Boyd, L. J., Cammer, L., Mulinos, M. G., Huppert, V. F., and Hammer, H.: Meprobamate addiction, J.A.M.A. 168:1839-1843, 1958.

39. Brady, J. P., Nurnberger, J. I., and Tausig, T. N.: Experimental analysis of therapeutic variables by operant conditioning techniques, in Wortis, J., editor: Recent advances in biological psychiatry, New York, 1961, Grune \& Stratton, Inc., vol. 3, pp. 79-90.
40. Brauchitsch, H.: Endokrinologische Aspekte des Wirkungsmechanismus neuroplegischer Medikamente, Psychopharmacologia 2:1-21, 1961.

41. Braun, M.: Reserpine as a therapeutic agent in schizophrenia, Am. J. Psychiat. 116:744$745,1960$.

42. Brodie, B. B.: Effects of chlorpromazine, reserpine and monoamine oxidase inhibitors on the cardiovascular system by interaction with central and peripheral neurohumoral agents, in Skelton, F. R., editor: Hypertension, New York, 1959, American Heart Association, vol. VII, pp. 82-99.

43. Brodie, B. B., Maickel, R. P., and Westermann, E. O.: Action of reserpine on pituitary adrenocortical system through possible action on hypothalamus, in Kety, S. S., and Elkes, J., editors: Regional neurochemistry, New York, 1961, Pergamon Press, Inc., pp. 351361.

44. Brodie, B. B., Olin, J. S., Kuntzman, R., and Shore, P. A.: Possible interrelationship between release of brain norepinephrine and serotonin by reserpine, Science 125:1293, 1957.

45. Brodie, B. B., Prockop, D. J., and Shore, P. A.: An interpretation of the action of psychotropic drugs, Postgrad. Med. 24:296, 1958.

46. Brodie, B. B., and Shore, P. A.: A concept for a role of serotonin and norepinephrine as chemical mediators in the brain, Ann. New York Acad. Sc. 66:631, 1957.

47. Brodie, B. B., Spector, S., Kuntzman, R., and Shore, P. A.: Rapid biosynthesis of brain serotonin before and after reserpine administration, Naturwissenschaften 45:243, 1958.

48. Brodie, B. B., Tomich, E. G., Kuntzman, R., and Shore, P. H.: On the mechanism of action of reserpine-Effect of reserpine on capacity of tissues to bind serotonin, J. Pharmacol. \& Exper. Therap. 119:461, 1957.

49. Brooks, G. W.: Withdrawal from neuroleptic drugs, Am. J. Psychiat. 115:931-932, 1959.

50. Brüne, G. G., and Himwich, H. E.: Biphasic action of reserpine and isocarboxazid on behavior and serotonin metabolism, Science 133: 190-192, 1961.

51. Brüne, G. G., and Himwich, H. E.: Correlations between behavior and urinary indole amines during treatment with reserpine and isocarboxazid, separately and together, in Rothlin, E., editor: Amsterdam, 1961, Neuropsychopharmacology, Elsevier Publishing Co., vol. II, pp. 465-474.

52. Budnick, I. S., Leikin, S., and Hoeck, L. E.: Effect in the newborn infant of reserpine administered ante partum, A.M.A. J. Dis. Child. 90:286-289, 1955.

53. Bulla, J. D., II, Ewing, J. A., and Buffaloe, 
W. J.: Further controlled studies of meprobamate, Am. Pract. \& Digest Treat. 10:196I1964, 1959.

54. Burge, E.: Einfluss von Tranquillizer-Substanzen auf die Alkoholwirkung, Hefte Unfallheilk. 24:99-102, 1961.

55. Burton, R. M., Kaplan, N. O., Goldin, A., Leitenberg, M., Humphreys, S. R., and Sodd, M. A.: Effect of reserpine and promazine on diphosphopyridine nucleotide synthesis in liver, Science 127:30-32, 1958.

56. Carlsson, A., Rosengren, E., Bertler, A., and Nilsson, J.: Effect of reserpine on the metabolism of catechol amines, in Garattini, S., and Ghetti, V., editors: Psychotropic drugs, New York, 1957, Elsevier Publishing Co., pp. 363372.

57. Casey, J. F., Bennett, I. F., Lindley, C. J., Hollister, L. E., Gordon, M. H., and Springer, N. N.: Drug therapy in schizophrenia; a controlled study of the relative effectiveness of chlorpromazine, promazine, phenobarbital and placebo, A.M.A. Arch. Gen. Psychiat. 2:210$220,1960$.

58. Chicoine, L.: L'intoxication par les dérivés de la phénothiazine: Revue et rapport de 25 cas, Union méd. Canada 90:469-474, 1961.

59. Christensen, J., and Wase, A. W.: Distribution of $S^{35}$ in the mouse after administration of $\mathrm{S}^{35} 10$ (dimethylaminopropyl)-2-chlorophenothiazine (chlorpromazine), Acta pharmacol. et toxicol. 12:81-84, 1956.

60. Clark, M. L., and Johnson, P. C.: Amenorrhea and elevated level of serum cholesterol produced by a trifluoromethylated phenothiazine (SKF-5354-A), J. Clin. Endocrinol. 20: 641-646, 1960.

61. Claus, J. L.: A study of the localization of the site of action of central nervous system depressants and convulsants, Thesis, University of Houston, 1955.

62. Cohen, A. M.: Diuretic effect of chlorpromazine in patients with congestive heart failure, Am. Heart J. 54:907-914, 1957.

63. Cohen, I. M.: Complications of chlorpromazine therapy, Am. J. Psychiat. 113:115-121, 1956.

64. Conney, A. H., and Burns, J. J.: Biochemical pharmacological considerations of zoxazolamine and chlorzoxazone metabolism, Ann. New York Acad. Sc. 86:167-177, 1960.

65. Conney, A. H., Davison, C., Gastel, R., and Burns, J. J.: Adaptive increases in drug-metabolizing enzymes induced by phenobarbital and other drugs, J. Pharmacol. \& Exper. Therap. 130:1-8, 1960.

66. Courvoisier, S., Fournel, J., Ducrot, R., Kolsky, M., and Koetschet, P.: Propriétés pharmacodynamiques du chlorhydrate de chloro-3(diméthylamino-3'propyl)-10 phéno- thiazine (4.560 R. P.): Etude expérimentale d'un nouveau corps utilisé dans l'anesthésie potentialisée et dans l'hibernation artificielle, Arch. internat. pharmacodyn. 92:305-361, 1953.

67. Cutler, R. P., Monroe, J. J., and Anderson, T. E.: Effect of "tranquilizers" upon pathological activity in psychotic patients. I. Chlorpromazine, A.M.A. Arch. Neurol. \& Psychiat. 77:616-622, 1957.

68. Danhof, I. E.: Factors in the etiology of chlorpromazine jaundice: An experimental study, Texas Rep. Biol. \& Med. 16:443-457, 1958.

69. Darvill, F. T., Jr.: SU-3118 (carbethoxysyringoyl methylreserpate): A placebo study of antihypertensive potency and of side effects, Antibiotic Med. 5:598-603, 1958.

70. Dasgupta, S. R.: The effect of chlorpromazine on antidiuresis produced by 5-hydroxytryptamine (serotonin) in rats, Arch. internat. pharmacodyn. 112:264-271, 1957.

71. David, M., Benda, P., and Klein, F.: Traitement de l'état de mal épileptique par la chlorpromazine, Bull. et mém. Soc. med. Paris 69: 691-697, 1953.

72. Dawkins, M. J. R., Judah, J. D., and Rees, K. R.: The effect of chlorpromazine on the respiratory chain, Biochem. J. 72:204-209, 1959.

73. De Feo, V. J.: Effect of large doses of reserpine on the deciduoma response, Anat. Rec. 127:409, 1957.

74. De Feo, V. J., and Reynolds, S.R.M.: Modification of the menstrual cycle in rhesus monkeys by reserpine, Science 124:726-727, 1956.

75. Dennison, A. D., Jr., White, P. T., Moore, R. B., and Pierce, W. J.: Effect of reserpine upon the human electroencephalogram, Neurology 5:56-57, 1955.

76. De Ropp, R. S., and Snedeker, E. H.: Effects of drugs on amino acid levels in brain: Excitants and depressants, Proc. Soc. Exper. Biol. \& Med. 106:696-700, 1961.

77. Dickel, H. A., Dixon, H. H., Shanklin, J. G., and Dixon, H. H., Jr.: Choosing a drug for the anxious working patient, Scientific Exhibit at American Medical Association, Denver Clinical Meeting, Nov. 26 to 30, 1961.

78. DiMascio, A., Havens, L. L., and Snell, J. E.: A comparison of four phenothiazine derivatives: A preliminary report on the assessment of chlorpromazine, promethazine, perphenazine, and trifluoperazine, in Wortis, J., editor: Recent advances in biological psychiatry, New York, 1961, Grune \& Stratton, Inc., vol. III, pp. 68-76.

79. DiMascio, A., Klerman, G. L., Rinkel, M., Greenblatt, M., and Brown, J.: Psychophysiologic evaluation of phenyltoloxamine, a new 
phrenotropic agent, Am. J. Psychiat. 115: 301-317, 1958.

80. Dobkin, A. B.: Potentiation of thiopental anesthesia by derivatives and analogues of phenothiazine, Anesthesiology 21:292-296, 1960.

81. Dobkin, A. B., Gilbert, R. G. B., and Lamoureaux, L.: Physiological effects of chlorpromazine, Anaesthesia 9:157-174, 1954.

82. Dobkin, A. B., and Palko, D.: The antisialagogue effect of phenothiazine derivatives: Comparison of promazine, levomepromazine, trifluoperazine, proclorperazine, methdilazine and prothipendyl, Anesthesiology 21:260-262, 1960.

83. Domino, E. F.: Sites of action of some central nervous system depressants, Ann. Rev. Pharmacol. 2:215-250, 1962.

84. Domino, E. F., and Rech, R. H.: Observations on the initial hypertensive response to reserpine, J. Pharmacol. \& Exper. Therap. 121:171-182, 1957.

85. Doughty, R.: The incidence of jaundice associated with Thorazine therapy, Philadelphia, 1955, Smith, Kline \& French Laboratories.

86. Dreyfuss, F.: Jaundice due to chlorpromazine, J.A.M.A. 168:2044, 1958.

87. Duff, R. S., and Ginsburg, J.: Antagonism between chlorpromazine and noradrenaline in blood vessels of the hands, Brit. J. Pharmacol. 11:318-322, 1956.

88. Egdahl, R. H., Richards, J. B., and Hume, D. M.: Effect of reserpine on adrenocortical function of unanesthetized dogs, Science 123: 418, 1956.

89. Eggers, G. W. N., Jr., Corssen, G., and Allen, C. R.: Comparison of vasopressor responses in the presence of phenothiazine derivatives, Anesthesiology 20:261-267, 1959.

90. Eiduson, S., Geller, E., and Cohen, S.: The excretion and metabolism of $S^{35}$-labeled thioridazine in humans, in Transactions of the Sixth Research Conference on Cooperative Chemotherapy Studies in Psychiatry and Broad Research Approaches to Mental Illness, Cincinnati, Ohio, March 27-29, 1961, Washington, D. C., 1961, Veterans Administration Department of Medicine and Surgery, pp. 7580.

91. Emmerson, J. L., Miya, T. S., and Yim, G. K. W.: The distribution and metabolic state of carbon-14 meprobamate in the rat brain, J. Pharmacol. \& Exper. Therap. 129:89-93, 1960.

92. Ernsting, M. J. E., Kafoe, W. F., Nauta, W. Th., Oosterhuis, H. K., and deWaart, C.: Biochemical studies on psychotropic drugs. I. The effect of psychotropic drugs on $\gamma$-aminobutyric acid and glutamic acid in brain tissues, J. Neurochem. 5:121-127, 1960.
93. Essig, C. F.: Withdrawal convulsions in dogs following chronic meprobamate intoxication, A.M.A. Arch. Neurol. \& Psychiat. 80:414$417,1958$.

94. von Euler, U. S., and Lishajko, F.: Effect of some drugs on noradrenaline release from nerve granules, Biochem. Pharmacol. 8:62, 1961.

95. Fernandes, B., and Leitão, G.: Incidents and accidents in chlorpromazine therapy, J. Clin. \& Exper. Psychopath. 17:70-76, 1956.

96. Finger, K. F., Weisman, A., and Tretter, J. R.: Behavioral and biochemical activity of a new series of benzoquinolizine derivatives, Pharmacologist 3:75, 1961.

97. Forrest, F. M., and Forrest, I. S.: Urine tests for the detection of the newer phenothiazine compounds, drug excretion rates, clinical implications and recent developments in research on phenothiazine drug, in Transactions of the Fourth Research Conference on Cooperative Chemotherapy Studies in Psychiatry and Research Approaches to Mental Illness, Memphis, Tenn., May 20-22, 1959, Washington, D. C., 1960, Veterans Administration Department of Medicine and Surgery, pp. 245-247.

98. Forrest, F. M., Forrest, I. S., and Mason, A. S.: Review of rapid urine tests for phenothiazine and related drugs, Am. J. Psychiat. 118:300-307, 1961.

99. Forrest, I. S., and Forrest, F. M.: Metabolism and excretion of phenothiazine drugs, in Transactions of the Fifth Research Conference on Cooperative Chemotherapy Studies in Psychiatry and Research Approaches to Mental Illness, Cincinnati, Ohio, June 6 to 8, 1960, Washington, D. C., 1960, Veterans Administration Department of Medicine and Surgery, pp. 128-131.

100. Foster, C. A., O'Mullane, E. J., Caskell, P., and Churchill-Davidson, H. C.: Chlorpromazine: A study of its action on the circulation in man, Lancet 2:614-617, 1954.

101. Fraser, H. F., and Isbell, H.: Chlorpromazine and reserpine: a) effects of each, and of combinations of each with morphine, b) failure of each in treatment of acute abstinence from morphine, J. Pharmacol. \& Exper. Therap. 116:21, 1956.

102. Freedman, D. X., and Benton, A. J.: Persisting effects of reserpine in man, New England J. Med. 264:529-533, 1961.

103. Friend, D. G.: The tranquilizers, M. Clin. North America 42:1253-1268, 1958.

104. Friend, D. G.: The phenothiazines, Clin. Pharmacol. \& Therap. 1:5-10, 1960.

105. Fyodorov, N. A.: The fate of three phenothiazine compounds in the organism: Aminazine-S $S^{35}$ (chlorpromazine), promazine- $S^{35}$ and chlormepazine-S ${ }^{35}$ (chlorpakatal), in Pro- 
ceedings of the Second United Nations International Conference on the Peaceful Uses of Atomic Energy, Geneva, September 1-13, 1958, Geneva, 1958, United Nations, vol. 24, pp. 205-215.

106. Gal, E. M., Drewes, P. A., and Barraclough, C. A.: Effect of reserpine and the metabolism of serotonin in tryptophan deficient rats, Biochem. Pharmacol. 8:32, 1961.

107. Gaunt, R., Chart, J. J., and Renzi, A. A.: Endocrine pharmacology, Science 133:613$621,1961$.

108. Gaunt, R., Renzi, A. A., Antonchak, M., Miller, G. J., and Gilman, M.: Endocrine aspects of the pharmacology of reserpine, Ann. New York Acad. Sc. 59:22-35, 1954.

109. Gey, K. F., and Pletscher, A.: Influence of chlorpromazine and chlorprothixene on the cerebral metabolism of 5-hydroxytryptamine, norepinephrine and dopamine, J. Pharmacol. \& Exper. Therap. 133:18-24, 1961.

110. Ginsburg, J., and Duff, R. S.: Effect of chlorpromazine on adrenaline vasoconstriction in man, Brit. J. Pharmacol. 11:180-185, 1956.

111. Glaviano, V. V., and Wang, S. C.: Dual mechanism of the antiemetic action of chlorpromazine, Fed. Proc. 13:358, 1954.

112. Glotfelty, J. S., and Wilson, W. P.: Effects of tranquilizing drugs on reticular system activity in man, North Carolina M. J. 17:401$405,1956$.

113. Goldenberg, H., and Fishman, V.: Species dependence of chlorpromazine metabolism, Proc. Soc. Exper. Biol. \& Med. 108:178-182, 1961.

114. Goldman, D.: The major complications of treatment of psychotic states with chlorpromazine and reserpine and their management, Psychiat. Res. Rep. 4:79-88, 1956.

115. Goldman, D.: Parkinsonism and related phenomena from administration of drugs: Their production and control under clinical conditions and possible relation to therapeutic effect, Rev. canad. biol. 20:549-560, 1961.

116. Goldsmith, R. W.: Antidote for proclorperazine intoxication in children, J.A.M.A. 172: $361,1959$.

117. Gordon, M.: Psychopharmacological agents, in Burger, A., editor: Medicinal chemistry, New York, 1960, Interscience Publishers, Inc., pp. 397-418.

118. Green, J. P., Paasonen, M. K., and Giarman, N. J.: Blood 5-hydroxytryptamine (serotonin) levels after reserpine and electroshock therapy, Proc. Soc. Exper. Biol. \& Med. 94:428$430,1957$.

119. Grenell, R. G., Mendelson, J., and McElroy, W. D.: Effects of chlorpromazine on metabolism in central nervous system, A.M.A. Arch. Neurol. \& Psychiat. 73:347-351, 1955.
120. Gros, H., Peterfalvi, M., and Jequier, R.: Exploration à toutes doses d'un dérivé nonsédatif da la réserpine, le R-694, dans le traitement de l'hypertension artérielle, Algérie méd. 63:297-298, 1959.

121. Gross, M., Hitchman, I. L., Reeves, W. P., Lawrence, J., and Newell, P. C.: Discontinuation of treatment with ataractic drugs, in Wortis, J., editor: Recent advances in biological psychiatry, New York, 1961, Grune \& Stratton, Inc., vol. III, p. 44-67.

122. Gupta, S. K., Patel, M. A., and Joseph, A. D.: Effects of chlorpromazine and epinephrine on blood-sugar of rabbits, Arch. internat. pharmacodyn. 128:82-88, 1960.

123. Guth, P. S., and Spirtes, M. A.: Mode of action of chlorpromazine, Biochem. Pharmacol. 8:170, 1961.

124. Haizlip, T. M., and Ewing, J. A.: Meprobamate habituation: A controlled clinical study, New England J. Med. 258:1181-1186, 1958.

125. Hansson, E., and Schmiterlöw, C. G.: A comparison of the distribution, excretion and metabolism of a tertiary (promethazine) and a quaternary (aprobit) phenothiazine compound labelled with $\mathrm{S}^{35}$, Arch. internat. pharmacodyn. 131:309-324, 1961.

126. Hardisty, R. M., Ingram, G. I. C., and Stacey, R. S.: Reserpine and human platelet 5hydroxytryptamine, Experientia 12:424, 1956.

127. Harris, A. F., Saifer, A., and Volk, B. W.: Interaction of chlorpromazine with strandin, Proc. Soc. Exper. Biol. \& Med. 104:542-547, 1960.

128. Harwood, C. T., and Mason, J. W.: Acute effects of tranquilizing drugs on the anterior pituitary-ACTH mechanism, Endocrinology 60:239-246, 1957.

129. Haverback, B. J., Dutcher, T. F., Shore, P. A., Tomich, E. G., Terry, L. L., and Brodie, B. B.: Serotonin changes in platelets and brain induced by small daily doses of reserpine: Lack of depletion of platelet serotonin on hemostatic mechanisms, New England J. Med. 256:343-345, 1957.

130. Haverback, B. J., Hogben, A. M., Moran, N. C., and Terry, L. L.: Effect of serotonin (5hydroxytryptamine) and related compounds on gastric secretion and intestinal motility in the dog, Gastroenterology 32:1058, 1957.

131. Haverback, B. J., Stevenson, - T. D., Sjoerdsma, A., and Terry, L. L.: The effects of reserpine and chlorpromazine on gastric secretion, Am. J. M. Sc. 230:601, 1955.

132. Haynes, E. E.: Urinary excretion of chlorpromazine in man, J. Lab. \& Clin. Med. 56: 570-575, 1960.

133. Helper, E. W., Carver, M. J., Jacobi, H. P., and Smith, J. A.: The effect of tranquilizing 
agents and related compounds on the succinoxidase system, Arch. Biochem. \& Biophys. 76:354-361, 1958 .

134. Henatsch, H. D., and Ingvar, D. H.: Chlorpromazin und Spastizität: Eine experimentelle elektrophysiologische Untersuchung, Arch. Psychiat. 195:77-93, 1956.

135. Hertz, A.: Drugs and the conditioned avoid. ance response, Internat. Rev. Neurobiol. 2: $229-277,1960$.

136. Hess, S. M., Shore, P. A., and Brodie, B. B.: Persistence of reserpine action after the disappearance of drug from brain: Effect of serotonin, J. Pharmacol. \& Exper. Therap. 118:84-89, 1956.

137. Hetzel, C. A.: Method for the estimation of phenothiazine derivatives in urine and blood, Clin. Chem. 7:130-135, 1961.

138. Himwich, H. E., Rinaldi, F., and Willis, D.: An examination of phenothiazine derivatives with comparisons of their effects on the alerting reaction, chemical structure and therapeutic efficacy, J. Nerv. \& Ment. Dis. 124: 53-57, 1956.

139. Holliday, A. R., and Dille, J. M.: The effects of meprobamate, chlorpromazine, pentobarbital and a placebo on a behavioral task performed under stress conditions, J. Comp. \& Physiol. Psychol. 51:811-815, 1958.

140. Hollister, L. E.: Complications from the use of tranquilizing drugs, New England J. Med. 257:170-177, 1957.

141. Hollister, L. E.: Complications from psychotherapeutic drugs. II, New England \}. Med. 264:345-347, 1961.

142. Hollister, L. E., and Barthel, C. A.: Changes in the electroencephalogram during chronic administration of tranquilizing drugs, Electroencephalog. \& Clin. Neurophysiol. I1:792$795,1959$.

143. Hollister, L. E., Caffey, E. M., Jr., and Klett, C. J.: Abnormal symptoms, signs and laboratory tests during treatment with phenothiazine derivatives, Clin. Pharmacol. \& THerap. 1:284-293, 1960.

144. Hollister, L. E., and Glazener, F. S.: Withdrawal reactions from meprobamate, alone and combined with promazine: Controlled study, Psychopharmacologia 1:336-341, 1960.

145. Hollister, L. E., Motzenbecker, F. P., and Degan, R. O.: Withdrawal reactions from chlordiazepoxide (Librium), Psychopharmacologia 2:63-68, 1961.

146. Hollister, L. E., Traub, L., and Prusmack, J. J.: Use of thioridazine for intensive treatment of schizophrenics refractory to other tranquilizing drugs, J. Neuropsychiat. 1:200$204,1960$.

147. Holzbauer, M., and Vogt, M.: Depression by reserpine of the noradrenaline concentration in the hypothalamus of the cat, J. Neurochem. 1:8, 1956.

148. Hooper, J. H., Jr., Welch, V. C., and Shackelford, R. T.: Abnormal lactation associated with tranquilizing drug therapy, J.A.M.A. 178:506-507, 1961.

149. Huang, C. L., and Kurland, A. A.: A quantitative study of chlorpromazine and its sulfoxides in the urine of psychotic patients, Am. J. Psychiat. 118:428-437, 1961.

150. Huang, C. L., Sands, F. L., and Kurland, A. A.: Paper chromatographic analysis of urinary chlorpromazine metabolites in psychotic patients, Clin. Chem. 7:573, 1961.

15l. Hudson, R. D., and Domino, E. F.: Evidence for a brainstem action of chlorpromazine on some motor reflexes, Fed. Proc. 20:307, 1961.

152. Hughes, F. B., and Brodie, B. B.: The mechanism of serotonin and catecholamine uptake by platelets, J. Pharmacol. \& Exper. Therap. 127:96-102, 1959.

153. Hughes, F. B., Shore, P. A., and Brodie, B. B.: Serotonin storage mechanism and it, interaction with reserpine, Experientia 14:178, 1958.

154. Irwin, S.: Factors influencing sensitivity to stimulant and depressant drugs affecting (a) locomotor and (b) conditioned avoidance behavior in animals, in Sarwer-Foner, G. J., editor: The dynamics of psychiatric drug therapy, Springfield, Ill., 1960, Charles C Thomas, Publisher, pp. 5-28.

155. Irwin, S.: Correlation in rats between the locomotor and avoidance suppressant potencies of eight phenothiazine tranquilizers, Arch. internat. pharmacodyn. 132:279-286, 1961.

156. Isbell, H., Altschul, S., Kornetsky, C. H., Eisenman, A. J., Flanary, H. G., and Fraser, H. F.: Chronic barbiturate intoxication: An experimental study, Arch. Neurol. \& Psychiat. 64:1-28, 1950 .

157. Jindal, M. N., Tiwari, N. M., and Kherdikar, P. R.: Structure activity relationship of chlorpromazine and related phenothiazine derivatives on pentobarbitone anesthesia in mice, Arch. internat. pharmacodyn. 129:166, 1960.

158. Jonsson, C. O., and Andersén, K.: Influence of emylcamate, meprobamate, and placebo on psychologic test performance, Clin. PharmaCOL. \& THERAP. 1:708-715, 1960.

159. Jörgensen, R. S., and Wulff, M. H.: The effect of orally administered chlorpromazine on the electroencephalogram of man, Electroencephalog. \& Clin. Neurophysiol. 10:325-329, 1958.

160. Joyce, C. R. B., Edgecombe, P. C. E., Kennard, D. A., Weatherall, M., and Woods, D. P.: Potentiation by phenobarbitone of effects of ethyl alcohol on human behavior, J. Ment. Sc. 105:51-60, 1959 . 
161. Kaelbling, R.: Agranulocytosis due to chlordiazepoxide hydrochloride, J.A.M.A. 174: 1863-1865, 1960.

162. Kähler, H. J.: Veränderungen des Blutbildes durch Derivate des Phenothiazins, Deutsche med. Wchnschr. 86:1001-1005, 1961.

163. Kamin, I., and Shaskan, D. A.: Death due to massive overdose of meprobamate, Am. J. Psychiat. 115:1123-1124, 1959.

164. Kato, R.: Reduced sensitivity to some drugs 48 hours after chlorpromazine treatment, Experientia 16:427-428, 1960.

165. Kato, R.: Induced increase of meprobamate metabolism in rats treated with phenobarbital or phenaglycodol, Med. exper. 3:95-100, 1960.

166. Kelly, E. L., Miller, J. G., Marquis, D, G., Gerard, R. W., and Uhr, L.: Personality differences and continued meprobamate and proclorperazine administration, A.M.A. Arch. Neurol. \& Psychiat. 80:241-246, 1958.

167. Kelly, E. L., Miller, J. G., Marquis, D. G., Gerard, R. W., and Uhr, L.: Continued meprobamate and proclorperazine administration and behavior, A.M.A. Arch. Neurol. \& Psychiat. 80:247-252, 1958.

168. Khauw, L. B.: The inhibition of alcohol dehydrogenase by chlorpromazine, Fed. Proc. 19:280, 1960.

169. Kinross-Wright, J.: Newer phenothiazine drugs in treatment of nervous disorders, J.A.M.A. 170:1283-1288, 1959.

170. Kinross-Wright, V.: Complications of chlorpromazine treatment, Dis. Nerv. System 16: 114-119, 1955.

171. Kivalo, E., Rinne, U. K., and Marjanen, P.: Diuretic effect of chlorpromazine with special references to the hypothalamic neurosecretory substance, Ann. med, exper. et biol. Fenniae 36:185, 1958.

172. Klerman, G. L., and DiMascio, A.: Psychological effects of piperazine phenothiazines, Fed. Proc. 20:393, 1961.

173. Klerman, G. L., DiMascio, A., Havens, L. L., and Snell, J. E.: Sedation and tranquilization: A comparison of the effects of a number of psychopharmacologic agents upon normal human subjects, A.M.A. Arch. Gen. Psychiat. 3: 4-13, 1960.

174. Klett, C. J., and Caffey, E. M., Jr.: Weight changes during treatment with phenothiazine derivatives, J. Neuropsychiat. 2:102, 1960.

175. Kline, N. S.: Use of Rauwolfia serpentina Benth. in neuropsychiatric conditions, Ann. New York Acad. Sc. 59:107-132, 1954.

176. Kline, N. S.: Clinical applications of reserpine, in Kline, N. S., editor: Psychopharmacology, Washington, D. C., 1956, American Association for the Advancement of Science, pp. 81-108.
177. Kline, N. S., Barsa, J., and Gosline, E.: Management of side effects of reserpine and combined reserpine-chlorpromazine treatment, Dis. Nerv. System 17:352-358, 1956.

178. Koechlin, B. A., and Schwartz, M. A.: The metabolic fate of chlordiazepoxide, Fed. Proc. 20:171, 1961.

179. Kohn, N., and Myerson, R. M.: Cholestatic hepatitis associated with trifluoperazine, New England J. Med. 264:549-550, 1961.

180. Kopera, J., and Armitage, A. K.: Comparison of some pharmacological properties of chlorpromazine, promethazine, and pethidine, Brit. J. Pharmacol. 9:392-401, 1954.

181. Kopmann, E., and Hughes, F. W.: Effects of glutamate on the potentiating action of certain ataraxics, Proc. Soc. Exper. Biol. \& Med. 97:83-85, 1958.

182. Kopmann, E., and Hughes, F. W.: Potentiating effect of alcohol on tranquilizers and other central depressants, A.M.A. Arch. Gen. Psychiat. 1:7-11, 1959.

183. Kornetsky, C.: Effects of meprobamate, phenobarbital and dextro-amphetamine on reaction time and learning in man, J. Pharmacol. \& Exper. Therap. 123:216-219, 1958.

184. Kornetsky, C.: Alterations in psychomotor functions and individual differences in responses produced by psychoactive drugs, in Uhr, L., and Miller, J. G., editors: Drugs and behavior, New York, 1960, John Wiley \& Sons, Inc., pp. 297-312.

185. Kornetsky, C., Pettit, M., Wynne, R., and Evarts, E. V.: A comparison of the psychological effects of acute and chronic administration of chlorpromazine and secobarbital (quinalbarbitone) in schizophrenic patients, J. Ment. Sc. 105:190-198, 1959.

186. Kornetsky, C., Vates, T. S., and Kessler, E. $\mathrm{K}$ : A comparison of hypnotic and residual psychological effects of single doses of chlorpromazine and secobarbital in man, J. Pharmacol. \& Exper. Therap. 127:51-54, 1959.

187. Korst, D. R.: Agranulocytosis caused by phenothiazine derivatives, J.A.M.A. 170: 2076-2081, 1959.

188. Kovács, K., Kovács, G. S., Kovács, B. M., and Petri, G.: The effect of chlorpromazine on the activity of the antidiuretic hormone, Arch. internat. pharmacodyn. 109:1-7, 1957.

189. Kris, E. B.: Effects of pharmacotherapy on work and learning ability-A five year follow-up study, in Wortis, J., editor: Recent advances in biological psychiatry, New York, 1961, Grune \& Stratton, Inc., vol. III, pp. 3034.

190. Lambros, V. S.: The use of reserpine in certain neurological disorders: Organic convulsive states, enuresis, and head injuries, Ann. New York Acad. Sc. 61:211-214, 1955. 
191. Lamphier, T. A., and Pine, H.: Emylcamate, a new tranquilizing and muscle relaxant agent, Psychosomatics 1:204-207, 1960.

192. Lanzoni, V., Li, T.-H., and Etsten, B.: Circulatory effects of intravenous proclorperazine (Compazine) in humans, Fed. Proc. 17:386, 1958.

193. Lasagna, L.: A study of hypnotic drugs in patients with chronic diseases: Comparative efficacy of placebo; methylprylon (Noludar); meprobamate (Miltown), (Equanil); pentobarbital; phenobarbital; secobarbital, J. Chron. Dis. 3:122-133, 1956.

194. Lasagna, L., and De Kornfeld, T. J.: Methotrimeprazine: a new phenothiazine derivative with analgesic properties, J.A.M.A. 178:887. $890,1961$.

195. Lasagna, L., and McCann, W. P.: Effect of "tranquilizing" drugs on amphetamine toxicity in aggregated mice, Science 125:1241-1242, 1957.

196. Laties, V. G.: Effects of meprobamate on fear and palmar sweating, J. Abnorm. \& Social Psychol. 59:156-161, 1959.

197. Laties, V. G., and Weiss, B.: A critical review of the efficacy of meprobamate (Miltown, Equanil) in the treatment of anxiety, J. Chron. Dis. 7:500-519, 1958.

198. Laurence, D. R., Berman, E., Scragg, J. N., and Adams, E. B.: A clinical trial of chlorpromazine against barbiturates in tetanus, Lancet 1:987-991, 1958.

199. Laurence, D. R., and Webster, R. A.: Tachyphylaxis to the antitetanus activity of some phenothiazine compounds, Brit. J. Pharmacol. 16:296-308, 1961.

200. Lehmann, H. E., and Csank, J.: Differential screening of phrenotropic agents in man: Psychophysiologic test data, J. Clin. \& Exper. Psychopath. 18:222-235, 1957.

201. Lemere, F.: Toxic reactions to chlordiazepoxide, J.A.M.A. 174:893, 1960.

202. Liberson, W. T.: Analysis of the effects of psychotropic drugs on rigid behavior in rodents, in Transactions of the Sixth Research Conference on Cooperative Chemotherapy Studies in Psychiatry and Broad Research Approaches to Mental Illness, Cincinnati, Ohio, March 27-29, 1961, Washington, D. C., 1961, Veterans Administration Department of Medicine and Surgery, pp. 159-166.

203. Lienert, G. A., and Traxel, W.: The effects of meprobamate and alcohol on galvanic skin responses, J. Psychol. 48:329, 1959.

204. Livingstone, S., Pauli, L., and Murphy, J. B.: Ineffectiveness of chlordiazepoxide hydrochloride in epilepsy, J.A.M.A. 177:243-244, 1961 .

205. Lomas, J., Boardman, R. H., and Markowe, M.: Complications of chlorpromazine therapy in 800 mental-hospital patients, Lancet $\mathbf{l}$ : 1144-1147, 1955.

206. Loomis, T. A., and West, T. C.: Comparative sedative effects of a barbiturate and some tranquilizer drugs on normal subjects, J. Pharmacol. \& Exper. Therap. 122:525-531, 1958.

207. Ludwig, B. J., Douglas, J. F., Powell, L. S., Meyer, M., and Berger, F. M.: Structures of the major metabolites of meprobamate, J. M. \& Pharmaceut. Chem. 3:53-64, 1961.

208. McGeer, P. L., Boulding, J. E., Gibson, W. C., and Foulkes, R. G.: Drug-induced extrapyramidal reactions: Treatment with diphenhydramine hydrochloride and dihydroxyphenylalanine, J.A.M.A. 177:665-670, 1961.

209. McGuire, T. F., and Leary, F. J.: Tranquilizing drugs and stress tolerance, Am. J. Pub. Health 48:578-584, 1958.

210. MacLean, P. D.: Hippocampal studies: Their possible bearing on neuropsychiatry, A.M.A. Arch. Neurol. \& Psychiat. 74:219-220, 1955.

211. McQuarrie, D. G., and Fingl, E.: Hyperexcitability in mice following cessation of chronic administration of ethanol, Fed. Proc. 14:369, 1955.

212. McQueen, E. G., Doyle, A. E., and Smirk, F. H.: Mechanism of hypotensive action of reserpine, an alkaloid of Rauwolfia serpentina, Nature, London 174:1015, 1954.

213. Magee, W. L., Berry, J. F., and Rossiter, R. J.: Effect of chlorpromazine and azacyclonal on the labelling of phosphatides in brain slices, Biochim. et biophys. acta 21:408-409, 1956.

214. Malhotra, C. L., and Das, P. K.: Effect of reserpine on the acetylcholine content of the heart, the ileum and the hypothalamus of the dog, Brit. J. Pharmacol. 18:190-193, 1962.

215. Malhotra, C. L., and Pundlik, P. G.: The effect of reserpine on the acetylcholine content of different areas of the nervous system of the dog, Brit. J. Pharmacol. 14:46-47, 1959.

216. Mariani, L., and Vertua, R.: Modificata fissazione "in vivo" di serotonina marcata in presenza di chlorpromazina, Minerva nucl. 5:90-93, 1961.

217. Marquis, D. G., Kelly, E. L., Miller, J. G., Gerard, R. W., and Rapaport, A.: Experimental studies of behavioral effects of meprobamate on normal subjects, Ann. New York, Acad. Sc. 67:701-711, 1957.

218. Marx, G. F.: Placental transfer and drugs used in anesthesia, Anesthesiology 22:294$313,1961$.

219. Mathe, V., and Kassay, G.: The carbolyydrate metabolism of the brain in various inhibitory conditions of the central nervous system, in Abstracts of Communcation, $V$ th International 
Congress of Biochemistry, Moscow, August 10-16, 1961, Oxford, 1961, Pergamon Press, Ltd., p. 216.

220. Maynert, E. W.: Metabolic fate of drugs, Ann. Rev. Pharmacol. 1:45-64, 1961.

221. Meier, R., Brüni, C., and Tripod, J.: Différenciation pharmacodynamique de l'aprésoline du serpasil et de la chlorpromazine lors de leur action sur la rétention hydriaque du rat, Arch. internat. pharmacodyn. 104:137-145, 1955.

222. Melander, B.: Psychotechnische Untersuchungen an Autofahrern unter Medikamenteneinwirkung, München. med. Wchnschr. 99: 1340-1342, 1957.

223. Menguy, R. B., Bollman, J. L., Grindlay, J. H., and Cain, J. B.: Biliary stasis produced by chlorpromazine: An experimental study, Proc. Staff Meet. Mayo Clin. 30:601-606, 1955.

224. Miller, J. G.: Objective measurements of the effects of drugs on driver behavior, J.A.M.A. 179:941-943, 1962.

225. Miner, R. W., editor: Reserpine (Serpasil) and other alkaloids of Rauwolfia serpentina: Chemistry, pharmacology, and clinical implications, Ann. New York Acad. Sc. 59:1-140, 1954.

226. Miner, R. W, editor: Reserpine in the treatment of neuropsychiatric, neurological and related problems, Ann. New York Acad. Sc. 61:1-280, 1955 .

227. Mirsky, A. F., Primac, D. W., and Bates, R.: The effects of chlorpromazine and secobarbital on the c.p.t., J. Nerv. \& Ment. Dis. 128: 12-17, 1959.

228. Mitchell, L. E.: The effects of ataratic therapy on conditioning rate of GSR in a group of psychiatric patients, Am. Psychologist 13: $343,1958$.

229. Monroe, R. R., Heath, R. G., Mickle, W. A., and Miller, W.: A comparison of cortical and subcortical brain waves in normal, barbiturate, reserpine, and chlorpromazine sleep, Ann. New York Acad. Sc. 61:56-71, 1955.

230. Moyer, J. H.: The pharmacology of chlorpromazine, J. Clin. \& Exper. Psychopath. 16: 179-190, 1955.

231. Moyer, J. H., Kent, B., Knight, R., Morris, G., Huggins, R, and Handley, C. A.: Laboratory and clinical observations on chlorpromazine (SKF-2601-A)-hemodynamic and toxicological studies, Am. J. M. Sc. 227:283$290,1954$.

232. Moyer, J. H., Pevey, K., Heider, C. H., and Kinross-Wright, V.: A comparative study of four tranquilizing agents, phenobarbital, and inert placebo, Geriatrics 13:153-170, 1958.

233. Müeller, J. M., Schlittler, E., and Bein, H. J.: Reserpin, der sedative Wirkstoff aus Rau- wolfia serpentina Benth., Experientia 8:338, 1952.

234. Muscholl, E., and Vogt, M.: The action of reserpine on peripheral sympathetic ganglia, J. Physiol. 141:132, 1958.

235. Noce, R. H., Williams, D. B., and Rapaport, W.: Reserpine (Serpasil) in the management of the mentally ill, J.A.M.A. 158:11-15, 1955.

236. Nodine, J. H.: Comparison of tranquilizers by human bioassay, Clin. Res. 9:236, 1961.

237. Nyquist, R. H., Comarr, A. E., and Bors, E.: Comparative study of antispasmodic drugs in patients with spinal cord injuries, Arch. Phys. Med. 39:683-691, 1958.

238. Orlans, F. B. H., Finger, K. F., and Brodie, B. B.: Pharmacological consequences of the selective release of peripheral norepinephrine by syrosingopine (SU 3118), J. Pharmacol. \& Exper. Therap. 128:131-139, 1960.

239. Paasonen, M. K., and Krayer, O.: The release of norepinephrine from the mammalian heart by reserpine, J. Pharmacol. \& Exper. Therap. 123:153-160, 1958.

240. Palm, D., Balzer, H., and Holtz, P.: Reserpin und $\gamma$-amino-buttersäuregehalt des Gehirns, Arch. exper. Path. u. Pharmakol. 241:190$191,1960$.

241. Palm, D., Balzer, H., and Holtz, P.: Amino acid and carbohydrate metabolism of brain after reserpine, Biochem. Pharmacol. 8:34, 1961.

242. Parrish, A. E., and Levine, E. H.: Chlorpromazine-induced diuresis, J. Lab. \& Clin. Med. 48:264-269, 1956.

243. Paul-David, J. C., and Unna, K. R.: Quantification of the effects of phenothiazine derivatives on the EEG activation response, Pharmacologist 2:79, 1960 .

244. Pelikan, E. W., and Kensler, C. J.: Sedatives: Their pharmacology and uses, M. Clin. N. America 42:1217-1237, 1958.

245. Perlstein, M. A., Use of meprobamate (Miltown) in convulsive and related disorders, J.A.M.A. 161: 1040-1044, 1956.

246. Perlstein, M. A., Stein, M. D., and Elam, H.: Routine treatment of tetanus, J.A.M.A. 173: 1536-1541, 1960.

247. Perlstein, M. A., Turner, M., and Elam, H.: Electromyographic observations in patients with tetanus: With special reference to the effect of drugs, Arch. Phys. Med. 39:283$289,1958$.

248. Pfeiffer, C. C., Riopelle, A. J., Smith, R. P., Jenney, E. H., and Williams, H. L.: Comparative study of the effect of meprobamate on the conditioned response on strychnine and pentylenetetrazol thresholds on the normal electroencephalogram, and on polysynaptic reflexes, Ann. New York Acad. Sc. 67: 734-745, 1957. 
249. Phillips, B. M., Miya, T. S., and Yim, G. K. W.: Studies on the mechanism of meprobamate tolerance in the rat, J. Pharmacol. \& Exper. Therap. 135:223-229, 1962.

250. Pisciotta, A. V., Kohldahl, J., and Mally, M. J.: Effect of myelotoxic drugs upon incorporation of $\mathrm{H}^{3}$ thymidine and uridine by bone marrow cells, in vitro, Fed. Proc. 20:71, 1961.

251. Pletscher, A., Besendorf, H., and Bachtold, H. P.: Benzo (a) chinolizine, eine neue Korperklasse mit Wirkung auf den 5-hydroxytryptamine and noradrenalin-Stoffwechsel des gehirns, Arch. exper. Pathol. u. Pharmakol. 232:499, 1958.

252. Pletscher, A., Besendorf, H., and Gey, K. F.: Depression of norepinephrine and 5-hydroxytryptamine in the brain by benzoquinolizine derivatives, Science 129:844, 1959.

253. Pletscher, A., and Gey, K. F.: Interference with the permeation of aromatic monoamines and amino-acids in brain, a possible new type of drug action, Biochem. Pharmacol. 8:82, 1961.

254. Pletscher, A., Shore, P. A., and Brodie, B. B.: Serotonin as a mediator of reserpine action in brain, J. Pharmacol. \& Exper. Therap. 116: 84-89, 1956.

255. Pletscher, A., Shore, P. A., and Brodie, B. B.: Serotonin releasing activity limited to Rauwolfia alkaloids with tranquilizing action, Science 123:992-993, 1956.

256. Plummer, A. J., Sheppard, H., and Schulert, A. R.: The metabolism of reserpine, in Garattini, S., and Ghetti, V., editors: Psychotropic drugs, New York, 1957, Elsevier Publishing Co., pp. 350-362.

257. Posner, H. S.: Metabolism of the phenothiazine tranquilizers in humans, in Abstracts of papers, 136th Meeting, American Chemical Society, Atlantic City, N. J., Sept. 13 to 18, 1959 , p. 81c.

258. Primac, D. W., Mirsky, A. F., and Rosvold, H. E.: Effects of centrally acting drugs on two tests of brain damage, A.M.A. Arch. Neurol. \& Psychiat. 77:328-332, 1957.

259. Quastel, J. H., and Quastel, D. M. J.: The chemistry of brain metabolism in health and disease (American Lecture Series No. 406), Springfield, Ill., 1961, Charles C Thomas, Publisher.

260. Quinn, G. P., Shore, P. A., and Brodie, B. B.: Biochemical and pharmacological studies of Ro 1-9569 (Tetrabenazine), a non-indole tranquilizing agent with reserpine-like effects, J. Pharmacol. \& Exper. Therap. 127:103-109, 1959.

261. Randall, L. O., Atkinson, N., and Iliev, V.: The effect of psychostimulants and psychodepressants on DPN synthesis in the liver,
Arch. internat. pharmacodyn. 129:434-437, 1960.

262. Randall, L. O., Schallek, W., Heise, G. A., Keith, E. F., and Bagdon, R. E.: The psychosedative properties of methaminodiazepoxide, J. Pharmacol. \& Exper. Therap. 129: 163-171, 1960.

263. Read, A. E., Harrison, C. V., and Sherlock, S.: Chronic chlorpromazine jaundice, with particular reference to its relationship to primary biliary cirrhosis, Am. J. Med. 31:249258, 1961.

264. Reckless, D.: Potentiation, Anaesthesia 9:288295, 1954.

265. Rickels, K., Clark, T. W., Ewing, J. H., Klingensmith, W. C., Morris, H. M., and Smock, C. D.: Evaluation of tranquilizing drugs in medical out-patients: Meprobamate, proclorperazine, amobarbital sodium and placebo, J.A.M.A. 171:1649-1656, 1959.

266. Rogers, S. F.: Reserpine and the newborn infant, J.A.M.A. 160:1090, 1956.

267. Rosner, B. S., Ameen, L., and Kolers, P. A.: Effects of Thorazine and Mebaral on alertness in psychotic patients, Current Therap. Res. 1: 55-58, 1959.

268. Ross, S., and Cole, J. O.: Psychopharmacology, Ann. Rev. Psychol. 11:415-438, 1960.

269. Rothstein, C.: An evaluation of the effects of discontinuation of chlorpromazine, New England J. Med. 262:67-69, 1960.

270. Salzman, N. P., and Brodie, B. B.: Physiological disposition and fate of chlorpromazine and a method for its estimation in biological material, J. Pharmacol. \& Exper. Therap. 118: 46-54, 1956.

271. Sanan, S., and Vogt, M.: Effect of drugs on the noradrenaline content of brain and peripheral tissues and its significance, Brit. J. Pharmacol. 18:109-127, 1962.

272. Scherbel, A. L.: Preliminary evaluation of chlordiazepoxide: Double blind study with observations on its effects in various diseases, Am. Pract. \& Digest Treat. 12:275-281, 1961.

273. Schiele, B. C., and Benson, W.: Tranquilizing and related drugs; a guide for the general physician, Postgrad. Med. 23:484-492, 1958.

274. Schlittler, E.: The chemistry of Rauwolfia alkaloids, in Woodson, R. E., Jr., Youngken, H. W., Schlittler, E., and Schneider, J. A., editors: Rauwolfia: Botany, pharmacognosy, chemistry and pharmacology, Boston, 1957, Little, Brown \& Company, pp. 50-108.

275. Schneider, R. A., and Costiloe, J. P.: Effects of centrally acting drugs on conditioning man; the inhibiting and facilitating effects of chlorpromazine, amobarbital and methylphenidyl acetate on the conditioned galvanic skin response, Am. J. M. Sc. 233:418-423, 1957. 
276. Scime, I. A., and Tallant, E. J.: Tetanus-like reactions to proclorperazine (Compazine): Report of eight cases exhibiting extrapyramidal disturbances after small doses, J.A.M.A. 171:1813-1817, 1959.

277. Scott, G. T., and Nading, L. K.: Relative effectiveness of phenothiazine tranquilizing drugs causing release of MSH, Proc. Soc, Exper. Biol. \& Med. 106:88-90, 1961.

278. Seevers, M. H.: The "tranquilizers," Univ. Michigan M. Bull. 23:338-340, 1957.

279. Shagass, C., Azima, H., and Sangowicz, J.: Effect of meprobamate in sustained high dosage on the electroencephalogram and sedation threshold, Electroencephalog. \& Clin. Neurophysiol. 11:275-283, 1959.

280. Shaw, E. B.: Side reactions from tranquilizing drugs, Pediat. Clin. North America 7 : 257-267, 1960.

281. Shaw, E. B., Dermott, R. V., Lee, R., and Burbridge, T. N.: Phenothiazine tranquilizers as a cause of severe seizures, Pediatrics 23:485-492, 1959.

282. Sheatz, G. C.: Studies on chlorpromazine in decerebrate dogs and patients with irreversible brain damage, J. Pharmacol, \& Exper. Therap. 113:47-48, 1955.

283. Sheppard, H., Lucas, R. C., and Tsien, W. H.: The metabolism of reserpine-C $\mathrm{C}^{14}$, Arch. internat. pharmacodyn. 103:256-269, 1955.

284. Sheppard, H., Tsien, W. H., Plummer, A. J., Peets, E. A., Giletti, B. J., and Schulert, A. R.: Brain reserpine levels following large and small doses of reserpine- $\mathrm{H}^{3}$, Proc. Soc. Exper. Biol. \& Med. 97:717-721, 1958.

285. Shideman, F. E., Truax, C. B. M., Parks, R. E., Jr., and Smith, K. U.: Human motion analysis and chlorpromazine, J. Pharmacol. \& Exper. Therap. 122:68A, 1958.

286. Shore, P. A., and Brodie, B. B.: Influence of various drugs on serotonin and norepinephrine in the brain, in Garattini, S., and Ghetti, V., editors: Psychotropic drugs, New York, 1957, Elsevier Publishing Co., pp. 423-427.

287. Smith, K., Ulett, G. A., Johnson, L., and Johnson, M.: Effect of chlorpromazine, reserpine and Frenquel on convulsive threshold, Confinia neurol. 18:26-31, 1958.

288. Spector, S., Kuntzman, R., Shore, P. A., and Brodie, B. B.: Evidence for release of brain amines by reserpine in presence of monoamine oxidase inhibitors: Implication of monoamine oxidase in norepinephrine metabolism in brain, J. Pharmacol. \& Exper. Therap. 130:256-261, 1960.

289. Stewart, W. R. C.: Chlorpromazine jaundice, Am. J. Surg. 100:495-497, 1960.

290. Strickland, K. P., and Noble, D. M.: The in vitro incorporation of inorganic $\mathrm{P}^{32}$ into the phosphatides of different areas of brain. The effect of chlorpromazine administered in vitro and in vivo, in Kety, S. S., and Elkes, J., editors: The regional chemistry, physiology and pharmacology of the nervous system, New York, 1961, Pergamon Press, Inc., pp. 489-496.

291. Su, C., and Lee, C. Y.: The mode of neuromuscular blocking action of chlorpromazine, Brit. J. Pharmacol. 15:88-94, 1960.

292. Sulman, F. G.: The mammotropic effect of ataractic drugs, Biochem. Pharmacol. 8:101$102,1961$.

293. Sulser, F., and Brodie, B. B.: Is reserpine tranquilization linked to change in brain serotonin or brain norepinephrine? Science 131:1440-1441, 1960.

294. Supek, Z., Keckes, S., and Vojvodic, S.: The action of chlorpromazine on water and chloride excretion in rats, Arch. internat. pharmacodyn. 123:253-259, 1960.

295. Supek, Z., Keckes, S., and Vojvodic, S.: The influence of chlorpromazine on the antidiuretic and chloruretic effects of nicotine and posterior pituitary extract, Arch. internat. pharmacodyn. 123:260-263, 1960.

296. Supek, Z., Uroic, B., Gjuris, V., and Keckes, S.: The influence of chlorpromazine on vascular effects of vasopressin and some other pressor agents in dogs, J. Pharm. \& Pharmacol. 11:448, 1959.

297. Sutherland, V. C., Burbridge, T. N., Adams, J. E., and Simon, A.: Cerebral metabolism in problem drinkers under the influence of alcohol and chlorpromazine hydrochloride, J. Appl. Physiol. 15:189-196, 1960.

298. Suzuki, M., Kamio, K., Yasuda, M., Akiyama, S., Mitani, K., Oyama, T., Sato, K., and Yamashita, T.: Effect of chlorpromazine on the function of the endocrine organs, Endocrinol. jap. 3:67-72, 1956.

299. Swain, J. M., and Litteral, E. B.: Prolonged effect of chlorpromazine: EEG findings in a senile group, J. Nerv. \& Ment. Dis. 131:550$553,1960$.

300. Swinyard, E. A., Chin, L., and Fingl, E.: Withdrawal hyperexcitability following chronic administration of meprobamate to mice, Science 125:739-741, 1957.

301. Symposium: Meprobamate and other agents used in mental disturbances, Ann. New York Acad. Sc. 67:671-894, 1957.

302. Symposium on newer antidepressants and other psychotherapeutic drugs: Their use in psychiatry and other branches of medicine, a scientific meeting under the auspices of the University of Texas Medical Branch, Galveston, Texas, November 13-14, 1959. I. Librium (Ro 5-0690), Dis. Nerv. System 21 :suppl.:760,1960 . 
303. Szatmari, A.: Clinical and electroencephalogram investigation on Largactil in psychoses (preliminary study), Am. J. Psychiat. 112: 788-794, 1956.

304. Tatlow, W. F. T., Fisher, C. M., and Dobkin, A. B.: The clinical effects of chlorpromazine on dyskinesia, Canad. M. A. J. 71: 380-381, 1954.

305. Tipton, D. L., Jr., Sutherland, V. C., Burbridge, T. N., and Simon, A.: Effect of chlorpromazine on blood level of alcohol in rabbits, Am. J. Physiol. 200:1007-1010, 1961.

306. Tiwari, N. M., Jindal, M. N., and Jaiswal, C. L.: Effect of tranquilizing agents on water and saline induced diuresis in rats, Arch. internat. pharmacodyn. 128:383-390, 1960.

307. Traugott, N. N., and Balonov, L.: Neirofisiologicheskii analiz aiushchikh pre vvedenii aminazina, Zhur. nevropat i psikhiat. 58: 585-591, 1958. (Abstract in Digest Neurol. \& Psychiat. 26:469, 1958.)

308. Truax, C. B. M.: Effect of chlorpromazine on eyelid conditioning, J. Pharmacol. \& Exper. Therap. 122:77 A, 1958.

309. Tsien, W. H., Sigg, E. B., Sheppard, H., Plummer, A. J., and Schneider, J. A.: Uptake of reserpine- $\mathrm{C}^{14}$ by various areas of the cat brain, Fed. Proc. 15:493, 1956.

310. Tui, C., Riley, E., and Orr, A.: 17-hydrocorticosteroid levels in the peripheral blood of psychotic patients under treatment with chlorpromazine and reserpine: A preliminary study, J. Clin. \& Exper. Psychopath. 17:142. 146, 1956.

311. Tuteur, W., Stiller, R., and Glotzer, J.: Chlorpromazine-Five years later (fifth of a series--a five year study), in Wortes, J., editor: Recent advances in biological psychiatry, New York, 1961, Grune \& Stratton, Inc., vol. 3 , pp. 35-43.

312. Uhr, L., and Miller, J. G.: Experimentally determined effects of emylcamate (Striatran) on performance, autonomic response, and subjective reactions under stress, Am. J. M. Sc. 240:204-212, 1960.

313. U. S. National Institute of Mental Health Psychopharmacology Service Center: Metabolic studies and analytical methods for phenothiazine derivatives used in psychopharmacology, Bethesda, 1961, National Institute of Mental Health.

314. Valcourt, A. J.: Study of excretion of 5-hydroxyindole-acetic acid in mental patients, A.M.A. Arch. Neurol. \& Psychiat. 81:292298, 1958.

315. Vazuka, F. A.: Comparative effects of relaxant drugs on human skeletal muscle hyperactivity, Neurology 8:446-454, 1958.

316. Velardo, J. T.: Induction of pseudopregnancy in adult rats with Trilafon, a highly potent tranquilizer of low toxicity, Fertil. \& Steril. 9: 60-66, 1958.

317. Voelkel, A.: Klinische Wirkung von Pharmaka mit Einfluss auf den Monoaminstoffwechsel des Gehirns, Confinia neurol. 18:144-151, 1958.

318. Waalkes, T. P., Coburn, H., and Terry, L. L.: The effect of reserpine on histamine and serotonin, J. Allergy 30:408-414, 1959.

319. Waalkes, T. P., and Weissbach, H.: In vivo release of histamine from rabbit blood by reserpine, Proc. Soc. Exper. Biol. \& Med. 93: 394-396, 1956.

320. Walkenstein, S. S., Knebel, C. M., MacMullen, J. A., and Seifter, J.: The excretion and distribution of meprobamate and its metabolites, J. Pharmacol. \& Exper. Therap. 123: 254-258, 1958.

321. Walkenstein, S. S., and Seifter, J.: Fate, distribution and excretion of $S^{35}$ promazine, $J$. Pharmacol. \& Exper. Therap. 125:283-286, 1959.

322. Wase, A. W., Christensen, J., and Polley, E.: The accumulation of $S^{35}$-chlorpromazine in brain, A.M.A. Arch. Neurol. \& Psychiat. 75: 54-56, 1956.

323. Watt, D. S., and Crookes, T. G.: The effect of reserpine on perceptual performance in human subjects, in Rothlin, E., editor: Neuropsychopharmacology, Amsterdam, 1961, Elsevier Publishing Company, vol. 2, pp. 410-413.

324. Wechsler, M. B., and Roizin, L.: Tissue levels of chlorpromazine in experimental animals, J. Ment. Sc. 106:1501-1505, 1960.

325. Weiner, N., and Huls, H. N.: Effect of chlorpromazine on levels of adenine nucleotides and creatine phosphate of brain, J. Neurochem. 7:180-185, 1961.

326. White, T.: Histamine metabolism in brain, Lund, 1961, Hakan Ohlssons Boktryckeri.

327. Whitelaw, M. J.: Delay in ovulation and menstruation induced by chlorpromazine, $\mathrm{J}$. Clin. Endocrinol. 16:972, 1956.

328. Whitelaw, M. J.: Chlorpromazine (Thorazine) in the infertile female, Internat. J. Fertil. 5:175-177, 1960.

329. Whitelaw, M. J.: Menstrual irregularities associated with use of methaminodiazepoxide, J.A.M.A. 175:400-401, 1961.

330. Whitten, L. K., and Filmer, D. B.: A photosensitized keratitis in young cattle following the use of phenothiazine as an anthelmintic. I. A clinical description with a note on its widespread occurrence in New Zealand, Australian Vet. J. 23:336-340, 1947.

331. Wilson, C. W. M., and Huby, P. M.: An assessment of the responses to drugs acting on the central nervous system, Clin. Pharmacol. \& Therap. 2:587-598, 1961.

332. Winsor, T.: Human pharmacology of reser- 
pine, Ann. New York Acad. Sc. 59:61-81, 1954.

333. Winsor, T.: Control of conditioned responses of digital blood vessels, Clin. Res. Proc. 5: $66,1957$.

334. Yuwiler, A., and Gerard, R. W.: Urinary chromogens and the Reigelhaupt test for schizophrenia, Fed. Proc. 20:392, 1961.

335. Zbinden, G., Bagdon, R. E., Keith, E. F., Phillips, R. D., and Randall, L. O.: Experimental and clinical toxicology of chlordiazep- oxide (Librium), Toxicol. \& Appl. Pharmacol. 3:619-637, 1961 .

336. Zirkle, G. A., King, P. D., McAtee, O. B., and Van Dyke, R.: Effects of chlorpromazine and alcohol on coordination and judgment, J.A.M.A. 171:1496-1499, 1959.

337. Zirkle, G. A., McAtee, O. B., King, P. D., and Van Dyke, R.: Meprobamate and small amounts of alcohol: Effects on human ability, coordination, and judgment, J.A.M.A. 173: 1823-1825, 1960. 